Discussion Papers of the

Max Planck Institute for

Research on Collective Goods

2020/26

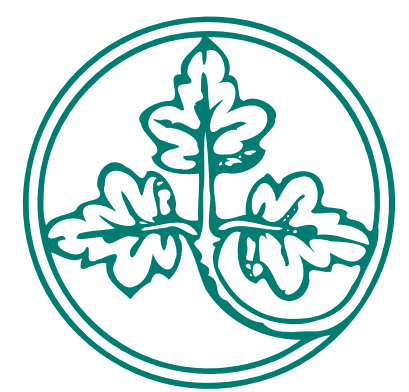

Diagnostic Uncertainty and Insurance Coverage in Credence Goods Markets

Loukas Balafoutas

Helena Fornwagner

Rudolf Kerschbamer

Matthias Sutter

Maryna Tverdostup 


\title{
Diagnostic Uncertainty and Insurance Coverage in Credence Goods Markets
}

\author{
Loukas Balafoutas / Helena Fornwagner / Rudolf Kerschbamer / \\ Matthias Sutter / Maryna Tverdostup
}

November 2020 


\title{
Diagnostic Uncertainty and Insurance Coverage in Credence Goods Markets ${ }^{\S}$
}

\author{
Loukas Balafoutas ${ }^{*}$, Helena Fornwagner ${ }^{\circ}$, Rudolf Kerschbamer, \\ Matthias Sutter ${ }^{*+}$, and Maryna Tverdostup* \\ *University of Innsbruck, Austria, ${ }^{\circ}$ University of Regensburg, Germany \\ \#Max Planck Institute for Research on Collective Goods, IZA Bonn and CESifo Munich, Germany \\ ${ }^{+}$University of Cologne, Germany
}

\begin{abstract}
Credence goods markets - like for health care or repair services - with their informational asymmetries between sellers and customers are prone to fraudulent behavior of sellers and resulting market inefficiencies. We present the first model that considers both diagnostic uncertainty of sellers and the effects of insurance coverage of consumers in a unified framework. We test the model's predictions in a laboratory experiment. Both in theory and in the experiment diagnostic uncertainty decreases the rate of efficient service provision and leads to less trade. In theory, insurance also decreases the rate of efficient service provision, but at the same time it also increases the volume of trade, leading to an ambiguous net effect on welfare. In the experiment, the net effect of insurance coverage on efficiency turns out to be positive. We also uncover an important interaction effect: if consumers are insured, experts invest less in diagnostic precision. We discuss policy implications of our results.
\end{abstract}

JEL-codes: C91, C72, D82, G22

Keywords: Credence goods, diagnostic uncertainty, insurance coverage, welfare, model, experiment

This version: 5 November 2020

\footnotetext{
$\S$ We thank Matthias Heinz, Marco Schwarz, participants at the $93^{\text {rd }}$ Annual Conference of the Western Economic Association, CEAR / MRIC Behavioral Insurance Workshop, $6^{\text {th }}$ Workshop in Behavioral and Experimental Health Economics, Annual Meeting of the Austrian Economic Association, and seminar participants at the University of Düsseldorf (DICE), University of Cologne and MPI Bonn for helpful comments. Financial support from the Austrian Science Fund (FWF) through special research area grant SFB F63 and grant numbers P26901 and P27912, and the Deutsche Forschungsgemeinschaft (DFG, German Research Foundation) under Germany's Excellence Strategy - EXC 2126/1-390838866 is gratefully acknowledged.
} 


\section{Introduction}

In markets for credence goods, expert sellers (e.g., doctors, mechanics, legal or financial advisors) are better informed than consumers about the quality of the good, service, or asset that fits consumers' needs best. This informational advantage of sellers creates incentives to cheat on consumers - by providing too much (overtreatment), too little (undertreatment), or charge for more than has been provided (overcharging). The incentives for fraudulent behavior may cause large inefficiencies. This is particularly worrisome because credence goods markets have a huge volume. In the U.S.A., for instance, health care services alone account for about $17 \%$ of GDP, ${ }^{1}$, the finance industry represents about $20 \%$ of $\mathrm{GDP}^{2}$, and car repair services, another prominent example for credence goods, generated total revenues of about 70 billion Dollars in the U.S.A. in 2019. ${ }^{3}$

In modelling the informational asymmetries between expert sellers and consumers, most of the existing work in the credence goods literature assumes that experts can identify their consumers' needs perfectly, and often at no cost (e.g., Wolinsky, 1993; Dulleck and Kerschbamer, 2006; Dulleck et al., 2011; Hyndman and Ozerturk, 2011; Mimra et al., 2016). In reality, however, this is typically not the case. Diagnosis is usually costly because it requires time and effort, and it may even fail to identify a consumer's problem perfectly, as is nicely illustrated by the field experiment of Schneider (2012) in which about $80 \%$ of car mechanics were not able to identify all three prefabricated defects of a car. Another very common modelling assumption is that consumers bear the full cost of service (Wolinsky, 1993; Dulleck and Kerschbamer, 2006; Fong et al., 2014). In contrast to this assumption, in many real world credence goods markets consumers are insured and thus often have zero, or negligible, marginal costs of additional services. This may induce consumers to think themselves safe with respect to potential problems arising from diagnostic uncertainty. However, insurance may reduce the expert's incentives to invest in costly diagnosis, leading to more diagnostic uncertainty. Conversely, higher uncertainty in the diagnosis is likely to increase consumer expenditures, creating a stronger need for insurance coverage.

In this paper we provide the first unified approach that allows for a systematic theoretical and empirical analysis of the role, and potential interaction, of diagnostic uncertainty and insurance coverage in markets for credence goods. Our model of a credence goods market

\footnotetext{
${ }^{1}$ See http://www.oecd.org/els/health-systems/health-expenditure.htm (accessed 29 October 2020).

${ }^{2}$ See the United States' Bureau of Economic Analysis: https:/www.bea.gov/data/gdp/gdp-industry (accessed 29 October 2020).

3 See https://www.ibisworld.com/industry-trends/market-research-reports/other-services-except-publicadministration/repair-maintenance/auto-mechanics.html (accessed on 29 October 2020).
} 
is based on Dulleck and Kerschbamer (2006), but extends it in several important directions. First, we introduce diagnostic uncertainty. In contrast to the original model's assumption of perfect (and costless) diagnosis, we assume that the expert either receives a signal of exogenous precision about the consumer's problem or has the possibility to reduce the uncertainty about the consumer's needs by exerting costly effort. Second, we introduce insurance coverage into the model, such that the consumer has to pay a premium and in return the service costs are covered by an insurance institution. Third, we allow for malpractice payments for cases where the service fails, which is an important feature of many real world credence goods markets and which has been discussed as an important factor for cost inflation, in particular in the health care sector (Lyu et al., 2017). Fourth, we assume that experts care not only about their own profit, but also about the consumer's material payoff. While the majority of theoretical papers on credence goods assume that experts are rational profit maximizers, there is evidence both from the lab and the field that (at least some) experts have other-regarding preferences (Liu et al., 2013; Brosig-Koch et al., 2016, 2017; Kerschbamer et al., 2017). Our model leads to a series of novel predictions. It predicts that, independently of the price vector for high and low quality service, the average investment in diagnostic precision and the rates of efficient service provision are higher in a regime without insurance coverage. Thus, while insurance is intended to protect consumers, it has an adverse effect on the likelihood of efficient service provision and might therefore be detrimental for consumers and for market efficiency.

Based on the theoretical model, we examine the behavior of experts and consumers on an experimental market for credence goods. The lab experiment involves 576 participants and is based on a 3 x 2 factorial design, varying the following factors: (i) Insurance. Consumers are either fully insured, i.e., the full price of service is covered by an insurance in return for an insurance premium, or not insured, in which case they have to pay the full price of service themselves. (ii) Diagnostic Uncertainty. Experts receive a costly signal about the consumer's problem that - depending on treatment - is either $100 \%$ precise, $70 \%$ precise, or of endogenous precision.

Our experimental results are largely in line with the predictions of the theoretical model. First, we identify diagnostic uncertainty as a major source of inefficiencies in credence goods markets. Comparing the two treatments that vary the exogenously given diagnostic precision, we find that a lower precision leads to lower rates of efficient service provision and lower rates of market entry. To the best of our knowledge, these findings provide the first evidence from an experiment on the possible adverse effects of diagnostic uncertainty in markets for expert services. 
Second, we find strong empirical support for the model's prediction that insurance coverage reduces the rate of efficient service provision. Moreover, our study is the first to identify a hitherto ignored interaction effect. As predicted by our model, insurance leads to lower investments of experts in diagnostic precision in the treatment where precision is endogenous. The net impact of insurance on efficiency is nevertheless positive, significant and quite sizeable, thanks to the counterbalancing force of higher market entry rates from insured consumers.

Third, our theoretical model further predicts that experts' other-regarding preferences affect market outcomes. Based on the experimental elicitation of distributional preferences (Kerschbamer, 2015) one week before the experiment, we find that more prosocial experts are more likely to invest in diagnostic precision, all else equal.

Our paper is related to previous literature in several ways. There is only a small set of papers that deal with diagnostic uncertainty in credence goods markets. Pesendorfer and Wolinsky (2003) present a theoretical model in which experts have to exert costly, but unobservable, effort to identify a consumer's needs. They show that diagnostic precision is negatively affected by price competition on the market. Dulleck and Kerschbamer's (2009) model has a different flavor by letting experts compete against discounters, where the former can exert costly effort to get precise signals about a consumer's needs, while the latter provide no diagnosis, but only sell services. They find that experts are vulnerable to such competition from discounters and may have incentives to undertreat their customers. Bester and Dahm (2018) add subjective evaluation of consumers regarding the success of a service and show in their theoretical model that first-best outcomes can be achieved by separating diagnosis and treatment. Chen et al. (2018) study the design of efficient liability rules in a setting where the expert needs to be provided with proper incentives both in exerting diagnostic effort and in recommending the appropriate treatment. They show that a well-designed liability rule that imposes a penalty on the expert contingent on whether her misbehavior involves over- or undertreatment can achieve the efficient outcome. Liu et al. (2019) consider exogenous heterogeneity in experts' diagnostic abilities. They characterize the equilibria that can arise when abilities are unobservable and show that, in some cases, a higher share of high-ability experts can harm efficiency. Compared to our paper, none of these papers embeds the question of how diagnostic uncertainty affects market outcomes in a framework that considers insurance coverage of consumers, and none also derives predictions that are contingent on the seller's level of prosociality towards the consumer. In fact, the literature on credence goods markets has, so far, dealt with the effects of diagnostic uncertainty and insurance coverage in completely 
separate lines of research, thus ignoring how these two prominent factors on credence goods markets might interact with each other.

Turning to the credence goods literature that investigates the role of insurance, the theoretical model of Sülzle and Wambach (2005) shows that increasing the degree of coinsurance on the consumer side can increase or decrease the likelihood of overcharging on the seller side, depending on the parameters. There are several experimental studies on how insurance coverage affects sellers' and consumers' behavior on credence goods markets. In a laboratory experiment, Huck et al. (2016) find that insurance coverage lets consumers ask for more treatments, and sellers are more likely to overtreat consumers. Several field experiments confirm these patterns. Lu (2014) studies physicians' prescription behavior in a field experiment and reports that doctors write significantly more expensive prescriptions to insured patients. Kerschbamer et al. (2016) find that notebook owners with an insurance are charged about $80 \%$ more than non-insured owners for getting a notebook repaired. None of these papers combines theory and evidence in order to examine and test possible ways in which introducing insurance may affect service provision and market outcomes. Moreover, none interacts insurance with diagnostic uncertainty.

In the following section we introduce the framework of our model. Section 3 presents the model's results. Sections 4 and 5 present the experiment and its results. Section 6 concludes.

\section{Model}

\subsection{The Credence Goods Problem}

We consider an economy populated by ex ante homogeneous consumers and a single expert. Each consumer (he) has either a major problem $\bar{c}$ requiring a high-quality service (HQS) at cost $\bar{c}$, or a minor problem $\underline{c}$ requiring a low-quality service (LQS) at $\operatorname{cost} \underline{c}$, with $\bar{c}>\underline{c}$. For future reference we define $\tilde{c}=\bar{c}-\underline{c}$. The consumer knows that he has an ex ante probability $h$ of having the major problem and a probability of $1-h$ of having the minor one. The consumer derives utility $v>0$ when his problem is solved through a service provided by the expert, and derives zero utility otherwise. While the HQS solves both problems, the LQS solves only the minor problem. The consumer can observe and verify the kind of service he receives ${ }^{4}$, but he only finds out whether the received quality was the needed one when the expert provides LQS for $\bar{c}$ (since in that case his problem remains unsolved). In case a consumer does not receive a service, both the consumer and the expert receive a reservation utility of $o \in[0, v)$.

\footnotetext{
${ }^{4}$ In the jargon of the literature, this means that verifiability applies. This condition rules out fraud in the (over)charging dimension.
} 
Ex ante the expert (she) has the same information as the consumer on the severity of the consumer's problem. In contrast to the consumer, the expert is able to acquire additional information about the consumer's problem by performing a diagnosis. We will consider two cases, one in which the expert is forced to perform a diagnosis and one in which she can freely decide whether to acquire additional information at some cost. We call the former the case with exogenous diagnostic precision (abbreviated as EXO) and the latter the case with endogenous precision (ENDO). For the EXO case we assume that the expert receives a signal $s \in\{\underline{c}, \bar{c}\}$ about the severity of the consumer's problem $\gamma \in\{\underline{c}, \bar{c}\}$ that is correct with probability $\sigma$. We call $\sigma$ the precision level and define it as $\sigma=\operatorname{Pr}(s=\underline{c} \mid \gamma=\underline{c})=\operatorname{Pr}(s=\bar{c} \mid \gamma=\bar{c})$, where $\sigma \in$ $[0.5,1]$, such that $\sigma=0.5$ corresponds to a completely uninformative signal and $\sigma=1$ to a fully precise signal. For the ENDO case, we assume that the expert can freely decide on the precision level $\sigma$ knowing that it is associated with cost $D(\sigma)$, where $D(\sigma)=d(\sigma-0.5)^{2}$ and $d>0$. In line with Inderst and Ottaviani $(2009,2012)$ we assume that the expert faces a penalty $t \in(0, v)$ whenever she prescribes the LQS to a consumer having the major problem. This payment is a compensation for service failure from the expert to the consumer. We assume that an external institution (e.g., a court) verifies that the service failed and then enforces the payment of $t .^{5}$

We denote the exogenously given prices for LQS and HQS by $p$ and $\bar{p}$, and assume $\underline{p}>\underline{c}, \bar{p}>\bar{c}$ and $\bar{p}>\underline{p}$. For future reference, we define the price difference between HQS and LQS as $\tilde{p}=\bar{p}-\underline{p}$ and the price markups for LQS and HQS as $\underline{\Delta}=\underline{p}-\underline{c}$ and $\bar{\Delta}=\bar{p}-\bar{c}$, respectively. Moreover, we distinguish between three types of price vectors: (i) overtreatment (OT) price vectors, where the markup for HQS exceeds the LQS markup $(\bar{\Delta}>\underline{\Delta})$, leading to monetary incentives for the expert to provide HQS; (ii) undertreatment (UT) price vectors, with the LQS markup being higher than the HQS one $(\bar{\Delta}<\underline{\Delta})$ and monetary incentives for providing LQS; and (iii) equal markup (EM) price vectors, with $\bar{\Delta}=\underline{\Delta}$.

Following Liu (2011), Inderst and Ottaviani (2012) and Fong et al. (2014) we allow for the possibility that the expert cares positively about the consumer's well-being. To model this motivation, we introduce the parameter $\lambda \in[0,1]$ and assume that the expert maximizes his own material payoff (weighted by one) plus $\lambda$ times the consumer's surplus. A positive value of $\lambda$ characterizes a prosocial expert, while $\lambda=0$ implies that the expert is completely selfish. ${ }^{6}$

\footnotetext{
${ }^{5}$ This eliminates the possibility of fraudulent behavior of consumers by falsely claiming a failed treatment.

${ }^{6}$ Negative values of $\lambda$ correspond to spiteful experts. Empirically, they are rare $(<2 \%)$ in our experiment.
} 
With respect to insurance, we consider a baseline scenario with no insurance (henceforth $\mathrm{NI}$ ), which means that the entire price for the service is paid by the consumer. The second scheme is one with full insurance (henceforth FI), meaning that an insurance company covers the entire cost of the service. The insurance scheme is exogenously in place. Since we are not interested in the market behavior of the insurance company per se, we introduce the insurer as an implicit institution providing a fair insurance coverage. That is, we assume that the insurance premium (paid by the consumer) is equal to the expected insurance coverage, i.e., the expected surplus of the insurance company is zero.

\subsection{The First-Best}

Before analyzing the actual behavior of the expert, we start by deriving the first-best (FB) outcome, first for the case where the diagnostic precision is exogenously given (EXO-FB) and then for the case where the diagnostic precision is endogenous (ENDO-FB).

EXO-FB: Suppose the consumer is able to observe the diagnosis signal and to implement the service at the same cost as the expert. Which provision strategy would he follow? To address this question we follow a similar line of reasoning as Dulleck and Kerschbamer (2009) and Bester and Dahm (2018). There are three candidates for the efficient solution of the consumer's problem.

Strategy A: Implement the HQS independently of the outcome of the diagnosis.

Strategy B: Implement the LQS independently of the outcome of the diagnosis.

Strategy C: Implement the LQS if the signal suggests that the problem is minor and implement the HQS if the signal suggests that the problem is major.

The efficient strategy is the strategy that minimizes generalized costs defined as the direct costs plus the implied utility loss for the case where the service fails. The generalized cost of Strategy $\mathrm{A}$ is $\bar{c}$, the generalized cost of Strategy B is $\underline{c}+h v$, and the generalized cost of Strategy $\mathrm{C}$ is $(1-h-\sigma+2 h \sigma) \bar{c}+(h+\sigma-2 h \sigma) \underline{c}+h(1-\sigma) v$. For the characterization of the efficient provision policy we need to compare those costs. Along the hyperbola

$$
\sigma_{A C}^{E X O-F B}=\frac{h(v-\tilde{c})}{(1-2 h) \tilde{c}+h v}
$$

strategies $\mathrm{A}$ and $\mathrm{C}$ have the same cost, and along the hyperbola

$$
\sigma_{B C}^{E X O-F B}=\frac{(1-h) \tilde{c}}{(1-2 h) \tilde{c}+h v}
$$

strategies B and C have the same cost. Using these hyperbolas, we can fully characterize the first-best provision strategy. This is done in Proposition 1 (see Appendix A for the proof): 
Proposition 1 (first-best provision strategy with exogenous precision): The first-best provision strategy in EXO is fully characterized in Figure 1. In Area A, efficiency requires to provide the HQS independently of the outcome of the diagnosis (Strategy A); in Area B, efficiency requires to provide the LQS independently of the outcome of the diagnosis (Strategy $B)$; and in Area $C$, efficiency requires to provide the HQS if the outcome of the diagnosis is $\bar{c}$ and the LQS if the outcome is $\underline{\underline{c}}$ (Strategy $C$ ).

Figure 1. Optimal provision strategy with exogenous diagnostic precision

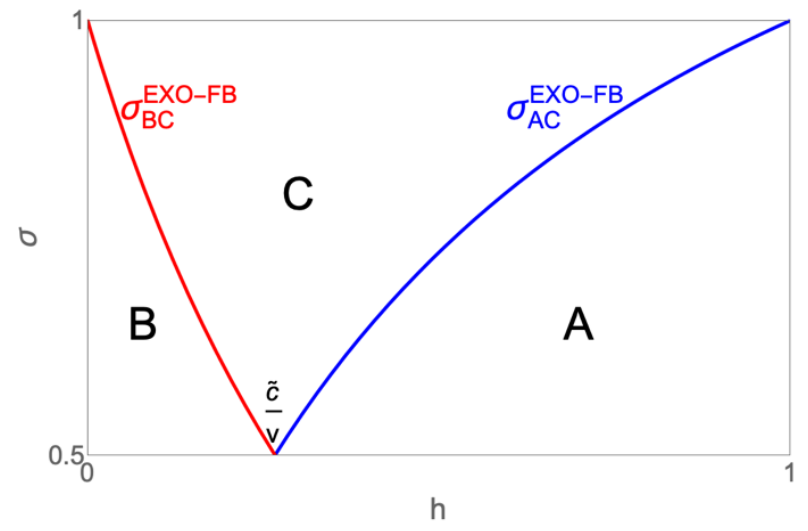

Note: The functions delineating the areas are those defined in equations (1) and (2).

The intuition for the result illustrated in Figure 1 is simple: Strategy $\mathrm{C}$ is optimal if the diagnosis is sufficiently precise and if the likelihood of needing the HQS is neither close to zero nor close to one. If the precision is low, then Strategy A is optimal if the likelihood of needing the HQS is relatively high and Strategy B is optimal if this likelihood is relatively low.

ENDO-FB: Suppose the consumer is able to choose the precision of the signal at the same cost as the expert. Which diagnostic precision would he invest in? To address this question we first solve for the first-best precision level under the assumption that information acquisition (and following the signal) is efficient. This calculation yields

$$
\sigma^{E N D O-F B}=\min \left\{\frac{1}{2}+\frac{(1-h) \tilde{c}+h(v-\tilde{c})}{2 d}, 1\right\},
$$

which implies that $\sigma^{E N D O-F B}=1$ for $d \leq(1-h) \tilde{c}+h(v-\tilde{c})$ and $\sigma^{E N D O-F B}=\frac{1}{2}+$ $\frac{(1-h) \tilde{c}+h(v-\tilde{c})}{2 d}$ for $d>(1-h) \tilde{c}+h(v-\tilde{c})$. Plugging those values into the generalized cost function for Strategy C (as outlined above) and subtracting the associated diagnosis costs yields the generalized cost for the strategy "invest efficiently in diagnosis and follow the signal". Now, investing in diagnosis (and following the signal) is efficient if and only if the generalized cost 
for the latter strategy is lower than the generalized cost associated with Strategy A or Strategy B. Along the hyperbola

$$
\sigma_{A C}^{E N D O-F B}=\frac{1}{2}+\frac{(1-h) \tilde{c}+h(v-\tilde{c}) \pm \sqrt{((1-h) \tilde{c}+h(v-\tilde{c})+d)^{2}-d(d+4 h(v-\tilde{c}))}}{2 d}
$$

strategies $\mathrm{A}$ and $\mathrm{C}$ have the same generalized cost, and along the hyperbola

$$
\sigma_{B C}^{E N D O-F B}=\frac{1}{2}+\frac{(1-h) \tilde{c}+h(v-\tilde{c}) \pm \sqrt{((1-h) \tilde{c}+h(v-\tilde{c})+d)^{2}-d(d+4 \tilde{c}(1-h))}}{2 d}
$$

strategies $\mathrm{B}$ and $\mathrm{C}$ have the same generalized cost. It is easy (although tedious) to show that the optimal diagnostic precision line crosses $\sigma_{A C}^{E N D O-F B}$ and $\sigma_{B C}^{E N D O-F B}$ at the respective vertex. The vertices of $\sigma_{A C}^{E N D O-F B}$ and $\sigma_{B C}^{E N D O-F B}$ are given by

$$
\begin{aligned}
V \sigma_{A C}^{E N D O-F B} & =\frac{\tilde{c}(2 \tilde{c}-v)+d v-\sqrt{d\left(4 \tilde{c}^{2}(3 v-2 \tilde{c})+v^{2}(d-4 \tilde{c})\right)}}{(v-2 \tilde{c})^{2}} \\
V \sigma_{B C}^{E N D O-F B} & =\frac{\tilde{c}(2 \tilde{c}-v)-d v+\sqrt{d\left(4 \tilde{c}^{2}(2 \tilde{c}-3 v)+v^{2}(d+4 \tilde{c})\right)}}{(v-2 \tilde{c})^{2}} .
\end{aligned}
$$

With the help of those expressions we can fully characterize the first-best investment in information acquisition and the first-best provision strategy for the ENDO case. This is done in Proposition 2 (the proof is in Appendix B):

\section{Proposition 2 (first-best investment in information acquisition and first-best provision} strategy with endogenous precision): The first-best investment in information acquisition and the first-best provision strategy in ENDO are fully characterized in Figure 2. In each of the three panels, investing in diagnostic precision and following the signal is efficient for those values of h covered by the black solid line. This black solid line also gives the efficient precision level. For h-values to the left of the (start of the) black solid line, efficiency requires to provide the LQS independently of the outcome of the diagnosis (Strategy B); and for h-values to the right of the (end of the) black solid line, efficiency requires to provide the HQS independently of the outcome of the diagnosis (Strategy A).

The intuition for Proposition 2 is as follows: Obviously, investment in information acquisition is inefficient for parameter constellations in areas A and B of Figure 1 where it is inefficient to follow the signal even when it comes for free. Investment in information acquisition is also inefficient for $h$ values in Area $C$ that are either near the boundary to Area $\mathrm{A}$ or near the boundary to Area B. This follows from the fact that the diagnosis cost increases the generalized cost of Strategy C, while leaving the generalized costs of strategies A and B unaffected. As a consequence, the optimal investment in precision (black solid line in Figure 2, labelled $\sigma^{E N D O-F B}$ ) starts strictly to the right of the red solid line (which is identical to the red 
solid line in Figure 1) and ends strictly to the left of the blue solid line (which is again identical to the corresponding line in Figure 1).

To simplify the exposition, we will from now on focus on the case where $\frac{\tilde{c}}{v}<h$. As can be seen in Figures 1 and 2, under this condition efficiency requires implementing either Strategy A (if $\sigma$ is low) or Strategy C (if $\sigma$ is high), while implementing Strategy B is never efficient.

Figure 2. Optimal provision strategy with choice of diagnostic precision in ENDO

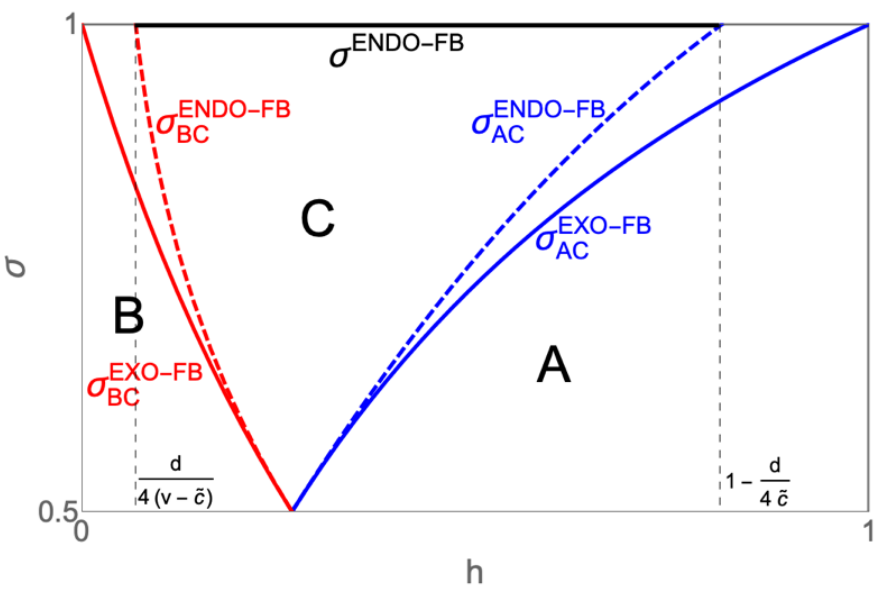

(a) $d \leq \frac{4 \tilde{c}(v-\tilde{c})}{3 v-2 \tilde{c}}$

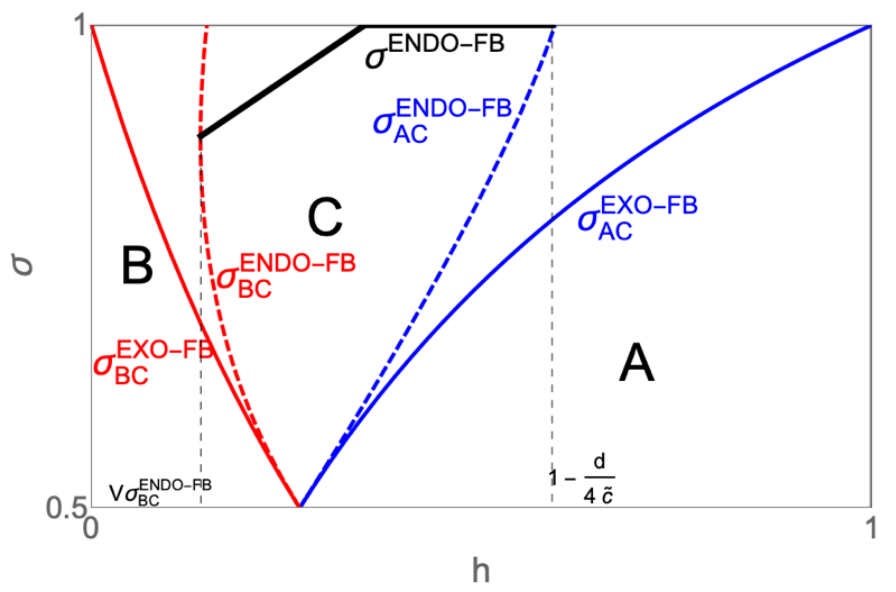

(b) $\frac{4 \tilde{c}(v-\tilde{c})}{3 v-2 \tilde{c}}<d<\frac{4 \tilde{c}(v-\tilde{c})}{v+2 \tilde{c}}$

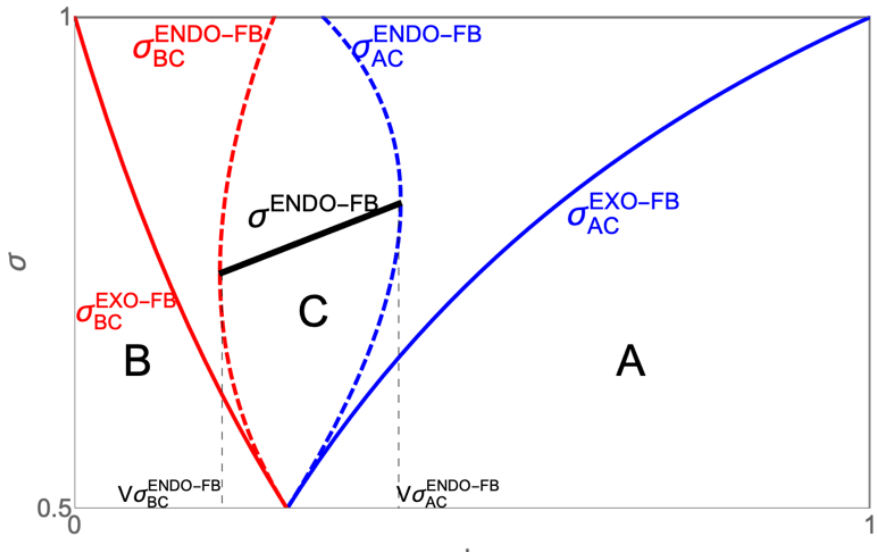

$\mathrm{h}$

$$
\text { (c) } d \geq \frac{4 \tilde{c}(v-\tilde{c})}{v+2 \tilde{c}}
$$

Note: The solid red and the solid blue line are the functions $\sigma_{A C}^{E X O-F B}$ and $\sigma_{B C}^{E X O-F B}$ defined in equations (1) and (2); the dashed red and the dashed blue line are the functions $\sigma_{A C}^{E N D O-F B}$ and $\sigma_{B C}^{E N D O-F B}$ defined in equations (4) and (5); the solid black line is the function $\sigma^{E N D O-F B}$ defined in equation (3); and the dashed vertical lines are the vertices of the functions $\sigma_{A C}^{E N D O-F B}$ and $\sigma_{B C}^{E N D O-F B}$ defined in equations (6) and (7).

\section{Theoretical Results}

We now turn to the actual performance of the market under consideration. We assume that the consumer can neither observe whether the expert has invested in information acquisition (in ENDO), nor which signal she has observed (in EXO and ENDO). 


\subsection{Provision Behavior with Exogenous Diagnostic Precision}

EXO-NI: We first consider the case with exogenous diagnostic precision and no insurance. In this case the expert has the choice between four pure strategies - strategies A, B and C as defined in Subsection 2.2 and Strategy D prescribing to provide the HQS when the signal indicates the minor problem and the LQS when the signal indicates the major problem. In Appendix $\mathrm{C}$ we show that Strategy D is dominated by one of the other three strategies for any given parameter constellation. The other three strategies are associated with the following utilities for the expert:

Strategy A: $\quad \Pi_{A}=\bar{p}-\bar{c}+\lambda[v-\bar{p}]$

Strategy B: $\quad \Pi_{B}=\underline{p}-\underline{c}-h t+\lambda[(1-h) v-\underline{p}+h t]$;

Strategy C: $\quad \Pi_{C}=(1-h-\sigma+2 h \sigma)(\bar{p}-\bar{c})+(h+\sigma-2 h \sigma)(\underline{p}-\underline{c})-h(1-$

$\sigma) t+\lambda[(1-h+h \sigma) v-\underline{p}(h+\sigma-2 h \sigma)-\bar{p}(1-h-\sigma+2 h \sigma)+$ $h(1-\sigma) t]$

For the characterization of the expert's provision policy we need to compare those profits. Along the hyperbola

$$
\sigma_{A C}^{E X O-N I}=f(\lambda, h, \tilde{p}, \tilde{c}, v, t)=\frac{h((1-\lambda) \tilde{p}-\tilde{c})+h \lambda(v-t)+h t}{(1-2 h)(\tilde{c}-(1-\lambda) \tilde{p})+h \lambda(v-t)+h t}
$$

strategies $\mathrm{A}$ and $\mathrm{C}$ yield the same profit, and along the hyperbola

$$
\sigma_{B C}^{E X O-N I}=f(\lambda, h, \tilde{p}, \tilde{c}, v, t)=\frac{(1-h)(\tilde{c}-(1-\lambda) \tilde{p})}{(1-2 h)(\tilde{c}-(1-\lambda) \tilde{p})+h \lambda(v-t)+h t}
$$

strategies B and C yield the same profit. With the help of those hyperbolas we can fully characterize the expert's provision strategy for any constellation of the parameters $v, h$ and $\tilde{c}$, with $v h>\widetilde{c}$, any value of the prosociality parameter $\lambda$, with $\lambda \in[0,1]$, any diagnostic precision $\sigma$, with $\sigma \in[0.5,1]$, any price vector $(\bar{p}, \underline{p})$, with $\tilde{p} \geq 0$, and any transfer $t$, with $t \geq$ 0. This is done in Proposition 3 (see Appendix C for proofs).

Proposition 3 (provision strategy with exogenous precision and no insurance): The expert's actual provision behavior in EXO-NI is fully characterized in Figure 3. In Area A, the expert provides the HQS independently of the outcome of the diagnosis (Strategy A); in Area B, the expert provides the LQS independently of the outcome of the diagnosis (Strategy B); and in Area $C$, the expert provides the HQS if the outcome of the diagnosis is $\bar{c}$ and the LQS if the outcome is $\underline{c}$ (Strategy C). The blue curve in each panel is the hyperbola defined in equation (8), and the red curve in panels (a) and (b) is the hyperbola defined in equation (9). 
Figure 3. Expert provision behavior with exogenous precision and no insurance (EXO-NI)

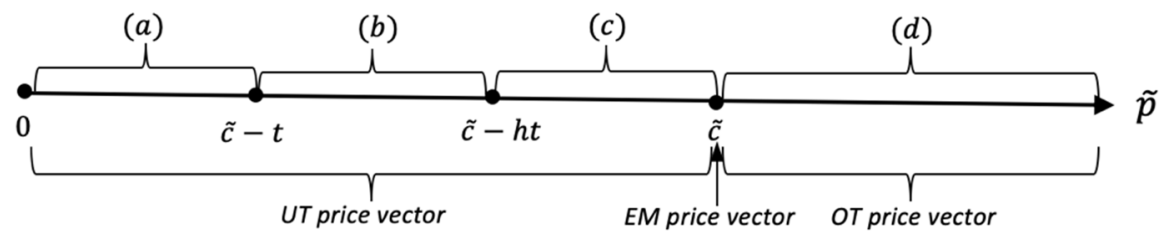

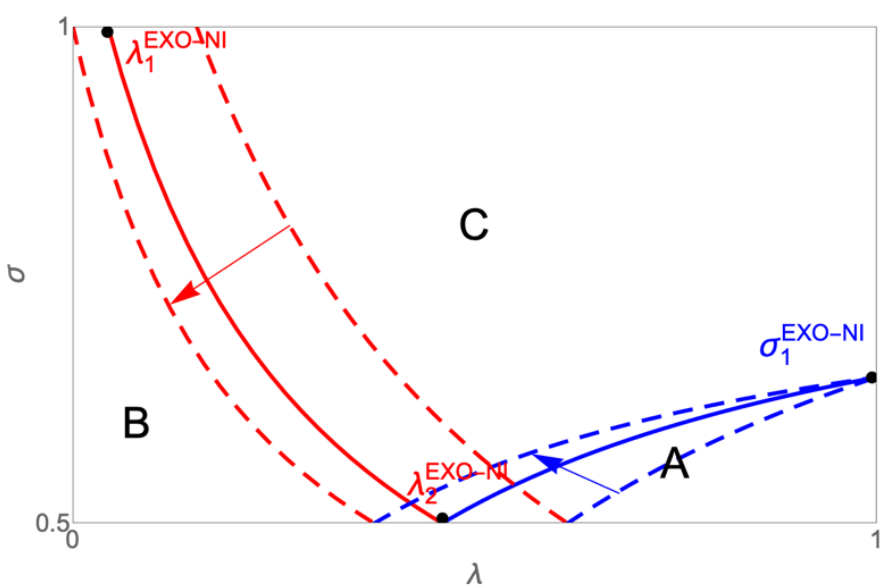

(a) $0<\tilde{p} \leq \tilde{c}-t$

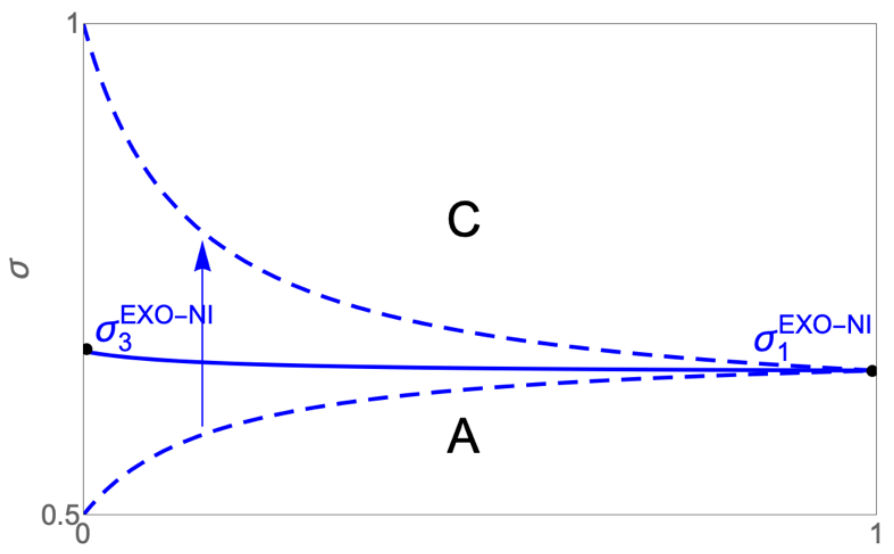

$\lambda$

(c) $\tilde{c}-h t<\tilde{p}<\tilde{c}$

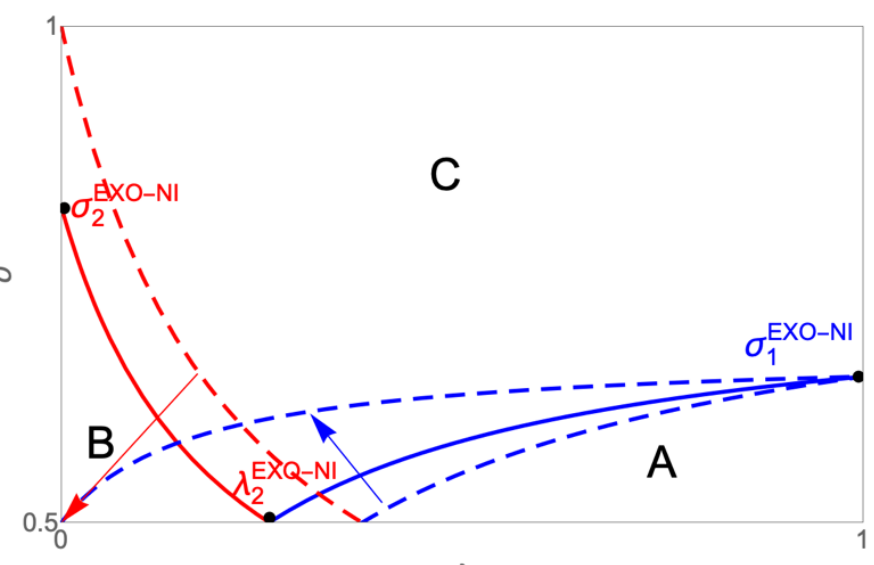

$\lambda$

(b) $\tilde{c}-t<\tilde{p} \leq \tilde{c}-h t$

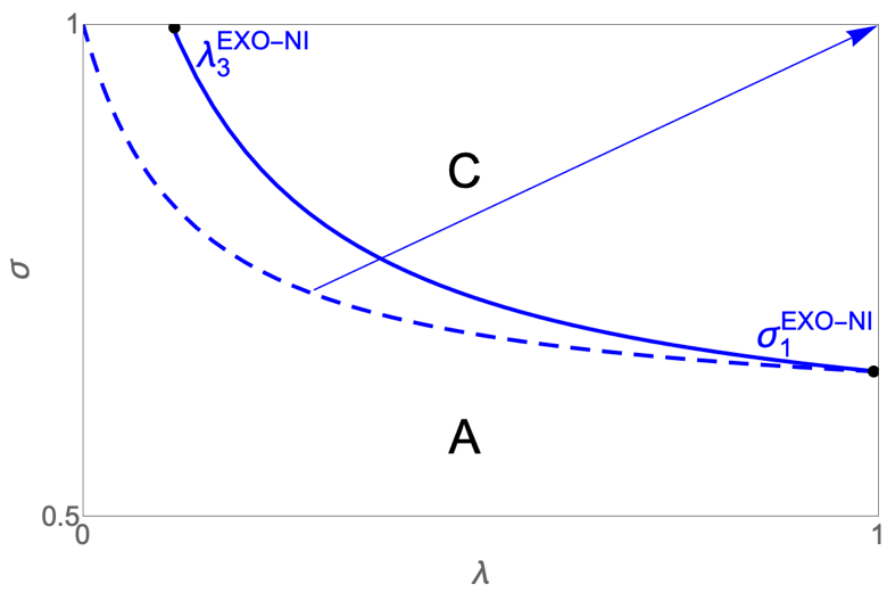

(d) $\tilde{p} \geq \tilde{c}$

Note: The intercept points $\lambda_{1}^{E X O-N I}, \lambda_{2}^{E X O-N I}, \lambda_{3}^{E X O-N I}$ and $\sigma_{1}^{E X O-N I}, \sigma_{2}^{E X O-N I}, \sigma_{3}^{E X O-N I}$ are defined as: $\lambda_{1}^{E X O-N I}=$ $\frac{\tilde{c}-t-\tilde{p}}{v-t-\tilde{p}} ; \quad \lambda_{2}^{E X O-N I}=\frac{\tilde{c}-h t-\tilde{p}}{h(v-t)-\tilde{p}} ; \quad \lambda_{3}^{E X O-N I}=1-\frac{\tilde{c}}{\tilde{p}} ; \sigma_{1}^{E X O-N I}=\frac{1}{2}+\frac{h(v-\tilde{c})-(1-h) \tilde{c}}{2[h(v-\tilde{c})+(1-h) \tilde{c}]} ; \quad \sigma_{2}^{E X O-N I}=\frac{1}{2}+$ $\frac{(1-h)(\tilde{c}-\tilde{p})-h(\tilde{p}-\tilde{c}+t)}{2[h(\tilde{p}-\tilde{c}+t)+(1-h)(\tilde{c}-\tilde{p})]} ; \sigma_{3}^{E X O-N I}=\frac{1}{2}+\frac{h(\tilde{p}-\tilde{c}+t)-(1-h)(\tilde{c}-\tilde{p})}{2[h(\tilde{p}-\tilde{c}+t)+(1-h)(\tilde{c}-\tilde{p})]^{*}}$

A few comments about Figure 3 are in order. Depending on the magnitude of the transfer $t$, one of the following four cases is relevant for the constellation under consideration:

- $t=0$ : For this value of $t$, panels (b) and (c) disappear from Figure 3 and the terms $\lambda_{1}^{E X O-N I}$ and $\lambda_{2}^{E X O-N I}$ in panel (a) converge to 0 if $\tilde{p}$ converges to $\tilde{c}$. If we increase $\tilde{p}$ gradually starting from $\tilde{p}=0$, then we are first (for UT price vectors) in panel (a); if $\tilde{p}$ reaches the critical 
value $\tilde{c}$ (EM price vector) then we are on the boundary between panel (a) and panel (d); and for $\tilde{p}>\tilde{c}$ (OT price vector) we are in the interior of panel (d).

- $t \in(0, \tilde{c})$ : For $t$ in this range, all panels are relevant: If we increase $\tilde{p}$ gradually starting from $\tilde{p}=0$, then we are first in panel (a); if $\tilde{p}$ passes the critical value $\tilde{c}-t$ then we reach panel (b); and so on.

- $t \in\left[\tilde{c}, \frac{\tilde{c}}{h}\right)$ : For $t$ in this range, panel (a) disappears from Figure 3: If we increase $\tilde{p}$ gradually starting from $\tilde{p}=0$, then we are first in panel (b); if $\tilde{p}$ passes the critical value $\tilde{c}-h t$ then we reach panel (c); and so on.

- $t \geq \frac{\tilde{c}}{h}$ : For $t$ in this range, panels (a) and (b) disappear from Figure 3: If we increase $\tilde{p}$ gradually starting from $\tilde{p}=0$, then we are first in panel (c); and if $\tilde{p}$ passes the critical value $\tilde{c}$ then we are in panel (d).

For all values of $t$, and for each panel that is relevant for the $t$ under consideration, we see the effect of increasing $\tilde{p}$ from the lower to the upper boundary of the respective panel by following the respective arrow.

Within each panel, the diagnostic precision $\sigma$ of the constellation gives us a horizontal line (not shown in the figure). If we go to the point $\lambda=1$ on this line, then we see the efficient provision strategy. ${ }^{7}$ The efficient solution depends on $v, \tilde{c}, h$, and $\sigma$, but not on the prices prevalent on the market or the transfer $t$ - that is, the point $\sigma_{1}^{E X O-N I}$ is exactly the same in each of the four panels of Figure 3 and within each panel it changes neither in $\tilde{p}$ nor in $t$. Under our assumption $v h>\tilde{c}$, always providing the HQS is more efficient than always providing the LQS. Thus, efficiency requires to follow the signal (Strategy C) if the signal is precise enough $\left(\sigma>\sigma_{1}^{E X O-N I}\right)$ and to blindly provide HQS (Strategy A) otherwise. ${ }^{8}$ For values of the prosociality parameter $\lambda$ in $[0,1]$ we see which provision strategy the expert of type $\lambda$ actually chooses. As we can see from the figure, there are constellations where efficiency would require to follow the signal, but egoistic and modestly prosocial experts choose Strategy B (panels (a) and (b) for $\sigma>\sigma_{1}^{E X O-N I}$ ) or Strategy A (panels (c) and (d) for $\sigma>\sigma_{1}^{E X O-N I}$ ). However, there are also constellations where efficiency would require to blindly provide the HQS, but egoistic and hardly prosocial experts choose Strategy B while modestly prosocial experts choose Strategy $\mathrm{C}$ (panels (a) and (b) for $\sigma<\sigma_{1}^{E X O-N I}$ ), or where egoistic and scarcely prosocial experts choose Strategy $\mathrm{C}$ (panel (c) for $\sigma_{3}^{E X O-N I}<\sigma<\sigma_{1}^{E X O-N I}$ ).

\footnotetext{
${ }^{7}$ This is due to the fact that an expert with $\lambda=1$ is maximizing the sum of the monetary payoffs of the two agents.

${ }^{8}$ Referring to the $(\sigma, \lambda)$ space, we use the convention that a $\sigma$ with a letter in the subscript denotes a function (of $\lambda$ ) while a $\sigma$ with a number in the subscript denotes a point.
} 
In Figure 3 we can also see that, for any constellation of the parameters $v, h$ and $\tilde{c}$, with $v h>\tilde{c}$, there exist combinations of price vector and transfer that induce all experts to choose the efficient strategy. For $\sigma>\sigma_{1}^{E X O-N I}$, efficiency requires that the expert follows the signal (Strategy C). As we can see in Figure 3, a necessary condition for all experts to choose Strategy $\mathrm{C}$ is that the prices and the transfer are such that the economy is either in panel (b) or in panel (c). ${ }^{9}$ This in turn requires that prices are such that $\tilde{c}>\tilde{p}$ (UT price vector) and that the transfer is strictly positive $(t>0)$. Furthermore, a less precise signal (a lower horizontal line) requires either a larger value of $\tilde{c}-\tilde{p}$, or a larger value of $t$ (because for a less precise signal we need a lower value of $\sigma_{2}^{E X O-N I}$, or $\sigma_{3}^{E X O-N I}$ respectively, to make sure that all experts follow the signal).

Before proceeding, let us shortly discuss how Proposition 3 compares to related findings in the literature. Most of the literature assumes $t=0$ (no transfer), $\sigma=1$ (perfect diagnostic precision) and $\lambda=0$ (completely selfish experts). For this special case, our figure displays the discontinuity in the expert's provision behavior found in large parts of the literature (see Dulleck and Kerschbamer, 2006): For $\tilde{p}<\tilde{c}$ the expert always provides LQS; for $\tilde{p}>\tilde{c}$ she always provides HQS; and at $\tilde{p}=\tilde{c}$ the expert is indifferent between providing LQS and HQS and is therefore assumed to follow the signal. In Figure 3 we see that this insight extends to the case where the diagnosis produces a noisy signal, but only for the special case where $t=0$. With $\sigma<1$ and $t>0$ the equal markup vector (represented by the dashed curve in panel (d)) gives the egoistic expert a strict incentive to provide the HQS independently of the outcome of the diagnosis. This is quite intuitive: With an equal markup vector the expert earns exactly the same immediate profit from selling the LQS and selling the HQS. However, if she sells the LQS in a world where the signal is noisy, then she runs the risk of getting punished (by having to pay $t$ ) while selling the HQS is safe in this regard.

Still staying with the constellation $t=0$ and $\sigma=1$, but allowing now for $\lambda>0$, Figure 3 replicates some of the results in Kerschbamer et al. (2017): If the expert is sufficiently altruistic then she follows the signal even under an UT price vector (panel (a)) or an OT price vector (panel (d)). Our Figure 3 extends the findings from Kerschbamer et al. (2017) by allowing for a compensation for treatment failure $(t>0)$ and for a noisy diagnosis $(\sigma \leq 1)$. As we can see in Figure 3, with $\sigma<1$ the equal markup vector induces experts with a low $\lambda$ to always provide the HQS. We also see that the range of $\lambda$ values for which this is the case becomes larger as the signal becomes less informative.

${ }^{9}$ Only in those panels we can draw a horizontal line above $\sigma_{1}^{E X O-N I}$ such that all points on the line are located in Area C. 
The case where an expert has to pay a transfer $t>0$ as a compensation for service failure has previously been considered by Dulleck and Kerschbamer (2009) for the special case where $\sigma=1$ and $\lambda=0$. The authors show that an expert can be induced to invest in costly diagnosis by a price structure that has $\tilde{p}<\tilde{c}$ and $t>0-$ but not by constellations that have either $\tilde{p} \geq \tilde{c}$ or $t=0$. We can see this in panels (b) and (c) of Figure 3 where the point $\sigma=1$ and $\lambda=0$ lies in the interior of Area $\mathrm{C}-$ which is a necessary condition for an incentive to acquire a costly signal. Our analysis extends that by Dulleck and Kerschbamer (2009) by allowing for arbitrary values of $\lambda$ in $[0,1]$ and arbitrary values of $\sigma$ in $[0.5,1]$.

EXO-FI: We next study the effect of introducing full insurance for consumers on the expert's provision behavior, for the case of exogenous diagnostic precision. Full insurance means that the payment from the consumer to the expert is covered by the insurance company, in exchange for the insurance premium. The latter is paid before the expert-consumer interaction takes place. As a result, the payoff of the consumer is not affected by the price charged by the expert. This changes the expert's payoffs for strategies $\mathrm{A}, \mathrm{B}$ and $\mathrm{C}$ to:

$$
\begin{array}{ll}
\text { Strategy A: } & \Pi_{A}=\bar{p}-\bar{c}-d(\sigma-0.5)^{2}+\lambda v . \\
\text { Strategy B: } & \Pi_{B}=\underline{p}-\underline{c}-h t-d(\sigma-0.5)^{2}+\lambda[(1-h) v+h t] . \\
\text { Strategy C: } & \Pi_{C}=(1-h-\sigma+2 h \sigma)(\bar{p}-\bar{c})+(h+\sigma-2 h \sigma)(\underline{p}-\underline{c})-h(1- \\
& \sigma) t-d(\sigma-0.5)^{2}+\lambda[(1-h+h \sigma)+h(1-\sigma) t] .
\end{array}
$$

Given those payoffs, indifference between strategies $\mathrm{A}$ and $\mathrm{C}$ is reached along the hyperbola

$$
\sigma_{A C}^{E X O-F I}=f(\lambda, h, \tilde{p}, \tilde{c}, v, t)=\frac{h(\tilde{c}-\tilde{p})-h \lambda(v-t)-h t}{(1-2 h)(\tilde{p}-\tilde{c})-h \lambda(v-t)-h t}
$$

and indifference between strategies $\mathrm{B}$ and $\mathrm{C}$ is reached along the hyperbola

$$
\sigma_{B C}^{E X O-F I}=f(\lambda, h, \tilde{p}, \tilde{c}, v, t)=\frac{(1-h)(\tilde{p}-\tilde{c})}{(1-2 h)(\tilde{p}-\tilde{c})-h \lambda(v-t)-h t} .
$$

With the help of those hyperbolas we can fully characterize the expert's provision strategy for the full insurance case. This is done in Proposition 4 (see Appendix D for proofs).

Proposition 4 (provision strategy with exogenous precision and full insurance): The expert's provision behavior in EXO-FI depends on the price markup $\tilde{p}$. For $\tilde{p} \geq \tilde{c}$ all experts opt for Strategy A. For $\tilde{p}<\tilde{c}$, panels (a), (b) and (c) of Figure 4 show the effect of introducing full insurance on the expert's provision behavior. The solid red and the solid blue line as well as the areas $A, B$ and $C$ are defined as in Proposition 3 and they characterize the optimal provision areas in the EXO-NI case. The dashed blue line in panels (a), (b) and (c) is the hyperbola defined in equation (10), and the dashed red line in panels (a) and (b) is the 
hyperbola defined in equation (11). Those curves define the optimal provision areas in the EXO-FI case.

The effect of introducing full insurance coverage depends on the expert's type $\lambda$ and on the prevailing price vector. Selfish experts $(\lambda=0)$ are not affected by the introduction of insurance, since insurance affects the expert's payoff only via the consumer's payoff if $\lambda>0$. For prosocial experts $(\lambda>0)$ the incentive to provide the HQS instead of the LQS is increased by introducing insurance, since the consumer does not directly bear the additional cost of the more expensive service. Which implications does this have for the expert's provision policy? The answer to this question depends on the characteristic of the price vector.

Under EM and OT price vectors the expert faces no trade-off under FI: Her material payoff is maximized by providing the HQS independently of the signal she receives and the same is true for the consumer's payoff. As a consequence, under FI and an EM or an OT price vector, the expert opts for Strategy A under any exogenously given precision level - see panel (d) of Figure 4. Under UT price vectors - covered by panels (a), (b) and (c) of Figure 4 - there is a clear monetary incentive for the expert to provide the LQS, while the consumer's payoff is still maximized with the HQS. As a consequence, a prosocial expert faces a trade-off. If the material incentive to provide the LQS is rather low - as in panel (c) of the figure - the region in which the expert follows the signal (Area C) unambiguously shrinks in favor of the area in which the expert provides the HQS independently of the signal (Area A). More specifically, for all experts with $\lambda>0$ a higher precision level $\sigma$ is needed to choose Strategy $C$ instead of Strategy A, compared to the NI case. If the material incentive to provide the LQS is higher - as in panels (a) and (b) of the figure - the curve separating areas A and C in the FI case is above and to the left of the corresponding line in the NI case, while the curve separating areas B and $\mathrm{C}$ in the FI case is below and to the left of the corresponding line in the NI case. Also, the point $\lambda_{2}^{E X O-F I}$ is located to the left of the point $\lambda_{2}^{E X O-N I}$. This implies that introducing FI expands the range of $\lambda$-types choosing Strategy A for low precision levels to $\left[\lambda_{2}^{E X O-F I}, 1\right]$ and that experts in this range follow Strategy A under a wider range of (low) precision levels as compared to the NI case. At the same time, introducing FI narrows the range of $\lambda$-types choosing Strategy $B$ (for lower precision levels) to $\left[0, \lambda_{2}^{E X O-F I}\right]$ and experts in this range follow Strategy B under a narrower range of (low) precision levels as compared to the NI case.

To sum up, introducing full insurance expands Area A (where the HQS is always provided) at the cost of Area $\mathrm{C}$ (where the expert follows the signal) independently of the prevailing price vector. If the price vector induces strong material incentives to always provide the LQS, then introducing full insurance in addition expands Area $\mathrm{C}$ at the cost of Area B. 
Figure 4. Expert's provision behavior with exogenous precision, no insurance and full insurance (EXO-FI)

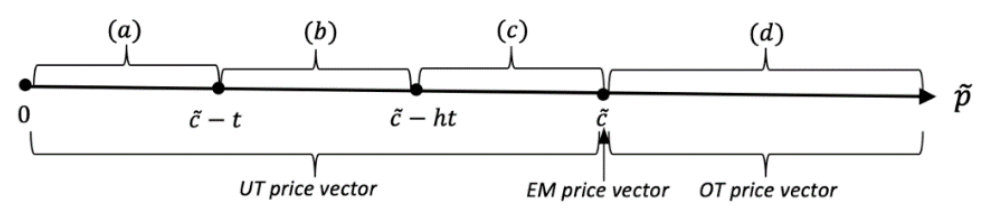

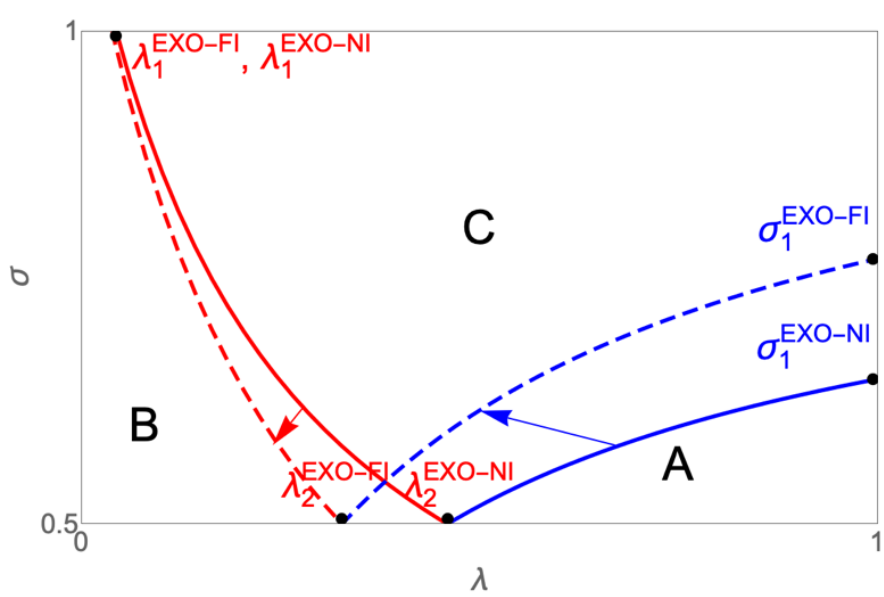

(a) $0<\tilde{p} \leq \tilde{c}-t$

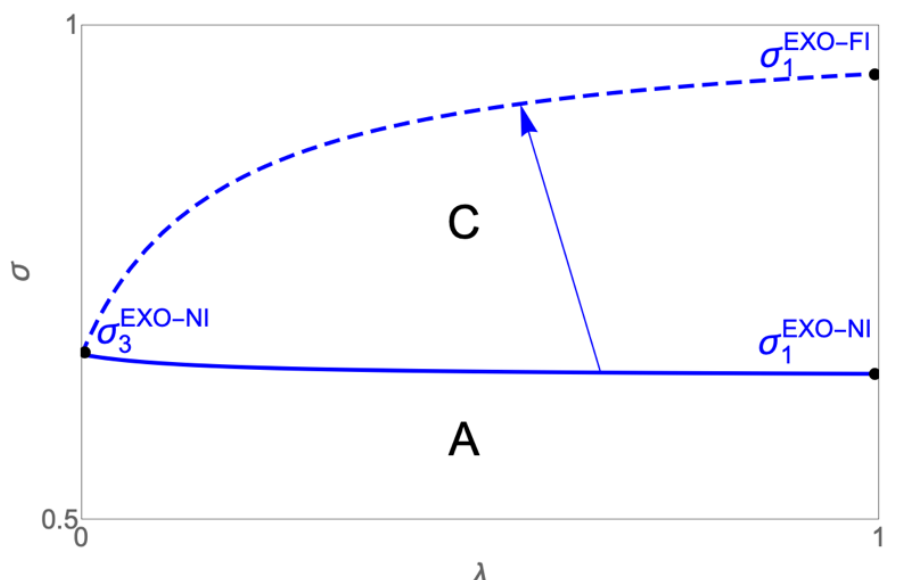

(c) $\tilde{c}-h t<\tilde{p}<\tilde{c}$

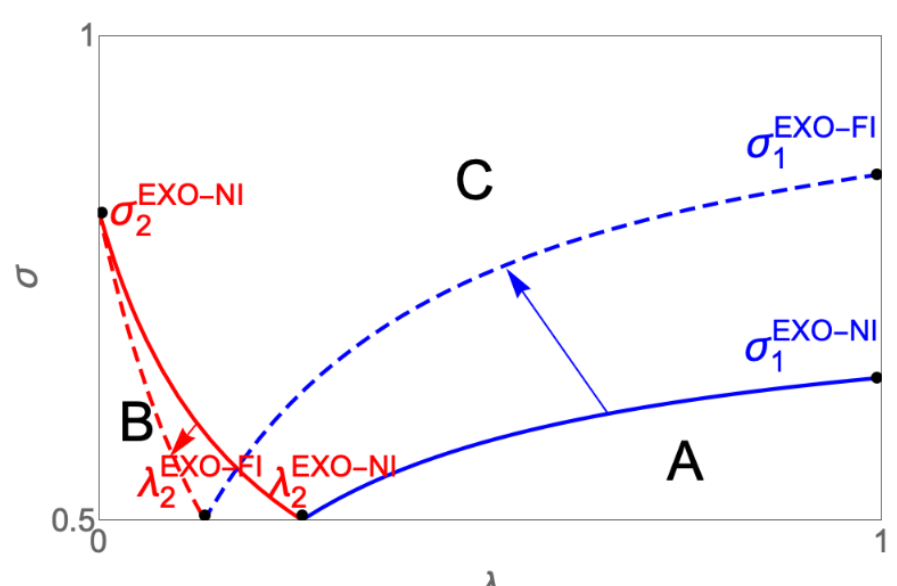

$\lambda$

(b) $\tilde{c}-t<\tilde{p} \leq \tilde{c}-h t$

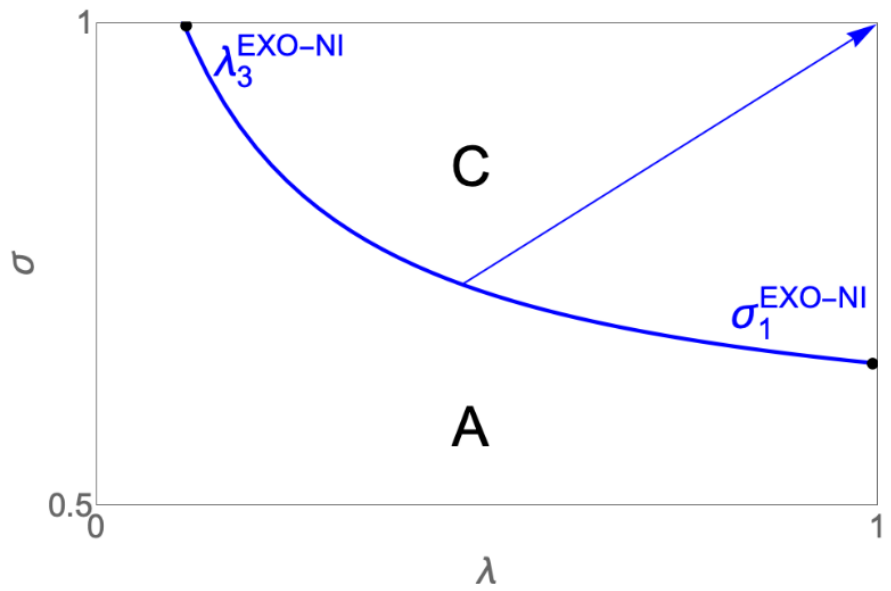

(d) $\tilde{p} \geq \tilde{c}$

Note: This figure shows the effect of introducing insurance in the setting with exogenous precision. The solid red and the solid blue line as well as the areas A, B and C are as defined in Proposition 3 and they characterize the optimal provision areas in the EXO-NI case. The dashed red and the dashed blue line are the hyperbolas defined in equations (10) and (11) and they define the optimal provision areas in the EXO-FI case. The intercept points $\lambda_{1}^{E X O-N I}, \lambda_{2}^{E X O-N I}, \lambda_{3}^{E X O-N I}$ and $\sigma_{1}^{E X O-N I}, \sigma_{2}^{E X O-N I}, \sigma_{3}^{E X O-N I}$ are as defined in the note to Figure 3 . The other intercept points are defined as: $\lambda_{1}^{E X O-F I}=\frac{\tilde{c}-t-\tilde{p}}{v-t} ; \lambda_{2}^{E X O-F I}=\frac{\tilde{c}-h t-\tilde{p}}{h(v-t)} ; ; \sigma_{1}^{E X O-F I}=\frac{1}{2}+\frac{h(v-\tilde{c}+\tilde{p})-(1-h)(\tilde{c}-\tilde{p})}{2[h(v-\tilde{c}+\tilde{p})+(1-h)(\tilde{c}-\tilde{p})]^{*}}$

\subsection{The Expert's Investment in Information Acquisition}

ENDO-NI: We now turn to the expert's payoff-maximizing investment in information acquisition and her payoff-maximizing provision behavior in the ENDO-NI case. To 
characterize them we first solve for the payoff-maximizing precision level under the assumption that information acquisition (and following the signal) is optimal. This calculation yields

$$
\sigma^{E N D O-N I}=\min \left\{\frac{1}{2}+\frac{(1-h)(\tilde{c}-\tilde{p})-h(\tilde{c}-\tilde{p}-t)+\lambda[(1-h) \tilde{p}+h(v-t-\tilde{p})]}{2 d}, 1\right\},
$$

implying that $\sigma^{E N D O-N I} \in(0.5,1)$ for $d>(1-h)(\tilde{c}-\tilde{p})-h(\tilde{c}-\tilde{p}-t)+\lambda[(1-h) \tilde{p}+$ $h(v-t-\tilde{p})]$ and $\sigma^{E N D O-N I}=1$ for $d \leq(1-h)(\tilde{c}-\tilde{p})-h(\tilde{c}-\tilde{p}-t)+\lambda[(1-h) \tilde{p}+$ $h(v-t-\tilde{p})$ ]. Plugging those values into the payoff function for Strategy C (as outlined above) - and taking into account the associated diagnosis cost - yields the payoff for the strategy of investing optimally in diagnosis and following the signal. This strategy is optimal if and only if the payoff from it is larger than the payoffs for Strategy A and Strategy B. The former constraint translates to

$$
\begin{gathered}
\lambda>\lambda_{A C}^{E N D O-N I}=\frac{d(\tilde{p}-h(v-t))+((1-2 h)(\tilde{c}-\tilde{p})+h t)}{((1-2 h) \tilde{p}+h(v-t))}- \\
\frac{\sqrt{d\left[d(\tilde{p}-h(v-t))^{2}+4 h(1-h(((1-2 h) \tilde{p}+h(v-t)(\tilde{p} v-\widetilde{c}(v-t))]\right.}}{((1-2 h) \tilde{p}+h(v-t))^{2}}
\end{gathered}
$$

and the latter constraint translates to

$$
\begin{gathered}
\lambda>\lambda_{B C}^{E N D O-N I}=\frac{d(\tilde{p}-h(v-t))-((1-2 h)(\tilde{c}-\tilde{p})+h t)}{((1-2 h) \tilde{p}+h(v-t))}- \\
\frac{\sqrt{d\left[d(\tilde{p}-h(v-t))^{2}-4 h(1-h)((1-2 h) \tilde{p}+h(v-t)(\tilde{p} v-\widetilde{c}(v-t))]\right.}}{((1-2 h) \tilde{p}+h(v-t))^{2}} .
\end{gathered}
$$

It is rather straightforward to show that $\lambda_{A C}^{E N D O-N I}$ is a monotonically increasing function of $\tilde{p}$, while $\lambda_{B C}^{E N D O-N I}$ is a monotonically decreasing function of $\tilde{p}$. To characterize the payoffmaximizing investment in information acquisition we now ask for which values of $\tilde{p}$ inequality (13) (respectively (14)) is a binding constraint. For

$$
\tilde{p}<\tilde{p}_{A C}=\tilde{c}+\frac{h t}{(1-2 h)}+\frac{d-\sqrt{d(d+4(1-h)(1-2 h) h t)}}{(1-2 h)^{2}}
$$

$\lambda_{A C}^{E N D O-N I}$ is strictly negative, implying that constraint (13) is fulfilled for all experts, while for $\tilde{p}>\tilde{p}_{A C}$ condition (13) is violated for some experts. Also, for

$$
\tilde{p}>\tilde{p}_{B C}=\tilde{c}+\frac{h t}{(1-2 h)}-\frac{d-\sqrt{d(d-4(1-h)(1-2 h) h t)}}{(1-2 h)^{2}}
$$

$\lambda_{B C}^{E N D O-N I}$ is strictly negative, implying that constraint (14) is fulfilled for all experts, while for $\tilde{p}<\tilde{p}_{B C}$ it is violated for some experts. Depending on the value of $d$ we have two constellations to consider. The case when 


$$
\begin{gathered}
d<d^{*}=\frac{\tilde{c}^{2}(1-2 h)^{2}+2 h \tilde{c}(1-2 h)(4(1-h) t+v)-h^{2}\left(4 t^{2}+3 v^{2}+4 h(h-2)(t+v)^{2}\right)}{4(h v-\tilde{c})}+ \\
\frac{(\tilde{c}(2 h-1)+h(v(1-2 h)+2 t(1-h))) \sqrt{4 h(2 h-1)(\tilde{c}(2 h-3)+h t)(v-t)+(\tilde{c}(1-2 h)+h(2 h(t+v)-3 v))^{2}}}{4(h v-\tilde{c})}
\end{gathered}
$$

is characterized in Proposition 5 (the characterization for $d \geq d^{*}$ is very similar and is presented in Appendix E):

\section{Proposition 5 (investment in information acquisition and provision behavior with no}

insurance): For $d<d^{*}$ (as defined in equation (17)) the expert's investment in information acquisition and her provision behavior for the ENDO-NI case are fully characterized in Figure 5. The solid red and the solid blue line as well as the areas $A, B$ and $C$ are defined as in Proposition 3 and they characterize the optimal provision areas in the EXO-NI case. The point $\lambda_{A C}^{E N D O-N I}$ is the critical $\lambda$ defined in equation (13) and the point $\lambda_{B C}^{E N D O-N I}$ is the critical $\lambda$ defined in equation (14) (in both cases, $\lambda_{x C}^{E N D O-N I}=\lambda_{x C \underline{d}}^{E N D O-N I}, x \in\{A, B\}$, for $d \leq(1-h) \tilde{c}+$ $h(v-\tilde{c})$ and $\lambda_{x C}^{E N D O-N I}=\lambda_{x C \bar{d}}^{E N D O-N I}$ for $\left.d>(1-h) \tilde{c}+h(v-\tilde{c})\right)$. With endogenous precision, in constellations falling into panel (a), experts with $\lambda<\lambda_{B C}^{E N D O-N I}$ decide for Strategy $B$ and in constellations falling into panel (c) experts with $\lambda<\lambda_{A C}^{E N D O-N I}$ decide for Strategy A. In all panels, all other experts invest in diagnosis and follow the signal. The optimal precision level for those experts investing in diagnosis is given by equation (12). Increasing the diagnosis cost $d$ decreases the diagnosis effort on the extensive and on the intensive margin: as $d$ increases, fewer experts invest in diagnosis and those who invest, invest in lower precision.

To understand Proposition 5 first note that since the expert's provision depends on the outcome of the diagnosis only under Strategy $\mathrm{C}$, and since investing in diagnosis is costly, the expert will invest zero in information acquisition if she does not plan to rely on the diagnosis outcome even when it comes for free. This implies that in areas A and B of Figure 5 the expert will not invest in information acquisition. For $d=0$, all experts in the interior of Area $\mathrm{C}$ in Figure 5 would choose $\sigma^{E N D O-N I}=1$. However, since $d>0$, some experts will use Strategy $\mathrm{C}$ under EXO-NI, but will not invest in the diagnosis in ENDO-NI - see panels (a) and (c) of Figure 5. In each of the three panels we see which precision level would be efficient by looking at the precision chosen by experts with $\lambda=1$. As we can see from the figure, for low values of $d$ there is a range of (highly prosocial) experts who invest efficiently in information acquisition. This is no longer the case if $d$ exceeds the critical threshold $(1-h) \tilde{c}+$ $h(v-\tilde{c})$. For diagnosis costs that exceed that threshold, all experts (except for those sitting at $\lambda=1$ ) underinvest in diagnostic precision compared to the first-best benchmark. 
Figure 5. Expert's investment in information acquisition and her provision behavior with endogenous precision and no insurance (ENDO-NI)

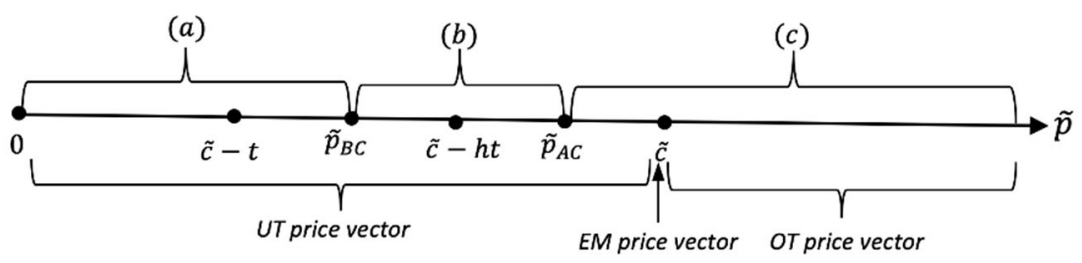

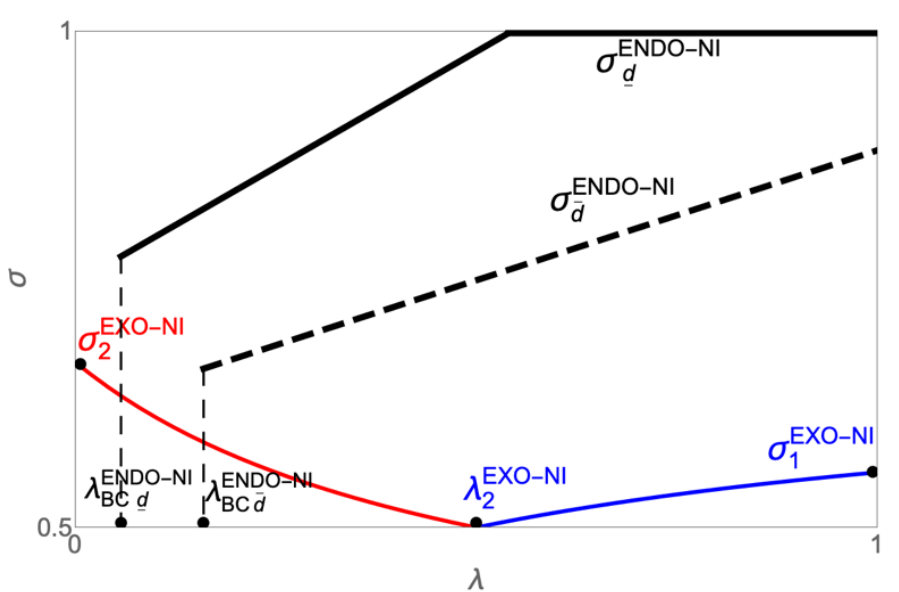

(a) $0<\tilde{p}<\tilde{p}_{B C}$

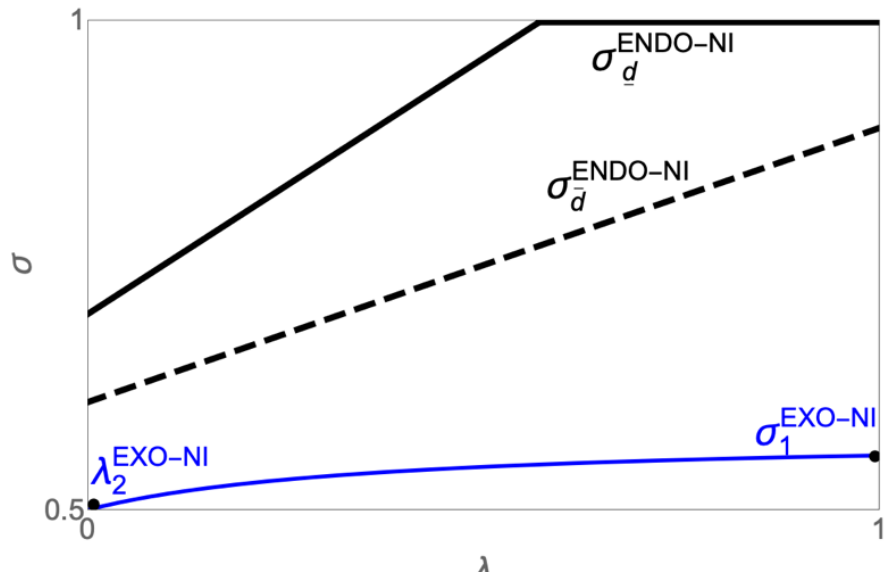

$\lambda$

(b) $\tilde{p}_{B C} \leq \tilde{p} \leq \tilde{p}_{A C}$

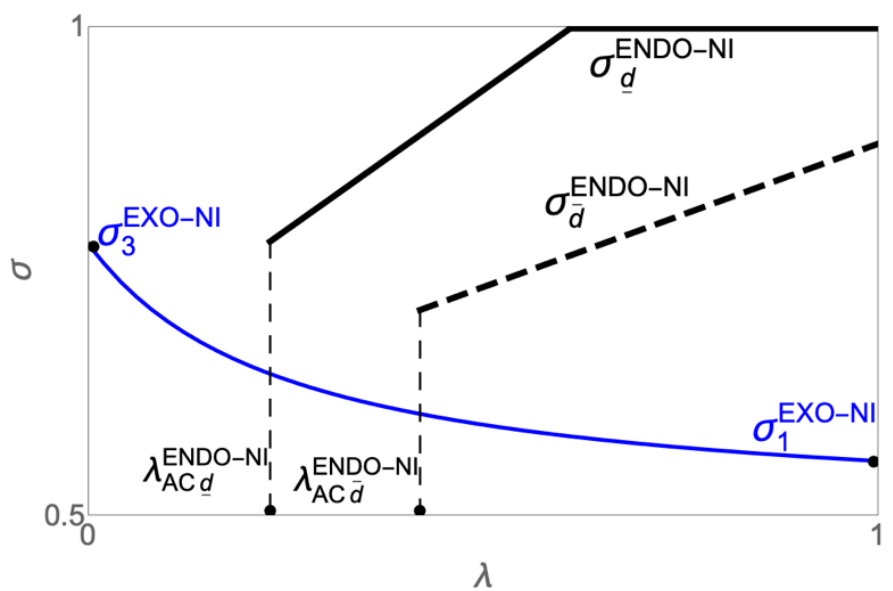

(c) $\tilde{p}>\tilde{p}_{A C}$

ENDO-FI: We now turn to the expert's payoff-maximizing investment in information acquisition and her payoff-maximizing provision behavior in the ENDO-FI case. To characterize those, we proceed as in the ENDO-NI case - but take into account that under FI the consumer does not bear the cost of the service (but pays the insurance premium). The calculation for the payoff-maximizing precision level under the assumption that information acquisition (and following the signal) is optimal now yields

$$
\sigma^{E N D O-F I}=\left\{\frac{1}{2}+\frac{(1-2 h)(\tilde{c}-\tilde{p})+\lambda h(v-t)+h t}{2 d}, 1\right\}
$$


implying that $\sigma^{E N D O-F I} \in(0.5,1)$ for $d>(1-2 h)(\tilde{c}-\tilde{p})+\lambda h(v-t)$ and $\sigma^{E N D O-F I}=1$ for $d \leq(1-2 h)(\tilde{c}-\tilde{p})+\lambda h(v-t)$. Plugging those values into the payoff function for Strategy $\mathrm{C}$ - and taking into account the associated diagnosis cost - yields again the payoff for the strategy "invest optimally in diagnosis and follow the signal". Again, this strategy is optimal if and only if the payoff for it is larger than the payoff associated with strategies A and B. The former constraint translates to

$$
\lambda<\lambda_{A C}^{E N D O-F I}=\frac{(1-h)(\tilde{p}-\tilde{c})+h(\tilde{c}-\tilde{p}-t)+d}{h(v-t)}-\frac{\sqrt{d h^{2}(v-t)^{2}(d+4(h(3 \tilde{c}-\tilde{p})-(\tilde{c}-\tilde{p})))}}{h^{2}(v-t)^{2}}
$$

and the latter constraint translates to

$$
\lambda>\lambda_{B C}^{E N D O-F I}=\frac{(1-h)(\tilde{p}-\tilde{c})+h(\tilde{c}-\tilde{p}-t)-d}{h(v-t)}-\frac{\sqrt{d h^{2}(v-t)^{2}(d+4(1-h)(\tilde{c}-\tilde{p}))}}{h^{2}(v-t)^{2}} .
$$

It is straightforward to show that $\lambda_{A C}^{E N D O-N I}$ and $\lambda_{B C}^{E N D O-N I}$ are both monotonically decreasing in $\tilde{p}$. To characterize the payoff-maximizing investment in information acquisition we now ask for which values of $\tilde{p}$ inequality (19) (respectively (20)) is a binding constraint. For

$$
\tilde{p}<\tilde{p}_{1}=\tilde{c}-\frac{d}{4(1-h)}
$$

$\lambda_{A C}^{E N D O-F I}$ is strictly larger than 1 , implying that constraint (19) is fulfilled by all experts, while for $\tilde{p}>\tilde{p}_{1}$ it is violated for some experts. Also, for $\tilde{p}>\tilde{p}_{A C}$ (as defined in equation (15)) $\lambda_{A C}^{E N D O-F I}$ is strictly negative, implying that constraint (19) is violated for all experts, while for $\tilde{p}<\tilde{p}_{A C}$ it is fulfilled for some experts. Finally, for $\tilde{p}>\tilde{p}_{B C}$ (as defined in equation (16)) $\lambda_{B C}^{E N D O-N I}$ is strictly negative, implying that constraint (20) is fulfilled for all experts, while for $\tilde{p}<\tilde{p}_{B C}$ it is violated for some experts. Depending on the value of $d$ we again have two constellations to consider. For

$$
d<d^{* *}=\frac{4 h t(1-h)}{(6-4 \sqrt{2}) h+4 \sqrt{2}-5}
$$

we have $\tilde{p}_{1}<\tilde{p}_{B C}<\tilde{p}_{A C}$, while for $d>d^{* *}$ we have $\tilde{p}_{B C}<\tilde{p}_{1}<\tilde{p}_{A C}$. With the help of those expressions we can fully characterize the expert's optimal investment in information acquisition and her optimal provision behavior for the ENDO-FI case. For $d<d^{* *}$ this is done in Proposition 6 (the characterization for $d \geq d^{* *}$ is very similar and is presented in Appendix F):

Proposition 6 (investment in information acquisition and provision strategy with endogenous precision and full insurance): The expert's investment in information acquisition and her provision behavior for the ENDO-FI case depends on the price markup $\tilde{p}$. For $\tilde{p} \geq \tilde{p}_{A C}$ (with $\left.\tilde{p}_{A C} \leq \tilde{c}\right)$ no expert invests in information acquisition and all experts decide for Strategy A. For $\tilde{p}<\tilde{p}_{A C}$, panels (a), (b) and (c) of Figure 6 show the effect of introducing full insurance on the expert's investment in information acquisition and her provision behavior for the case 
where $d<d^{* *}$ (as defined in equation (22)). The solid and the dashed black line are as defined in Proposition 5 and they show the optimal precision in the ENDO-NI case. The solid and the dashed red line show the optimal precision in the ENDO-FI case. The point $\lambda_{A C}^{E N D O-F I}$ is the critical $\lambda$ defined in equation (19) and the point $\lambda_{B C}^{E N D O-F I}$ is the critical $\lambda$ defined in equation (20) (in both cases, $\lambda_{x C}^{E N D O-F I}=\lambda_{x C \underline{d}}^{E N D O-F I}$ for $d \leq(1-h) \tilde{c}+h(v-\tilde{c})$ and $\lambda_{x C}^{E N D O-F I}=$ $\lambda_{x C \bar{d}}^{E N D O-F I}$ for $\left.d>(1-h) \tilde{c}+h(v-\tilde{c})\right)$. In constellations falling into panels $(a)$ and $(b)$ experts with $\lambda<\lambda_{B C}^{E N D O-F I}$ decide for Strategy $B$ and in constellations falling into panels (b) and (c) experts with $\lambda>\lambda_{A C}^{E N D O-F I}$ decide for Strategy A. In panels (a), (b) and (c) all other experts invest in diagnosis and follow the signal, while in panel (d) no expert invests in diagnosis. The optimal precision level for those experts investing in diagnosis is given by equation (18). Increasing the diagnosis cost $d$ decreases the diagnosis effort on the extensive and on the intensive margin: As d increases, fewer experts invest in diagnosis and those who invest, invest in lower precision.

Similar to the setting with exogenous precision, the effect of introducing full insurance depends on the expert's type $\lambda$ and on the prevailing price vector. Under EM and OT price vectors the expert faces no trade-off under FI: Her material payoff is maximized by providing the HQS independently of the signal she receives and the same is true for the consumer's payoff. As a consequence, under FI and an EM or an OT price vector the expert decides for Strategy A without investing in information acquisition for any $d \geq 0$. Here, introducing full insurance unambiguously leads to a decrease in the investment in information acquisition, since moderately and highly prosocial experts invest in diagnosis (and follow the signal) in the NI case, while all experts decide for Strategy A without investing in diagnosis in the FI case.

For $t>0$, the same is true for "mild" UT price vectors, where mild means that $\tilde{p}$ lies in the interval $\left[\tilde{p}_{A C}, \widetilde{c}\right]$. If the material incentive to provide the LQS becomes higher, introducing full insurance leads to a decrease in the investment in information acquisition for moderately and highly prosocial agents (in the constellations covered by Figure 6 all experts sitting in the interval $\left[\lambda_{B C}^{E N D O-N I}, 1\right]$ invest weakly less under FI than under NI), but there is also a countervailing effect: Experts in the interval $\left(\lambda_{B C}^{E N D O-F I}, \lambda_{B C}^{E N D O-N I}\right)$ invest in information acquisition under FI but decide for Strategy B under NI. This latter effect is a direct consequence of the fact that for prosocial experts the incentive for choosing Strategy B is decreased by introducing full insurance since they internalize the benefit for the consumer of providing the HQS instead of the LQS, but do not internalize the associated additional cost. 
Figure 6. Expert's provision behavior with endogenous precision, no insurance and full insurance (ENDO-FI)

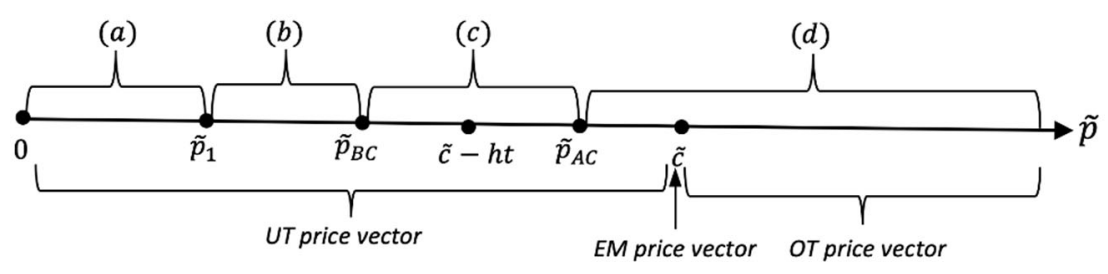

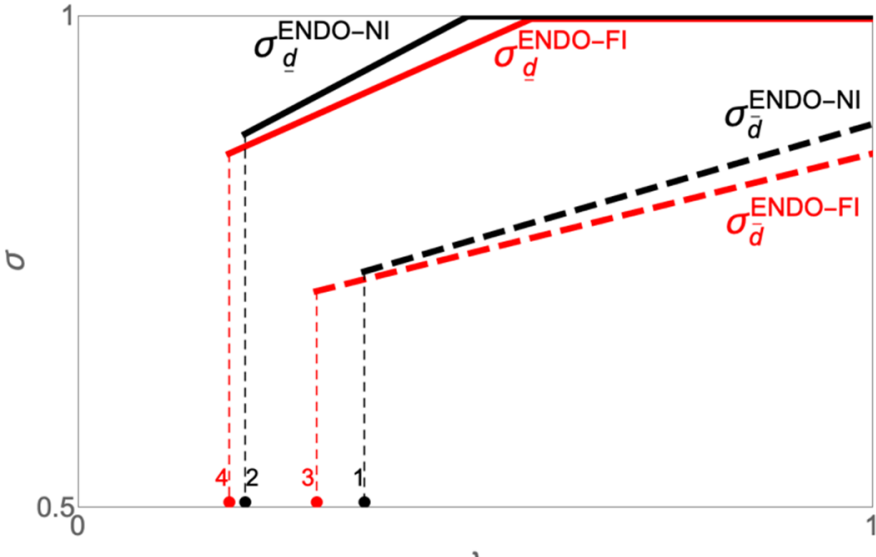

$\lambda$

(a) $0<\tilde{p} \leq \tilde{p}_{1}$

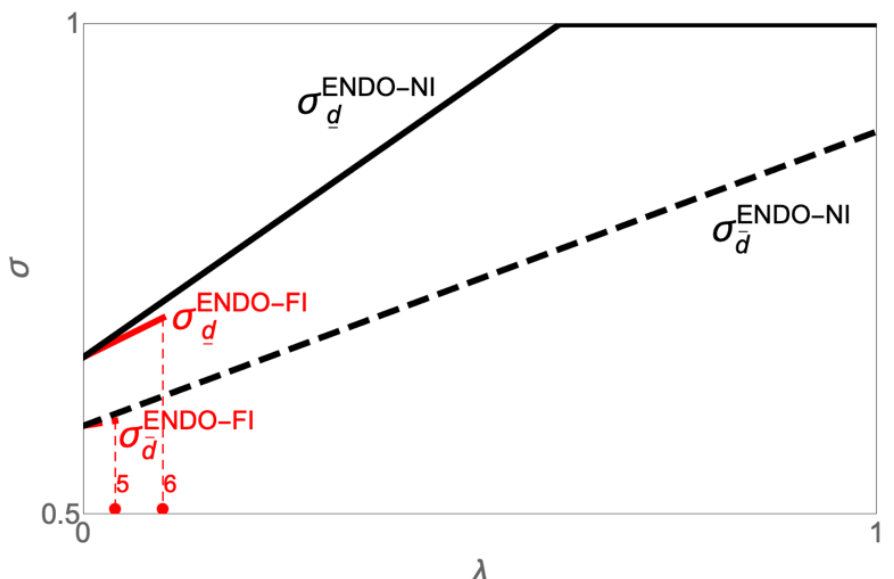

(c) $\tilde{p}_{B C} \leq \tilde{p} \leq \tilde{p}_{A C}$

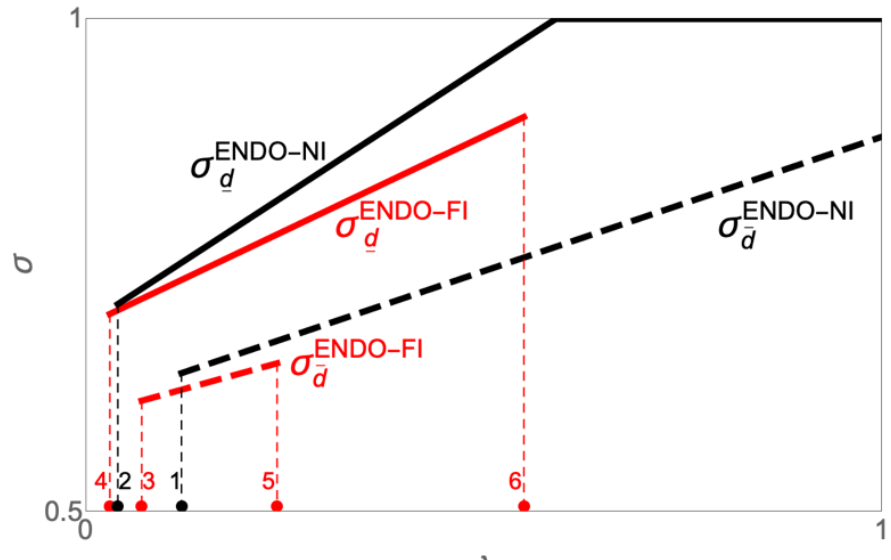

$\lambda$

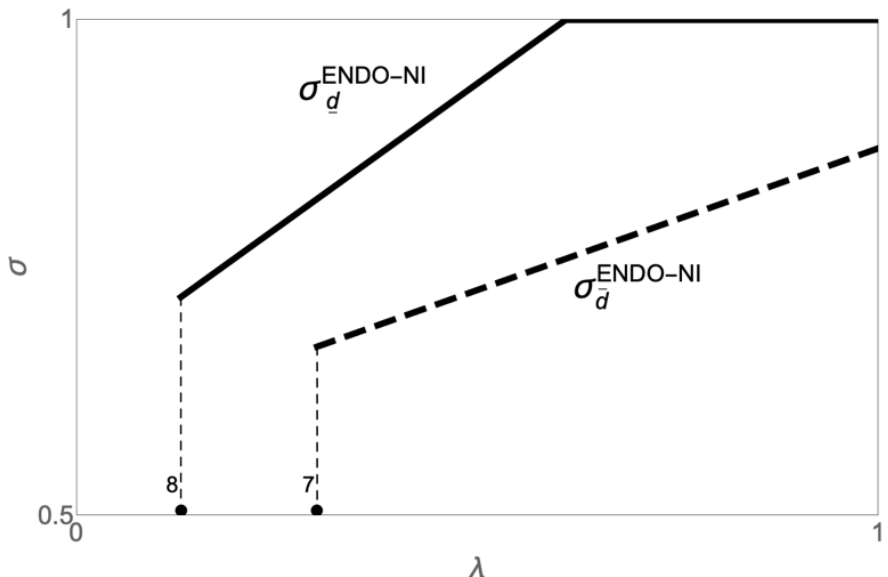

(d) $\tilde{p}>\tilde{p}_{A C}$

Note: The figure shows the effect of introducing insurance on the expert's investment in information acquisition and her provision behavior in the setting with endogenous precision for $d<d^{* *}$ (as defined in equation (22)). The notation on the graph is as follows: Point 1 stands for $\lambda_{B C \bar{d}}^{E N D O-N I}$, point 2 for $\lambda_{B C \underline{d}}^{E N D O-N I}$, point 3 for $\lambda_{B C \bar{d}}^{E N D O-F I}$, point 4 for $\lambda_{B C \underline{d}}^{E N D O-F I}$, point 5 for $\lambda_{A C \bar{d}}^{E N D O-F I}$, point 6 for $\lambda_{A C \underline{d}}^{E N D O-F I}$, point 7 for $\lambda_{A C \bar{d}}^{E N D O-N I}$, and point 8 for $\lambda_{A C \underline{d}}^{E N D O-N I}$.

To sum up, introducing FI unambiguously leads to lower investments in information acquisition under OT, EM and mild UT price vectors. If the price vector induces strong material incentives to provide the LQS independently of the diagnosis outcome - as in panels (a), (b) and (c) of Figure 6 - then introducing FI has an ambiguous effect on the investment in information acquisition: Moderately and highly prosocial experts invest less under FI than 
under NI, but there is also some segment of hardly prosocial experts who decide for Strategy B under NI, but invest in information acquisition under FI.

\subsection{The Consumer's Entry Decision}

For a given institutional environment (EXO-NI, EXO-FI, ENDO-NI or ENDO-FI), given values of the parameters $v, h, \bar{c}, \underline{c}, \sigma, \bar{p}, p$ (which are all common knowledge), given diagnostic precision $\sigma$ (which is common knowledge in the EXO environments) or diagnostic costs $d$ (which are common knowledge in the ENDO environments), the consumer can infer for each $\lambda \in[0,1]$ the expert's investment in diagnostic precision (in the ENDO environments) and her provision behavior (in all environments). He then uses his prior on $\lambda$ to decide whether market entry is profitable. In making this decision he takes into account that under Strategy A he gets a payoff of $v-\bar{p}$, under Strategy B he gets $(1-h) v+h t-\underline{p}$, and under Strategy $\mathrm{C}$ he gets $[1-h(1-\sigma)] v+h(1-\sigma) t-(h+\sigma-2 h \sigma) p-(1-h-\sigma+2 h \sigma) \bar{p}$. Suppose, for instance, we are in the EXO-NI environment, $\sigma$ is equal to 1 , and $\tilde{p}<\tilde{c}-t$. Then the consumer knows that the $\sigma=1$ line of panel (a) of Figure 3 is the relevant provision line for his case. He then uses his prior to assess the mass of experts sitting in the segment $\left[0, \lambda_{1}^{E X O-N I}\right]$, anticipating those experts will implement Strategy B, while all the other experts will decide for Strategy C. Whether or not the consumer will enter the market in this case will depend on his prior and on the price level (i.e., the level of $\bar{p}$ and $\underline{p}$ ) - not only the price difference between HQS and LQS. So, while in theory there is no unambiguous answer for market entry decisions in general, we will use a set of parameters in the experiment that allow for sharp comparative static predictions regarding entry, without making specific assumptions about the consumer's prior.

\section{The Experiment}

\subsection{Experimental Treatments and Parameters}

Our experiment comprises six treatments. In each treatment, we set $h=0.4, v=150, t=50$, and $o=15$. The cost of providing the LQS is $\underline{c}=20$ and the cost for the HQS is $\bar{c}=60$. We fix the price of the LQS across all price vectors at $\underline{p}=60$ and vary the type of the price vector through the price of the HQS. Specifically, for UT we set $\bar{p}=80$; for EM we set $\bar{p}=100$; and for OT we set $\bar{p}=120$. Hence, the three types of price vectors have the following markups of prices over costs: (i) UT: $\underline{\Delta}=40, \bar{\Delta}=20$; (ii) $\mathrm{EM}: \underline{\Delta}=\bar{\Delta}=40$; (iii) $\mathrm{OT}: \underline{\Delta}=40, \bar{\Delta}=60$.

The experimental treatments are presented in Table 1. We implement three conditions with respect to diagnostic precision in a between-subjects design, and two insurance conditions 
in a within-subjects design. Regarding diagnostic precision, in EXO100 the expert receives a fully precise signal about the consumer's problem; in EXO70 the expert receives a signal that is 70\% precise; and in ENDO, the expert can choose between six different precision levels (in $10 \%$ steps from $50 \%$ to $100 \%$ ). The signal is costly in all cases and the cost follows the function $D(\sigma)=40(\sigma-0.5)^{2}$, where $\sigma$ is the precision level. ${ }^{10}$ Hence, the price of diagnostic precision ranges from 0 for $\sigma=0.5$ to 10 for $\sigma=1$. In EXO100 and EXO70, the cost of the corresponding signal is automatically subtracted from the expert's profit. In ENDO, the cost is subtracted only if the expert decides to invest in precision. Otherwise, without any investment, she receives an uninformative, costless signal of $50 \%$ precision.

The second treatment variation refers to insurance: In the three NI (No Insurance) conditions, the price for the service is fully paid by the consumer if he enters the market; in the three FI (Full Insurance) conditions, the price for the service is entirely covered by an insurance institution in return for a premium paid by the consumer. This premium is set to $P=80$, which was calibrated based on preliminary data in order to ensure zero profits for the insurance institution in expectation. Taken together, these variations in insurance and diagnostic precision lead to a $3 \times 2$ factorial design with the six experimental treatments shown in Table 1 .

Table 1: Treatment variation

\begin{tabular}{lcc}
\hline \hline & No Insurance & Full Insurance \\
\hline Exogenously precise signal $(\sigma=1)$ & EXO100-NI & EXO100-FI \\
\hline Exogenously imprecise signal $(\sigma=0.7)$ & EXO70-NI & EXO70-FI \\
\hline Endogenous choice of precision $(\sigma \in[0.5,1])$ & ENDO-NI & ENDO-FI \\
\hline
\end{tabular}

\subsection{Procedure}

We conducted our controlled laboratory experiment in the Innsbruck EconLab during 2018 with students enrolled at the University of Innsbruck. All experimental sessions were computerized with oTree (Chen et al., 2016) and we recruited subjects via H-ROOT (Bock et al., 2014). A total of 576 students participated in the experiment. ${ }^{11}$ The number of participants in a session was either 16 or 24 and we used matching groups of eight subjects each, of which four were in the role of experts and four in the role of consumers. Table 2 presents the number of participants,

\footnotetext{
${ }^{10}$ In the theoretical model of sections 2 and 3 the expert receives the diagnosis for free in the EXO environment. Assuming instead that the signal is costly in all cases does not change any of the derivations. We decided to make the signal costly in the experiment in order to keep the EXO and ENDO treatments more comparable.

${ }^{11}$ Every subject participated in one session only and no subject had ever participated in a credence goods experiment before.
} 
sessions, and matching groups per treatment. The total number of observations follows from each subject making decisions over 24 periods.

Table 2: Observations

\begin{tabular}{ccccc}
\hline \hline Treatment & Subjects & Observations & Sessions & Matching Groups \\
\hline EXO100 & 200 & 4,800 & 9 & 25 \\
EXO70 & 192 & 4,608 & 8 & 24 \\
ENDO & 184 & 4,416 & 8 & 23 \\
\hline Total & 576 & 13,824 & 26 & 72 \\
\hline
\end{tabular}

\subsubsection{Online Part (Elicitation of prosocial preferences)}

After subjects registered for an experimental session, they received a separate mail with a link for an online part of the experiment that was run before the lab session. In the online part we elicited social preferences by means of the Equality Equivalence Test, henceforth EET (Kerschbamer, 2015). The EET exposes subjects to two choice lists, one located in the domain of advantageous inequality and the other located in the domain of disadvantageous inequality. The switching points of a subject in the two lists are then used to infer the prosociality (the $\lambda$ parameter) of the subject separately for each domain. For the domain of disadvantageous inequality we denote the inferred $\lambda$ as $\lambda_{D}$ and for the domain of advantageous inequality we denote it as $\lambda_{A}$. The parameters of the EET and the details of the calculation of $\lambda$ are provided in Appendix G. Decisions were incentivized and participants were paid after the lab part had also been concluded. ${ }^{12}$

\subsubsection{Lab Part (The market experiment)}

Table 3 summarizes the experimental design for the credence goods market experiment. Treatments EXO100, EXO70, and ENDO were implemented between subjects, while the two insurance conditions (NI vs. FI) were varied within subjects such that a group played 12 periods with insurance and 12 periods without, in blocks of six periods and with balanced ordering. Hence, within each of the three diagnostic precision treatments, we vary two insurance schemes (NI, FI), three types of price vectors (UT, OT, and EM), and the type of the consumer's problem (minor or major). This results in 12 possible combinations of insurance, price vector, and consumer's problem. We implemented each of these combinations twice within one session, yielding a total of 24 periods to be played. We find no order effects with regards to the sequence of the different combinations, therefore we pool all orders in the analysis.

\footnotetext{
${ }^{12}$ See https://osf.io/9kwja/?view only=fc4ec287dd4144d79bee2d688add23ab for the Experimental Instructions.
} 
Table 3: Experimental design

\begin{tabular}{|c|c|c|c|}
\hline Treatments & $\begin{array}{c}\text { Diagnostic } \\
\text { precision } \\
\text { (between subjects) }\end{array}$ & $\begin{array}{c}\text { Insurance } \\
\text { (within subjects) }\end{array}$ & $\begin{array}{c}\text { Price vectors } \\
\text { (within } \\
\text { subjects) }\end{array}$ \\
\hline EXO100 & $100 \%$ & $\begin{array}{l}12 \text { periods for each of EXO100- } \\
\text { FI \& EXO100-NI* }\end{array}$ & $\begin{array}{l}8 \text { periods for } \\
\text { each of UT, } \\
\text { EM, OT }\end{array}$ \\
\hline EXO70 & $70 \%$ & $\begin{array}{l}12 \text { periods for each of EXO70-FI } \\
\text { \& EXO70-NI* }\end{array}$ & $\begin{array}{l}8 \text { periods for } \\
\text { each of UT, } \\
\text { EM, OT }\end{array}$ \\
\hline ENDO & $\begin{array}{c}\text { expert chooses } \\
\text { among }\{50 \%, 60 \% \text {, } \\
70 \%, 80 \%, 90 \%, \\
100 \%\}\end{array}$ & $\begin{array}{l}12 \text { periods for each of ENDO-FI } \\
\text { \& ENDO-NI* }\end{array}$ & $\begin{array}{l}8 \text { periods for } \\
\text { each of UT, } \\
\text { EM, OT }\end{array}$ \\
\hline
\end{tabular}

Given that the stage game is repeated for 24 periods, the matching protocol of participants is important. We used matching groups of eight participants, with four experts and four consumers (in fixed roles). Consumers and experts were randomly re-matched within a matching group after each period. We use matching groups as independent observations in our non-parametric tests and cluster for matching groups in our regressions.

All parameters, the underlying treatment condition, as well as the matching procedure were made common knowledge to participants by reading them out aloud at the beginning of each session. The average session duration was 1.5 hours and all payoffs were stated in ECU (experimental currency units). Participants received 80 ECU as starting endowment, and earned on average a total of $1,466.98 \mathrm{ECU}$ (exchange rate $80 \mathrm{ECU}=1 \mathrm{Euro}$ ) from the online part and all 24 periods in the lab.

The timing of a one-period interaction was as follows: At the beginning of each period, both players were informed about the prevailing price vector (UT, EM, or OT) and the insurance condition (NI or FI). Each period consisted of three different stages. In Stage 1, the consumer decided whether or not to enter the market. At the same time the expert received a signal on the consumer's problem in EXO100 and EXO70, while in ENDO she decided how much to invest in diagnostic precision and afterwards received the signal with the chosen precision. In Stage 2 , the consumer was inactive while the expert decided on the quality of the service using the strategy method (i.e., this decision was implemented if the consumer had entered the market). In Stage 3, both players were informed about their own payoff. ${ }^{13}$ From the second period onwards, experts and consumers could see the history of their own decisions and payoffs from

\footnotetext{
${ }^{13}$ The consumer is not informed about the quality that was actually needed, but in case of undertreatment he can infer from the payoff that insufficient quality was provided.
} 
previous periods, but had no information about their previous interaction partners. After the 24 periods of the market game, we elicited participants' self-reported risk attitudes, based on a simple question that asked them to report their risk tolerance on a scale from 0 ("completely risk averse") to 10 (“completely risk-seeking”). Moreover, we collected data on their gender, age, study program, and highest educational degree.

\subsection{Predictions}

In this section we derive the predictions for the experiment. In the derivations we assume that there is a positive mass of experts of each type $\lambda$ in $[0,1]$ and that this is correctly anticipated by consumers.

\subsubsection{Predictions for the Provision Strategy in the EXO Treatments}

Figure 7 characterizes the predicted and the efficient service provision policy for the EXO treatments of the experiment. The solid lines (blue for the UT price vector, red for the EM price vector and orange for the OT price vector) define the provision areas for the NI case: The expert decides for Strategy A below the respective line and for Strategy $\mathrm{C}$ above the line. In the FI case, all experts decide for Strategy A under the EM and the OT price vector while for the UT price vector the dashed blue line defines the provision areas (Strategy A below the line and Strategy $\mathrm{C}$ above the line). By looking at the provision behavior of the $\lambda=1$ expert in the NI case we see what efficiency would prescribe for the parameters of the experiment: For both values of the diagnostic precision and independently of whether insurance is present or absent efficiency requires following Strategy C.

From Figure 7 we see that, independently of the level of diagnostic precision and of whether insurance is present or absent, a weakly larger range of $\lambda$ values is in Area $C$ under the UT than under the EM price vector, and a weakly larger range of $\lambda$ values is in Area $\mathrm{C}$ under the EM than under the OT price vector. ${ }^{14}$ We therefore conclude:

Prediction 1 (impact of price vector on provision strategy in EXO): Independently of the level of diagnostic precision and of whether insurance is present or absent, experts decide for the efficient service provision policy (of following the signal) more frequently under the UT than under the EM price vector, and more frequently under the EM than under the OT price vector.

\footnotetext{
${ }^{14}$ Under NI the relation between UT and EM is strict for $\sigma=0.7$ (while all experts are predicted to choose Strategy $\mathrm{C}$ under both price vectors for $\sigma=1$ ), and the relation between EM and OT is strict for both precision levels. Under FI the relation between UT and EM is strict for both precision levels while the relation between EM and OT is strict for none.
} 
Figure 7. Expert's provision in EXO treatments

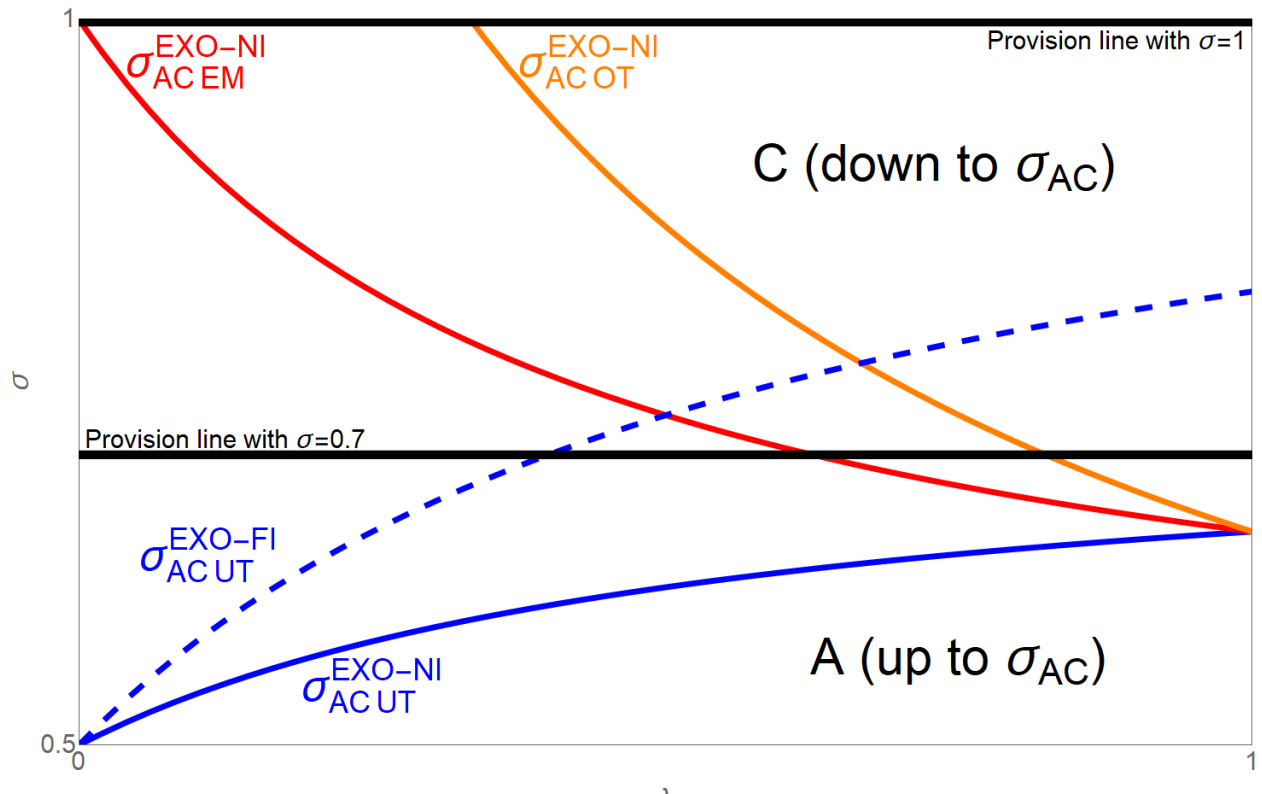

Note: The figure characterizes the predicted provision behavior for the EXO treatments of the experiment. The two black solid horizontal lines (at $\sigma=1$ and at $\sigma=0.7$ ) are the provision lines for the two precision levels implemented in EXO.

Turning to the impact of diagnostic precision on the expert's provision strategy we see that, for each price vector and for both insurance regimes, a larger range of $\lambda$ values is in Area $C$ on the upper $(\sigma=1)$ line than on the lower $(\sigma=0.7)$ line. ${ }^{15}$ This leads to the following prediction:

Prediction 2 (impact of diagnostic precision on provision strategy in EXO): Independently of the price vector and of whether insurance is present or absent, experts decide for the efficient service provision policy more frequently under $\sigma=1$ than under $\sigma=0.7$.

Next we compare - for each price vector and for each provision line - the provision strategy under NI with the one under FI. We see that, in all comparisons, a strictly larger range of $\lambda$ values is in the $\mathrm{C}$-area under NI than under FI. This leads to the third prediction:

Prediction 3 (impact of insurance on provision strategy in EXO): Independently of the price vector and the level of diagnostic precision, experts decide for the efficient service provision policy more frequently under NI than under FI.

A final interesting comparison for the EXO case regards the impact of the prosociality parameter $\lambda$ on the expert's provision strategy. Looking at Figure 7 we see that, under NI, more

\footnotetext{
${ }^{15}$ Under NI the relation is strict for the EM and for the OT price vector, while in FI it is strict only for the UT price vector.
} 
prosocial experts are weakly more inclined to choose Strategy $C$ than less prosocial ones. Under FI, however, the relationship is reversed. ${ }^{16} \mathrm{We}$ thus conclude:

Prediction 4 (impact of prosocial motivation on provision strategy in EXO): Independently of the price vector and of the level of diagnostic precision, under NI more prosocial experts decide for the efficient service provision policy more frequently than less prosocial ones, while under FI the relationship is reversed.

\subsubsection{Predictions for the Investment in Information Acquisition in ENDO}

Figure 8 characterizes the expert's investment in information acquisition and her provision behavior for the ENDO treatments. From this figure we see that efficiency prescribes to acquire a fully precise signal $(\sigma=1)$. We also see that in both insurance regimes and for all $\lambda \in[0,1]$, the investment in information acquisition is weakly higher under the UT price vector than under the EM price vector and weakly higher under the EM than under the OT price vector. ${ }^{17}$ This leads to the following prediction:

\section{Prediction 5 (impact of price vector on investment in diagnostic precision in ENDO):}

Independently of whether insurance is present or absent, the average investment in diagnostic precision is higher (and thereby closer to the efficient level) under the UT price vector than under the EM price vector, and higher under the EM price vector than under the OT price vector.

Comparing the two insurance regimes we see that, for all price vectors and all $\lambda \in[0,1]$ the investment in information acquisition is weakly higher under NI than under FI. ${ }^{18}$ This leads to the next prediction:

Prediction 6 (impact of insurance on investment in diagnostic precision in ENDO): Independently of the price vector, the average investment in diagnostic precision is higher (and thereby closer to the efficient level) in NI than in FI.

Our last prediction for the expert's behavior in the ENDO treatments regards the impact of the prosociality parameter $\lambda$ on the expert's investment in information acquisition. Looking at

\footnotetext{
${ }^{16}$ Under NI the relationship is strict for the OT price vector under $\sigma=1$ and for the OT and the EM price vector under $\sigma=0.7$, while under FI the (reversed) relationship is strict for the UT price vector under $\sigma=0.7$.

${ }^{17}$ Under NI the relation between the UT and the EM price vector is strict for all $\lambda \leq 0.42$ and the relation between the EM and the OT price vector is strict for all $\lambda \in(0.42,0.61)$. Under FI the relation between the UT and the EM price vector is strict for all $\lambda \in[0,1]$, while the relationship between the EM and the OT price vector is strict for none.

${ }^{18}$ Under the UT price vector the relation is strict for all $\lambda<0.4$, under the EM price vector it is strict for all $\lambda \geq$ 0.42 , and under the OT price vector it is strict for all $\lambda \geq 0.61$.
} 
Figure 8 we see that, independently of the price vector and the insurance regime, investment in information acquisition is higher for more prosocial experts than for less prosocial ones. ${ }^{19} \mathrm{We}$ conclude:

\section{Prediction 7 (impact of prosocial motivation on investment in diagnostic precision in} ENDO): Independently of the price vector and of insurance being present or absent, the average investment in diagnostic precision is higher (and thereby closer to the efficient level) for more prosocial experts than for less prosocial ones.

Figure 8. Expert's investment in information acquisition in ENDO

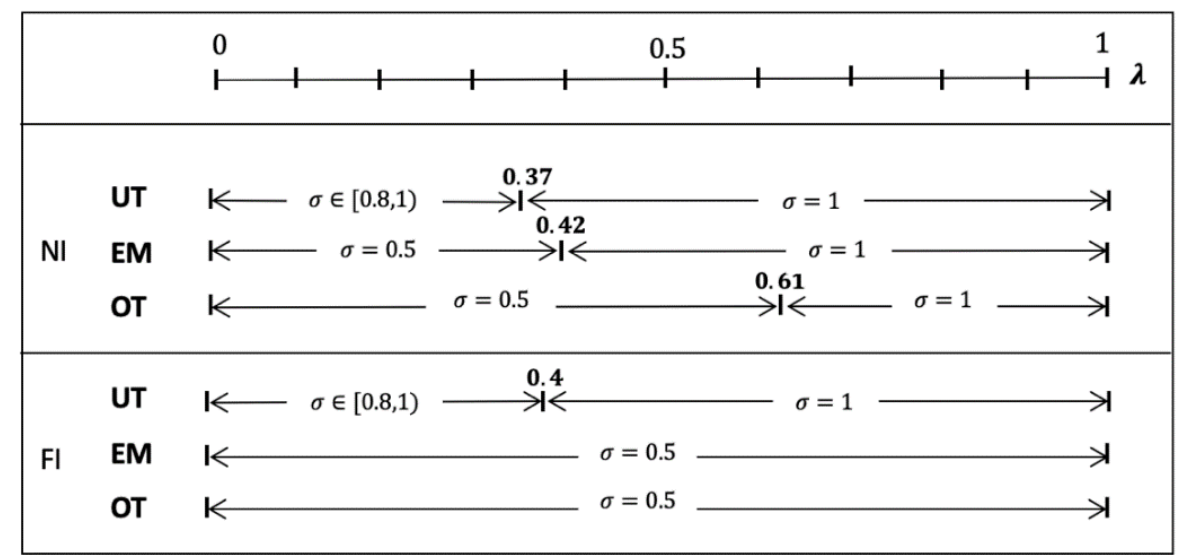

Note: The figure characterizes the expert's investment in information acquisition and her provision behavior for the ENDO treatments. For each insurance regime (NI vs FI) and each price vector (UT, EM, or OT) the figure characterizes the chosen precision level for each $\lambda$ in $[0,1]$. In constellations where $\sigma>0.5$ the expert invests in diagnostic precision and then follows the signal. In constellations where $\sigma=0.5$ the expert abstains from investing in diagnostic precision and chooses Strategy A.

\subsubsection{Predictions for Consumer Behavior in EXO and ENDO}

Next, we turn to consumers' market entry decisions. First, note that for the chosen parameters the consumer's payoff under Strategy A is $v-\bar{p}=150-\bar{p}$ for both diagnostic precision levels, while his payoff under Strategy $\mathrm{C}$ depends on the diagnostic precision: For $\sigma=1$ the consumer's payoff under Strategy C is $v-(1-h) \underline{p}-h \bar{p}=150-0.4 \underline{p}-0.6 \bar{p}$, while for $\sigma=0.7 \quad$ it $\quad$ is $\quad[1-h(1-\sigma)] v+h(1-\sigma) t-(h+\sigma-2 h \sigma) \underline{p}-(1-h-\sigma+2 h \sigma) \bar{p}=$ $138-0.54 \underline{p}-0.46 \bar{p}$. Thus, since $\bar{p}>\underline{p}$ for any given price vector, the consumer unambiguously prefers Strategy C over Strategy A if the signal is perfect; however, under $\sigma=$ 0.7 the consumer prefers Strategy C over Strategy A only if $0.54(\bar{p}-\underline{p}) \geq 12$. Given our

\footnotetext{
${ }^{19}$ Under NI, the relationship is strict for all price vectors while under FI the relationship is strict only for the UT price vector.
} 
parametrization this latter inequality is satisfied for the EM and the OT price vector, but not for the UT vector. What can we conclude from this?

First consider the case where $\sigma=1$. For this case we get a clear prediction: As we move from the UT to the EM and then to the OT price vector, the range of $\lambda$ values for which Strategy $\mathrm{C}$ is provided decreases and at the same time $\bar{p}$ increases. This is unambiguously bad news for the consumer. However, when moving from the UT to the EM price vector for $\sigma=0.7$, we have two opposing effects. On the one hand, the range of $\lambda$ values for which Strategy $\mathrm{C}$ is implemented decreases, which is now good news for the consumer. On the other hand, the price $\bar{p}$ increases, which is again bad news for the consumer. Which of the two price vectors is preferred by the consumer depends on a comparison between his payoff from Strategy $\mathrm{C}$ under the UT price vector and his payoff from Strategy A under the EM price vector. This comparison shows that the consumer strictly prefers Strategy C under the UT price vector over Strategy A under the EM price vector, since $138-[0.54] \cdot 60-[0.46] \cdot 80>150-100$.

Using similar arguments for the impact of insurance and for the impact of diagnostic precision and also considering the case where the diagnostic precision is endogenous we arrive at the following prediction:

\section{Prediction 8 (factors affecting consumer's entry decision in EXO and ENDO):}

(a) Both in EXO and in ENDO, consumers are more likely to enter the market under the UT than under the EM price vector, and more likely under the EM than the OT price vector. In both settings this prediction holds independently of whether insurance is in place, and in EXO it also holds independently of the level of diagnostic precision.

(b) Both in EXO and in ENDO, consumers are more likely to enter the market under FI than under NI. In both settings this prediction holds independently of the price vector, and in EXO it also holds independently of the level of diagnostic precision.

(c) In the EXO case, consumers have a more pronounced tendency to enter the market if the diagnostic precision is $100 \%$ rather than $70 \%$. This prediction holds independently of the price vector and of whether insurance is in place.

\section{Results of the experiment}

Table 4 provides summary statistics for participants in the different treatments and overall. Importantly, randomization across the three treatments was successful in every dimension $(p>$ 0.05 for all variables in Table $4 ; \chi^{2}$ tests and Kruskal-Wallis tests). 
Table 4: Summary statistics

\begin{tabular}{lcccccccc}
\hline \hline & All & $\mathbf{N}$ & EXO100 & $\mathbf{N}$ & EXO70 & $\mathbf{N}$ & ENDO & $\mathbf{N}$ \\
\hline Age & 22.66 & 576 & 22.47 & 200 & 22.86 & 192 & 22.66 & 184 \\
& $(3.51)$ & & $(3.26)$ & & $(4.00)$ & & $(3.22)$ & \\
$\%$ Female & 51.56 & 576 & 56.00 & 200 & 48.96 & 192 & 49.46 & 184 \\
& & & & & & & & \\
$\lambda_{D}$ & 0.10 & 247 & 0.08 & 86 & 0.05 & 83 & 0.17 & 78 \\
& $(0.36)$ & & $(0.37)$ & & $(0.38)$ & & $(0.29)$ & \\
$\lambda_{A}$ & 0.39 & 247 & 0.41 & 86 & 0.42 & 83 & 0.33 & 78 \\
& $(0.36)$ & & $(0.39)$ & & $(0.37)$ & & $(0.31)$ & \\
Risk Measure & 5.59 & 528 & 5.33 & 176 & 5.72 & 192 & 5.72 & 160 \\
& $(2.11)$ & & $(2.10)$ & & $(2.04)$ & & $(2.20)$ & \\
Aver. payment & 14.41 & 288 & 13.94 & 100 & 14.74 & 96 & 14.57 & 92 \\
experts in $€$ & $(1.39)$ & & $(1.29)$ & & $(1.25)$ & & $(1.51)$ & \\
Aver. payment & 22.26 & 288 & 23.78 & 100 & 20.70 & 96 & 22.24 & 92 \\
consumers in $€$ & $(2.35)$ & & $(1.83)$ & & $(1.92)$ & & $(2.19)$ & \\
\hline
\end{tabular}

Notes: Means of all dependent variables, except Female, which refers to the percentage of female subjects. Risk Measure is constructed from responses to a survey question on a scale from 0 "completely risk averse" to 10 "fully risk seeking" and is available for participants in all but one session. $\lambda_{D}$ and $\lambda_{A}$ are elicited in the EET and range from $-5 / 6$ to $5 / 6$, with higher values indicating stronger prosociality. These variables are reported for experts and are missing for 41 subjects, for whom choices in the online part of the experiment could not be matched to the lab part. Standard deviations in parentheses.

\subsection{Impact of Prices, Diagnostic Uncertainty and Insurance on Provision Policy (EXO)}

Here we test predictions 1-3 for the EXO treatments. The main variable of interest is the rate of efficient service provision: This is the frequency (or, in the regressions, the likelihood) with which experts decide for the efficient provision policy by following the signal they received.

Prediction 1 relates efficient service provision to prices. In line with this prediction, the share of experts who follow the signal (pooling the two exogenous treatments and the two insurance conditions) is highest with the UT price vector $(84.1 \%)$, intermediate with the EM price vector $(72.5 \%)$, and lowest with the OT price vector $(64.4 \%)$. The difference to the EM vector is significant both for the UT and the OT vector $\left(p<0.01, \chi^{2}\right.$-tests) ${ }^{20}$

Figure 9 shows the rate of efficient service provision broken down by diagnostic precision and insurance. The treatment differences support predictions 2 and 3 . In both insurance regimes, efficient service provision is more frequent in EXO100 than in EXO70 (pooled rates $76.8 \%$ vs. $70.4 \%, p<0.01, \chi^{2-t e s t) . ~ T h i s ~ r e s u l t ~ c o n f i r m s ~ t h e ~ n e g a t i v e ~ i m p a c t ~ o f ~}$

\footnotetext{
${ }^{20}$ All non-parametric statistical tests reported in the paper treat one matching group (of eight subjects) as one independent observation, resulting in conservative estimates of statistical significance.
} 
diagnostic uncertainty on the rate at which expert sellers follow the signal that they receive. Turning to Prediction 3, the data confirm that the rate of efficient service provision declines when insurance is in place (FI) compared to when this is not the case (NI), both in EXO100 and EXO70 (pooled rates $71.5 \%$ vs. $75.9 \%, p=0.037, \chi^{2}$ test).

Figure 9: Rate of efficient service provision, by treatment

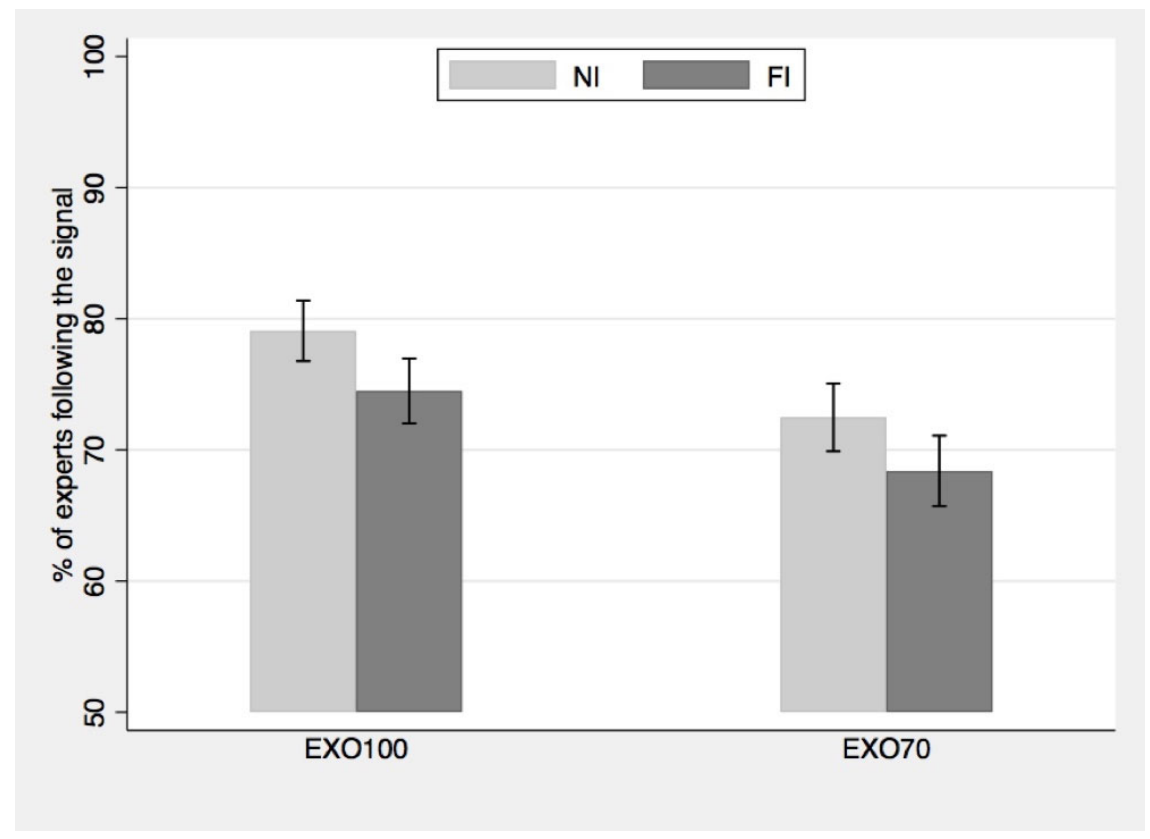

Note: All bars in the figure include $95 \%$ confidence intervals.

We also test Predictions 1-3 in the multivariate Probit regressions shown in Table 5. The dependent variable in the regressions is whether the expert followed the signal. We first provide one parsimonious specification with only price vector, insurance, and treatment dummies as right hand side variables. We then add a specification that further includes the interaction term between insurance and treatment, as well as a third one that includes additional control variables (period, experts' demographics, risk attitudes, and the two social preference parameters $\lambda_{\mathrm{D}}$ and $\lambda_{\mathrm{A}}$ ). All regressions in this section include subject random effects and report standard errors clustered at the matching group level. In all Probit specifications, we report marginal effects of the coefficients.

The regression results confirm predictions 1-3. The negative effects of diagnostic uncertainty and insurance coverage on efficient service provision are captured by the statistically and economically significantly coefficients for EXO70 and Insurance. Diagnostic uncertainty decreases the likelihood of efficient service provision by up to 8 percentage points (about $10 \%$ of the overall average) and insurance decreases it by up to 5 percentage points (about $6 \%$ of the average). These are average effects for each dimension, while the interaction 
between the two dimensions is insignificant in (2). The regressions also confirm the strong positive (negative) effect of UT (OT) price vector on efficient service provision compared to the omitted category of EM prices, with effect sizes being about 8 percentage points for the OT vector and larger than 10 percentage points for the UT vector. All control variables are insignificant, with the exception of Period, suggesting a slight downward time trend. To test Prediction 4, we have run the column (3) regressions for NI and FI separately. ${ }^{21}$ In line with Prediction 4 , the coefficients of $\lambda_{\mathrm{D}}$ and $\lambda_{\mathrm{A}}$ have positive coefficients in NI, but only $\lambda_{\mathrm{A}}$ is statistically significant. The coefficient of $\lambda_{A}$ switches sign (as predicted) in FI, but both $\lambda_{D}$ and $\lambda_{\mathrm{A}}$ are statistically insignificant. Hence, we only find limited support for Prediction 4.

Table 5: Determinants of efficient service provision

\begin{tabular}{|c|c|c|c|}
\hline & (1) & (2) & (3) \\
\hline EXO70 & $\begin{array}{c}-0.07 * * * \\
(0.02)\end{array}$ & $\begin{array}{c}-0.08 * * * \\
(0.02)\end{array}$ & $\begin{array}{c}-0.07 * * * \\
(0.02)\end{array}$ \\
\hline Insurance & $\begin{array}{c}-0.04 * * * \\
(0.01)\end{array}$ & $\begin{array}{c}-0.05 * * * \\
(0.01)\end{array}$ & $\begin{array}{c}-0.05 * * * \\
(0.01)\end{array}$ \\
\hline UT price vector & $\begin{array}{c}0.12 * * * \\
(0.02)\end{array}$ & $\begin{array}{c}0.12 * * * \\
(0.02)\end{array}$ & $\begin{array}{c}0.12 * * * \\
(0.02)\end{array}$ \\
\hline OT price vector & $\begin{array}{c}-0.08 * * * \\
(0.02)\end{array}$ & $\begin{array}{c}-0.08 * * * \\
(0.02)\end{array}$ & $\begin{array}{c}-0.07 * * * \\
(0.02)\end{array}$ \\
\hline Insurance $x$ EXO70 & & $\begin{array}{c}0.01 \\
(0.02)\end{array}$ & \\
\hline Period & & & $\begin{array}{c}-0.00 * * \\
(0.00)\end{array}$ \\
\hline Risk measure & & & $\begin{array}{l}-0.01 \\
(0.01)\end{array}$ \\
\hline$\lambda_{D}$ & & & $\begin{array}{c}0.04 \\
(0.03)\end{array}$ \\
\hline$\lambda_{A}$ & & & $\begin{array}{c}0.02 \\
(0.03)\end{array}$ \\
\hline Female & & & $\begin{array}{c}0.02 \\
(0.03)\end{array}$ \\
\hline Age & & & $\begin{array}{c}0.00 \\
(0.00)\end{array}$ \\
\hline $\mathrm{N}$ & 4704 & 4704 & 3792 \\
\hline
\end{tabular}

Notes: Dependent variable is Follow, equal to 1 if the expert followed the received signal and 0 otherwise. The table presents marginal effects estimates from Probit regressions with subject random effects. Standard errors are clustered at the matching group level and stated in parentheses. Insurance is equal to 1 if the consumer was insured in a particular period. EXO70 is equal to 1 for the EXO70 treatment. UT price vector is equal to 1 if the price for the LQS is 60 and the price for the HQS is 80 . OT price vector is equal to 1 if the price for the LQS is 60 and the price for the HQS is 120 . The omitted benchmark is the EM vector with the price of 60 for LQS and of 100 for HQS. The Risk Measure and the prosociality parameters $\lambda_{\mathrm{D}}$ and $\lambda_{\mathrm{A}}$ are defined as in the notes to Table $4 .{ }^{*} \mathrm{p}<0.10,{ }^{* *} \mathrm{p}<0.05,{ }^{* * *} \mathrm{p}<0.01$.

${ }^{21}$ Available as supplementary material at https://osf.io/9kwja/?view_only=fc4ec287dd4144d79bee2d688add23ab. 
Result 1 (Provision behavior): Predictions 1-3 are all supported by the experimental data. Experts are more likely to decide for the efficient service provision (by following the signal) when diagnostic precision is higher and with the undertreatment price vector, and they are less likely to do so when insurance is in place and with the overtreatment price vector. Prediction 4 is not unanimously supported: Our measures of prosociality affect the provision behavior by experts only partially.

\subsection{Impact of Prices and Insurance on Investment in Diagnostic Precision (ENDO)}

We now turn to predictions 5 and 6 regarding investment in diagnostic precision by experts in ENDO. In this treatment the expert chooses between six different levels of diagnostic precision. The distribution of choices is shown in Table 6 (along with the costs in ECU for each choice). Overall (i.e., pooling ENDO-NI and ENDO-FI), a precision level of $80 \%$ is both the median value and the modal choice of experts. At this level, diagnostic precision costs are 3.6 ECU. By contrast, efficiency would prescribe to acquire the $100 \%$ precise signal at the cost of $10 \mathrm{ECU}$.

According to Prediction 5, experts should invest more in diagnostic precision with the UT than with the EM price vector, and they should invest less with the OT price vector. This prediction is not supported by our data: Overall, experts invest slightly more tokens with the UT than with the EM price vector (3.80 compared to 3.56), but the difference is insignificant ( $p=0.403$, Mann-Whitney $\mathrm{U}$ test). Investment in precision is even higher with the OT (4.07) than with the EM price vector $(\mathrm{p}<0.01$, Mann-Whitney $U$ test $)$.

Table 6: Investment in diagnostic precision

\begin{tabular}{ccccc}
\hline \hline $\begin{array}{c}\text { Diagnostic } \\
\text { precision (in \%) }\end{array}$ & $\begin{array}{c}\text { Cost in } \\
\text { ECU }\end{array}$ & ENDO-NI & ENDO-FI & Pooled \\
\hline 50 & 0 & 16.12 & 20.74 & 18.43 \\
60 & 0.4 & 4.44 & 5.89 & 5.16 \\
70 & 1.6 & 17.93 & 16.39 & 17.16 \\
80 & 3.6 & 28.99 & 26.27 & 27.63 \\
90 & 6.4 & 18.03 & 17.75 & 17.89 \\
100 & 10 & 14.49 & 12.95 & 13.72 \\
\hline
\end{tabular}

Notes: The table reports the relative frequencies (in \%) with which the six different levels of diagnostic precision have been chosen by experts.

Prediction 6 states that insurance coverage for consumers leads to a reduction in experts' investment in diagnostic precision. Indeed, in periods with insurance, experts choose significantly lower precision levels than when insurance is not in place $(75.3 \%$ vs. $77.2 \%, p=$ 
0.037, $\chi^{2}$ test). Comparing the distribution of chosen precision between the third and fourth column of Table 6 further reveals that a zero investment in precision is much more frequent when insurance is in place ( 20.7 vs. $16.1 \%, p<0.01, \chi^{2}$ test), while precision levels higher than $60 \%$ are chosen more frequently in ENDO-NI than in ENDO-FI $(79.4 \%$ vs. $73.4 \%, p<0.01$, $\chi^{2}$ test).

Table 7: Determinants of investment in diagnostic precision

\begin{tabular}{|c|c|c|c|c|}
\hline & (1) & (2) & (3) & (4) \\
\hline Insurance & $\begin{array}{c}-0.13 * * \\
(0.06)\end{array}$ & $\begin{array}{c}-0.15^{* * *} \\
(0.07)\end{array}$ & $\begin{array}{c}-0.29 * * \\
(0.14)\end{array}$ & $\begin{array}{l}-0.33^{*} \\
(0.17)\end{array}$ \\
\hline UT price vector & $\begin{array}{c}0.16 \\
(0.11)\end{array}$ & $\begin{array}{c}0.19 \\
(0.12)\end{array}$ & $\begin{array}{c}0.24 \\
(0.28)\end{array}$ & $\begin{array}{c}0.27 \\
(0.31)\end{array}$ \\
\hline OT price vector & $\begin{array}{c}0.18 * * * \\
(0.05)\end{array}$ & $\begin{array}{c}0.19 * * * \\
(0.06)\end{array}$ & $\begin{array}{c}0.51 * * * \\
(0.12)\end{array}$ & $\begin{array}{c}0.53 * * * \\
(0.14)\end{array}$ \\
\hline Period & & $\begin{array}{c}-0.02 * * * \\
(0.01)\end{array}$ & & $\begin{array}{c}-0.05^{* * *} \\
(0.01)\end{array}$ \\
\hline Risk measure & & $\begin{array}{l}-0.03 \\
(0.05)\end{array}$ & & $\begin{array}{l}-0.08 \\
(0.12)\end{array}$ \\
\hline$\lambda_{D}$ & & $\begin{array}{c}0.53 * * \\
(0.24)\end{array}$ & & $\begin{array}{c}-1.67 * * * \\
(0.63)\end{array}$ \\
\hline$\lambda_{D}$ & & $\begin{array}{c}0.26 \\
(0.33)\end{array}$ & & $\begin{array}{l}-1.00 \\
(0.84)\end{array}$ \\
\hline Female & & $\begin{array}{l}-0.14 \\
(0.22)\end{array}$ & & $\begin{array}{l}-0.41 \\
(0.57)\end{array}$ \\
\hline Age & & $\begin{array}{c}0.02 \\
(0.02)\end{array}$ & & $\begin{array}{c}0.05 \\
(0.05)\end{array}$ \\
\hline Constant & & & $\begin{array}{c}3.70 * * * \\
(0.24)\end{array}$ & $\begin{array}{c}3.06^{* *} \\
(1.35)\end{array}$ \\
\hline $\mathrm{N}$ & 2208 & 1584 & 2208 & 1584 \\
\hline
\end{tabular}

Notes: Dependent variable is number of invested ECU in diagnostic precision, ranging from 0 to 10 . The table presents subject random effects regressions, using ordered Probit in columns (1) and (2) and Ordinary Least Squares in columns (3) and (4). Standard errors are clustered at the matching group level and stated in parentheses. The independent variables are defined as in Table 5. ${ }^{*} \mathrm{p}<0.10,{ }^{* *} \mathrm{p}<0.05,{ }^{* * *} \mathrm{p}<0.01$.

The regressions in Table 7 provide further support for the effect of insurance on endogenously chosen diagnostic precision. The dependent variable in these regressions is the armount of ECU invested by experts into precision in ENDO, ranging from 0 to 10 . The independent variables are the same as in the regressions of the previous subsection (excluding the treatment dummy EXO70 and its interaction with insurance). The first two columns here present results from ordered Probit regressions and the last two columns from OLS regressions. Regarding Predictions 5 and 6, the key things to note are the significantly negative coefficient on Insurance and the significantly positive coefficient for the OT price vector. Moreover, as indicated by the significantly positive coefficients for the expert's $\lambda_{D}$ parameter in columns (2) 
and (4), the more prosocial an expert is in the domain of disadvantageous inequality, the more she invests in a precise signal. This provides direct support for Prediction $7 .{ }^{22}$

Result 2 (Investment in diagnostic precision): Prediction 5 is refuted by the data, since investment in diagnostic precision by experts is higher with the overtreatment than with the equal markup price vector. Predictions 6 and 7 are confirmed: Insurance leads to a reduction in experts' investments and to lower precision, and more prosocial experts invest more in diagnostic precision.

\subsection{Market Entry by Consumers}

Market entry rates by consumers are generally very high, with a mean of $92.4 \%$ across all periods and treatments. Mean entry rates are shown in Figure 10. In line with Prediction 8(a), we find that market entry is most likely with the UT price vector, intermediate with the EM price vector, and lowest with the OT price vector. ${ }^{23}$ This holds for both EXO-treatments and ENDO, and the differences between EM and OT price vectors are always significant ( $p<$ $0.01, \chi^{2}$-tests), while the comparison of UT and EM price vectors fails significance.

Figure 10: Market entry rates, by treatment

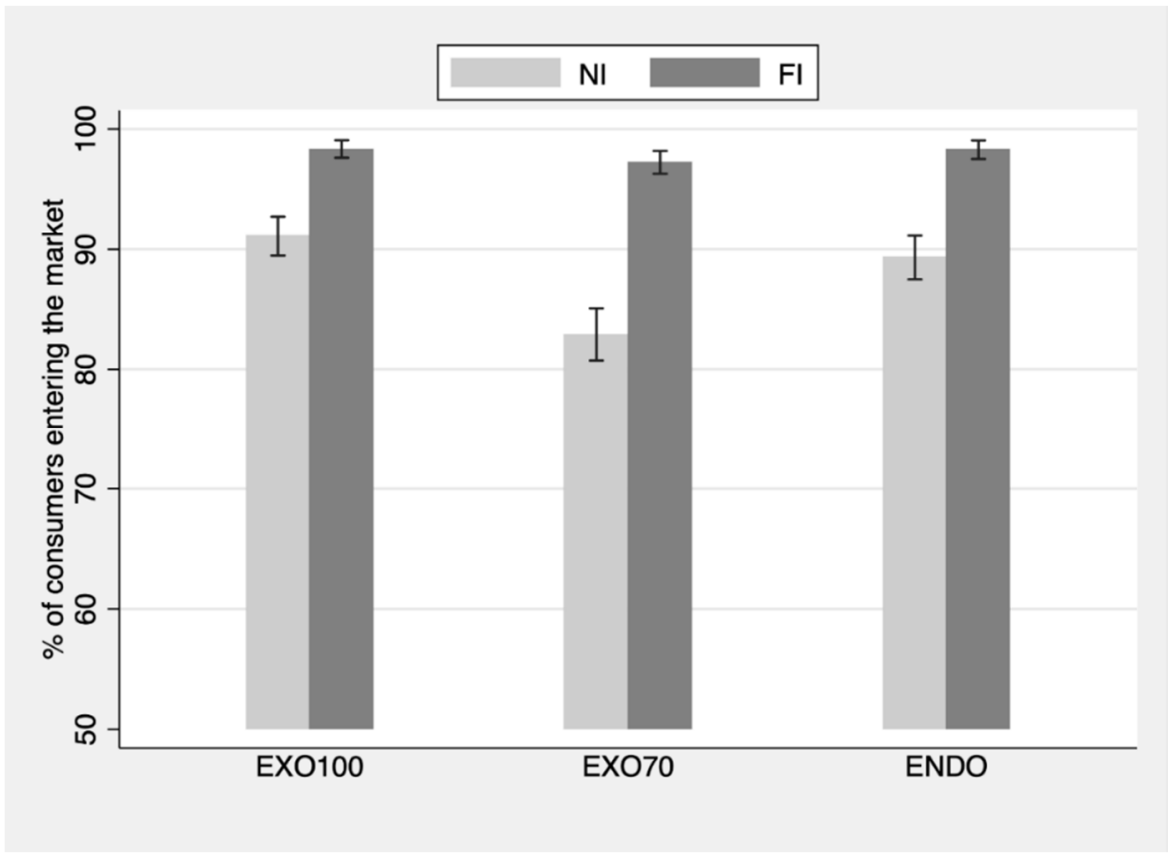

Note: All bars in the figure include $95 \%$ confidence intervals.

\footnotetext{
${ }^{22}$ The coefficient on $\lambda_{A}$ is also positive, but insignificant. The fact that Prediction 7 is more strongly borne out in the domain of disadvantageous inequality $\left(\lambda_{D}\right)$ is not unexpected, given that experts generally earn substantially less than consumers in all treatments (see Table 4).

${ }^{23}$ In the (pooled) EXO treatments, the rates are $96.8 \%, 95 \%$, and $85.5 \%$ for UT, EM, and OT price vectors respectively, and for ENDO the corresponding rates are $98 \%, 96.6 \%$, and $86.8 \%$.
} 
The effects of insurance on market entry rates confirm our Prediction 8(b). Under FI, entry rates are at $98 \%$ both in EXO and ENDO, while under NI they are $87 \%$ in EXO and $89 \%$ in EXO, with the differences always significant at $p<0.01$ ( $\chi^{2}$-tests). Finally, also Prediction $8(\mathrm{c})$ is supported by the data. A higher diagnostic precision is associated with significantly higher entry rates $\left(94.7 \%\right.$ in the aggregate in EXO100 vs. $90 \%$ in EXO70, $p<0.01, \chi^{2}$-test).

The Probit regressions in Table 8 confirm Prediction 8 as well. Like in the previous regressions, we provide two parsimonious specifications with only treatment dummies and period as independent variables, and two further specifications that include additional control variables. The dependent variable in Table 8 is a dummy variable taking the value 1 if a consumer entered the market and 0 otherwise.

Table 8: Determinants of market entry

\begin{tabular}{|c|c|c|c|c|c|}
\hline & $(1)$ & $(2)$ & (3) & (4) & $(5)$ \\
\hline & $\begin{array}{c}\text { EXO70 \& } \\
\text { EXO100 }\end{array}$ & $\begin{array}{c}\text { EXO70 \& } \\
\text { EXO100 }\end{array}$ & $\begin{array}{c}\text { EXO70 \& } \\
\text { EXO100 }\end{array}$ & ENDO & ENDO \\
\hline$E X O 70$ & $\begin{array}{c}-0.04 * * * \\
(0.01)\end{array}$ & $\begin{array}{c}-0.04 * * * \\
(0.01)\end{array}$ & $\begin{array}{c}-0.04 * * * \\
(0.01)\end{array}$ & & \\
\hline Insurance & $\begin{array}{c}0.09 * * * \\
(0.01)\end{array}$ & $\begin{array}{c}0.09 * * * \\
(0.01)\end{array}$ & $\begin{array}{c}0.08 * * * \\
(0.02)\end{array}$ & $\begin{array}{c}0.07 * * * \\
(0.02)\end{array}$ & $\begin{array}{c}0.06^{* * * *} \\
(0.01)\end{array}$ \\
\hline UT price vector & $\begin{array}{l}0.02 * * \\
(0.01)\end{array}$ & $\begin{array}{l}0.02 * * \\
(0.01)\end{array}$ & $\begin{array}{c}0.02 * * \\
(0.01)\end{array}$ & $\begin{array}{c}0.01 \\
(0.01)\end{array}$ & $\begin{array}{c}0.00 \\
(0.01)\end{array}$ \\
\hline OT price vector & $\begin{array}{c}-0.08 * * * \\
(0.01)\end{array}$ & $\begin{array}{c}-0.07 * * * \\
(0.01)\end{array}$ & $\begin{array}{c}-0.08 * * * \\
(0.01)\end{array}$ & $\begin{array}{c}-0.08^{* * * *} \\
(0.01)\end{array}$ & $\begin{array}{c}-0.08 * * * \\
(0.02)\end{array}$ \\
\hline Insurance $x$ EXO70 & & & $\begin{array}{c}0.01 \\
(0.02)\end{array}$ & & \\
\hline Period & & $\begin{array}{c}0.00^{* * * *} \\
(0.00)\end{array}$ & & & $\begin{array}{c}0.00 * * * \\
(0.00)\end{array}$ \\
\hline Risk measure & & $\begin{array}{l}0.00^{*} \\
(0.00)\end{array}$ & & & $\begin{array}{c}0.01 \\
(0.00)\end{array}$ \\
\hline$\lambda_{D}$ & & $\begin{array}{c}0.01 \\
(0.01)\end{array}$ & & & $\begin{array}{l}-0.01 \\
(0.01)\end{array}$ \\
\hline$\lambda_{A}$ & & $\begin{array}{c}0.02 \\
(0.01)\end{array}$ & & & $\begin{array}{c}0.05 * * * \\
(0.02)\end{array}$ \\
\hline Female & & $\begin{array}{c}-0.02 * * \\
(0.01)\end{array}$ & & & $\begin{array}{c}0.01 \\
(0.01)\end{array}$ \\
\hline Age & & $\begin{array}{c}0.00 \\
(0.00) \\
\end{array}$ & & & $\begin{array}{l}0.00^{*} \\
(0.00)\end{array}$ \\
\hline $\mathrm{N}$ & 4704 & 3936 & 4704 & 2208 & 1608 \\
\hline
\end{tabular}

Notes: Dependent variable is Market Entry, equal to 1 if the consumer entered the market and 0 otherwise. The table presents marginal effects estimates from Probit regressions with subject random effects. Standard errors are clustered at the matching group level and stated in parentheses. The independent variables are defined as in the notes to Table 4 and Table 5. $* \mathrm{p}<0.10,{ }^{* *} \mathrm{p}<0.05,{ }^{* *} \mathrm{p}<0.01$. 
The regressions in Table 8 provide support for the comparison between the OT and the EM price vector in the second part of Prediction 8(a), with consumers being less likely to enter when prices reflect overtreatment incentives. In addition, there is support for the first part of Prediction 8(a) as well, given the significant coefficients for the UT price vector. Consumers are significantly more likely (by almost 10 percentage points) to enter the market when they are insured, in the exogenous treatments as well as in ENDO, thus confirming Prediction 8(b). With respect to the effects of diagnostic uncertainty, the significant coefficient for EXO70 indicates that consumers are about 4 percentage points less likely to enter the market when the expert receives an imprecise exogenous signal about his problem, thus confirming Prediction 8(c).

Result 3 (Market entry): Market entry rates by consumers are higher when insurance is in place, regardless of whether diagnostic precision is exogenous or not. In the exogenous case, a higher precision also increases entry rates. Market entry is less likely with the overtreatment than with the equal markup price vector. Overall, the data support Predictions $8(b)$ and $8(c)$ fully, and partly support Prediction $8(a)$.

\subsection{Market Efficiency}

As a final part of our results section, we examine what the behavior of experts and consumers implies for market efficiency. The efficiency index used in this analysis measures (in \%) which fraction of the highest possible efficiency gain is achieved in an expert-consumer interaction. The highest possible efficiency gain is the difference between the first-best joint payoff and the minimal joint payoff. In the EXO treatments the first-best joint payoff is achieved if the consumer enters the market and the expert implements Strategy C, while in the ENDO treatments it is achieved if the consumer enters the market and the expert acquires the fully informative signal and follows the signal. In all treatments the minimal joint payoff is achieved if the consumer refuses interaction on the market. ${ }^{24}$ The efficiency index is then calculated as follows: Efficiency Index = (actual joint payoff - minimal joint payoff) / (first-best joint payoff - minimal joint payoff).

Figure 11 shows efficiency levels across treatments. The figure reveals that efficiency is always (i.e., irrespective of treatment) higher under full insurance (dark bars) than under no insurance (light bars). The difference is statistically significant for all three treatments pooled $(p<0.01$, Wilcoxon signed-ranks test) and in the two exogenous treatments separately $(p<$

\footnotetext{
${ }^{24}$ The first-best joint payoff equals $v-h \bar{c}-(1-h) \underline{c}-D(1)=150-0.4 * 60-0.6 * 20-10=104$ in treatments EXO100 and ENDO, and $[1-h(1-\sigma)] \bar{v}-(1-h-\sigma+2 h \sigma) \bar{c}-(h+\sigma-2 h \sigma) \underline{c}-D(\sigma)=$ $0.88 * 150-0.46 * 60-0.54 * 20-1.6=92$ in EXO70. The minimal joint payoff is 30 (the sum of the two outside payoffs in the case of no market entry) in all treatments.
} 
0.01 in $\mathrm{EXO} 70, p<0.05$ in EXO100, Wilcoxon signed-ranks tests), while it is insignificant in $\operatorname{ENDO}(p=0.12) .^{25}$

Figure 11: Efficiency rates by treatment and insurance

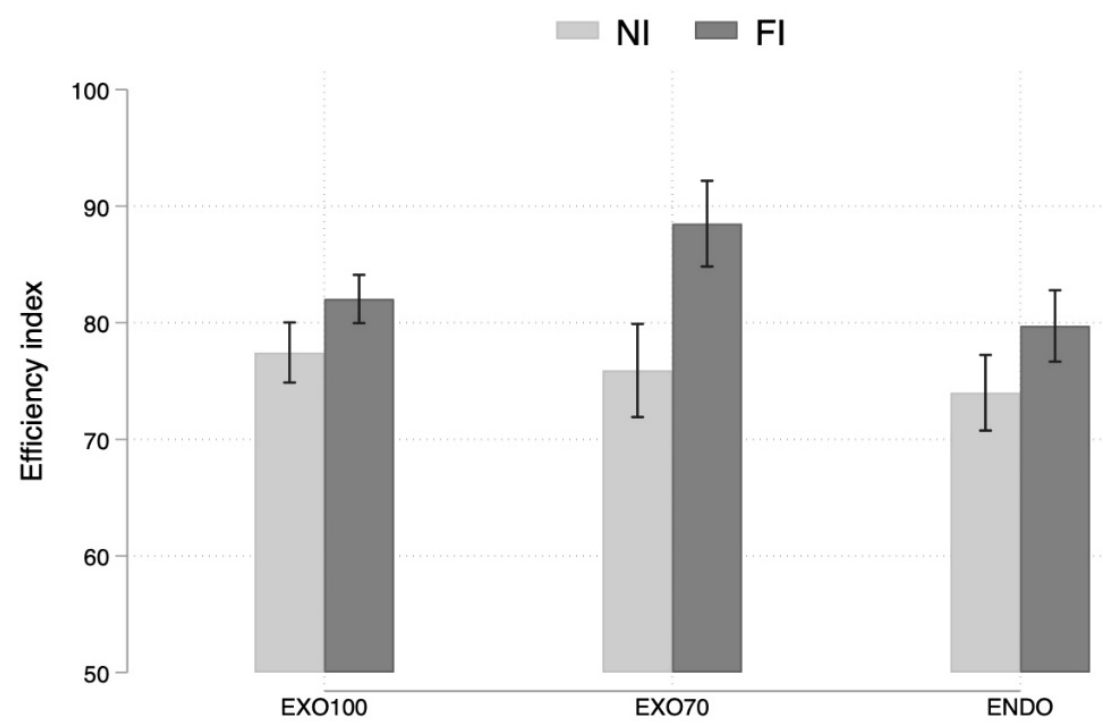

Note: All bars in the figure include $95 \%$ confidence intervals.

Table 9 presents estimates from linear regressions with the efficiency index as dependent variable. In these regressions, each consumer-expert interaction is one data point, since efficiency is measured at the market interaction level. We separate between cases with exogenous (columns 1 and 2) and endogenous (column 3) diagnostic precision. This distinction allows us to cleanly identify the effects of diagnostic precision on efficiency, but also to include the chosen level of investment in column (3).

In line with the non-parametric test results, insurance has a sizeable and significant positive impact on the efficiency index (ranging from 5 to 9 percentage points) in the exogenous as well as in the endogenous treatments. This effect is stronger in the presence of diagnostic uncertainty, as evinced by the positive and significant interaction term in (2). This analysis suggests that the drop in efficient service provision as a result of insurance documented in section 5.1 is outweighed by the increase in market entry rates documented in section 5.3, leading to a beneficial net impact of insurance coverage on efficiency.

\footnotetext{
${ }^{25}$ Comparing efficiency across treatments is less meaningful, given the way our efficiency index is constructed and the fact that its definition varies by treatment (see previous footnote). For completeness, we note that the efficiency index does not vary significantly between the two exogenous treatments, and the only significant difference is between EXO70 and ENDO ( $p<0.05$, Mann-Whitney test).
} 
Table 9: Regressions on market efficiency

\begin{tabular}{lccc}
\hline \hline & $(1)$ & $(2)$ & $(3)$ \\
Treatment(s) & EXO100 \& EXO70 & EXO100 \& EXO70 & ENDO \\
\hline Insurance & $0.09^{* * *}$ & $0.05^{* * *}$ & $0.06^{* *}$ \\
EXO70 & $(0.02)$ & $(0.02)$ & $(0.03)$ \\
& 0.02 & -0.01 & \\
UT price vector & $(0.02)$ & $(0.03)$ & \\
& 0.02 & 0.02 & -0.02 \\
OT price vector & $(0.02)$ & $(0.02)$ & $(0.03)$ \\
& $-0.12^{* * *}$ & $-0.12^{* * *}$ & $-0.08^{* * *}$ \\
Insurance x EXO70 & $(0.02)$ & $(0.02)$ & $(0.02)$ \\
& & $0.08^{* *}$ & \\
Investment & & $(0.04)$ & \\
& & & $0.02^{* * *}$ \\
Period & & & $(0.00)$ \\
& & $0.00^{* * *}$ & $0.00^{* *}$ \\
constant & $(0.00)$ & $(0.00)$ & $(0.00)$ \\
& $0.75^{* * * *}$ & $0.76^{* * *}$ & $0.65^{* * *}$ \\
$\mathrm{~N}$ & $(0.03)$ & $(0.03)$ & $(0.03)$ \\
\hline \hline
\end{tabular}

Notes: Dependent variable: Efficiency Index. The table presents random effects OLS regressions. Standard errors are clustered at the matching group level and stated in parentheses. The independent variables are defined as in the notes to Table 4 and Table $5 .{ }^{*} \mathrm{p}<0.10,{ }^{* *} \mathrm{p}<0.05,{ }^{* * *} \mathrm{p}<0.01$.

In treatment ENDO, experts' investments into diagnostic precision have a highly significant positive effect on efficiency. The intuition here is that experts who have achieved a higher precision are more likely to follow the signal and, thus, more likely to provide the correct quality. We further document an efficiency-diminishing effect of OT price vectors, which is of considerable magnitude. This finding is aligned with the way prices affect the behavior of experts in the exogenous treatments (see Prediction 1 and Result 1), with OT price vectors yielding lower rates of efficient service provision than EM price vectors.

\section{Concluding remarks}

Our paper has offered a unified theoretical framework that accommodates three important and common features of credence goods markets: (i) diagnostic uncertainty of experts when trying to identify a consumer's problem, as is frequently the case in health care or markets for repair services; (ii) insurance coverage of consumers, which is frequently the case in credence goods markets, sometimes even compulsory (as in many countries for health care services); and (iii) consumer-regarding preferences of experts, reflecting the fact that despite their informational advantage many experts in credence goods markets do not only care about their own profits, but do also consider consumer's welfare. The combination of these factors and their analysis in a unified theoretical framework is a novel contribution to the literature on credence goods 
markets. Moreover, we provide an experimental test of the model in a large laboratory experiment with 576 participants.

Our theoretical predictions and experimental findings largely coincide and reveal important effects of diagnostic uncertainty and insurance and their interaction - effects that the previous literature could not uncover. Diagnostic uncertainty reduces the rate of efficient service provision of experts in the sense that they are less likely to follow their signal about the consumer's problem. Insurance has the same effect of reducing efficient service provision. There is an important interaction effect as well. Insurance of consumers leads to a reduction in experts' investment in diagnosis and therefore to lower precision. Market entry by consumers increases with diagnostic precision and insurance and, overall, a higher precision as well as insurance coverage increase market efficiency.

We hope that our findings - although we would not want to draw too strong conclusions from a laboratory experiment - can provide some evidence-based input into the policy debate about the organization and potential regulation of credence goods markets. For instance, our finding that higher diagnostic precision increases market efficiency through reducing incentives for over- or undertreatment suggests that technological progress to improve diagnostic precision is socially welcome from an efficiency point of view. Seen from this angle, it is good news when advances in medical science make diagnoses less error-prone (Gottschalk et al., 2020) or when websites help consumers to identify their needs (Kerschbamer et al., 2019). Diagnostic precision is also linked to insurance coverage, but this relationship has never been identified before. Importantly, insurance coverage provides negative incentives for diagnostic precision, implying that regulatory policies that introduce a requirement to buy insurance may have unintended side effects on experts' effort provision to diagnose consumers' problems properly. For this reason, an important avenue for future research may be to look more deeply into what happens when consumers can only get partial insurance - meaning that they have to pay deductibles even when being insured - or when they can endogenously choose the extent of insurance. It would be interesting to study whether markets may split up in consumers who ask for insurance while others remain (voluntarily) uninsured, and how experts would respond with their diagnosis effort and tailor their offers to both types of consumers. We leave such questions for future research. 


\section{References}

Bester, H. and Dahm, M. (2018) 'Credence goods, costly diagnosis and subjective evaluation', The Economic Journal, 128(611), 1367-1394.

Bock, O., Baetge, I. and Nicklisch, A. (2014) 'Hroot: Hamburg registration and organization online Tool', European Economic Review, 71, 117-120.

Brosig-Koch, J., Hennig-Schmidt, H., Kairies-Schwarz, N. and Wiesen, D. (2016) 'Using artefactual field and lab experiments to investigate how fee-for-service and capitation affect medical service provision', Journal of Economic Behavior and Organization, 131, 17-23.

Brosig-Koch, J., Hennig-Schmidt, H., Kairies-Schwarz, N. and Wiesen, D. (2017) 'The effects of introducing mixed payment systems for physicians: Experimental evidence', Health Economics, 26(2), 243-262.

Chen, D. L., Schonger, M. and Wickens, C. (2016) 'oTree-An open-source platform for laboratory, online, and field experiments', Journal of Behavioral and Experimental Finance, 9, 88-97.

Chen, Y., Li, J. and Zhang, J. (2018), Efficient liability in expert markets. MPRA Paper No. 87317.

Dulleck, U. and Kerschbamer, R. (2006) 'On doctors, mechanics, and computer specialists: The economics of credence goods', Journal of Economic Literature, 44(1), 5-42.

Dulleck, U. and Kerschbamer, R. (2009) 'Experts vs. discounters: Consumer free-riding and experts withholding advice in markets for credence goods', International Journal of Industrial Organization, 27(1), 15-23.

Dulleck, U., Kerschbamer, R. and Sutter, M. (2011) 'The economics of credence goods: An experiment on the role of liability, verifiability, reputation, and competition', American Economic Review, 101(2), 526-555.

Fong, Y. F., Liu, T. and Wright, D. J. (2014) 'On the role of verifiability and commitment in credence goods markets', International Journal of Industrial Organization, 37, 118-129.

Gottschalk, F., Mimra, W. and Waibel, C. (2020) 'Health services as credence goods: A field experiment', The Economic Journal, 130(629), 1346-1383.

Huck, S., Lünser, G., Spitzer, F. and Tyran, J. R. (2016) 'Medical insurance and free choice of physician shape patient overtreatment: A laboratory experiment', Journal of Economic Behavior and Organization, 131, 78-105.

Hyndman, K. and Ozerturk, S. (2011) 'Consumer information in a market for expert services', Journal of Economic Behavior and Organization, 80(3), 628-640.

Inderst, R. and Ottaviani, M. (2009) 'Misselling through agents', American Economic Review, 
99(3), 883-908.

Inderst, R. and Ottaviani, M. (2012) 'Competition through commissions and kickbacks', American Economic Review, 102(2), 780-809.

Kerschbamer, R. (2015) 'The geometry of distributional preferences and a non-parametric identification approach: The Equality Equivalence Test', European Economic Review, 76, $85-103$.

Kerschbamer, R., Neururer, D. and Sutter, M. (2016) 'Insurance coverage of customers induces dishonesty of sellers in markets for credence goods', Proceedings of the National Academy of Sciences, 113(27), 7454-7458.

Kerschbamer, R., Neururer, D. and Sutter, M. (2019) 'Credence goods markets and the informational value of new media: A natural field experiment', MPI Collective Goods Discussion Paper, (2019/3).

Kerschbamer, R., Sutter, M. and Dulleck, U. (2017) 'How social preferences shape incentives in (experimental) markets for credence goods', Economic Journal, 127(600), 393-416.

Liu, F., Rasch, A., Schwarz, M. and Waibel, C. (2019) 'The role of diagnostic ability in markets for expert services', Available at SSRN 3491607.

Liu, T. (2011) 'Credence goods markets with conscientious and selfish experts', International Economic Review, 52(1), 227-244.

Liu, T. and Ma, C. to A. (2013) 'Health insurance, treatment plan, and delegation to altruistic physician', Journal of Economic Behavior and Organization, 85, 79-96.

Lu, F. (2014) 'Insurance coverage and agency problems in doctor prescriptions: Evidence from a field experiment in China', Journal of Development Economics, 106, 156-167.

Lyu, H. et al. (2017) 'Overtreatment in the United States', PLoS One 12.9: e0181970.

Mimra, W., Rasch, A. and Waibel, C. (2016) 'Price competition and reputation in credence goods markets: Experimental evidence', Games and Economic Behavior, 100, 337-352.

Pesendorfer, W. and Wolinsky, A. (2003) 'Second opinions and price competition: Inefficiency in the market for expert advice', Review of Economic Studies, 70(2), 417-437.

Schneider, H. S. (2012) 'Agency problems and reputation in expert services: evidence from auto repair', Journal of Industrial Economics, 60(3), 406-433.

Sülzle, K. and Wambach, A. (2005) 'Insurance in a market for credence goods', Journal of Risk and Insurance, 72(1), 159-176.

Wolinsky, A. (1993) 'Competition in a market for informed experts' services', RAND Journal of Economics, 24(3), 380-398. 


\section{Diagnostic Uncertainty and Insurance Coverage in Credence Goods Markets}

Loukas Balafoutas, Helena Fornwagner, Rudolf Kerschbamer, Matthias Sutter, and Maryna Tverdostup

\section{Online Appendix}




\section{Appendix A. Proof of Proposition 1: first-best provision strategy with exogenous precision}

We start by defining the generalized costs associated with each of the three provision strategies:

- $\quad$ Strategy A: $C_{A}=\bar{c}$

- $\quad$ Strategy B: $C_{B}=\underline{c}+h v$

- $\quad$ Strategy C: $\quad C_{C}=\bar{c}(1-h-\sigma+2 h \sigma)+\underline{c}(h+\sigma-2 h \sigma)+h(1-\sigma) v$.

The efficient provision strategy is the one that minimizes generalize costs. Equating $C_{A}$ and $C_{C}$ results in the hyperbola $\sigma_{A C}^{E X O-F B}$, as defined in equation (1) in the body of the paper. For $\sigma<\sigma_{A C}^{E X O-F B}$ Strategy A is more efficient than Strategy $C$ and vice versa for $\sigma>\sigma_{A C}^{E X O-F B}$. Equations $C_{B}$ and $C_{C}$ yield the hyperbola $\sigma_{B C}^{E X O-F B}$ defined in equation (2) in the paper. For $\sigma<\sigma_{B C}^{E X O-F B}$ Strategy B is more efficient than Strategy $\mathrm{C}$ and vice versa for $\sigma>\sigma_{B C}^{E X O-F B}$. Equating $C_{A}$ and $C_{B}$ yields the line $h=\frac{\tilde{c}}{v}$. For $h<\frac{\tilde{c}}{v}$ Strategy B is more efficient than Strategy A and vice versa for $h>\frac{\tilde{c}}{v}$.

Figure 1 depicts hyperbolas $\sigma_{A C}^{E X O-F B}$ and $\sigma_{B C}^{E X O-F B}$ in the $(h, \sigma)$ space, for $h \in[0,1]$ and $\sigma \in[0.5,1]$. The intercept of the two hyperbolas is found by equating $\sigma_{A C}^{E X O-F B}$ and $\sigma_{B C}^{E X O-F B}$ and the crossing point corresponds to $h=\frac{\tilde{c}}{v}$ and $\sigma=0.5$. From our assumption that $\tilde{c}<h v$ (and given that $h \in[0,1]$ ) it directly follows that $\tilde{c}<v$. Thus, $\frac{\tilde{c}}{v} \in(0,1)$ under any constellation of the parameters $\{\tilde{c}, v\}$.

As a result, the efficient provision strategy in the EXO case has the following properties:

(a) for $\sigma<\sigma_{A C}^{E X O-F B}=\frac{h(v-\tilde{c})}{(1-2 h) \tilde{c}+h v}$ and $h \in\left[\frac{\tilde{c}}{v}, 1\right]$ efficiency requires to choose Strategy A;

(b) for $\sigma<\sigma_{B C}^{E X O-F B}=\frac{(1-h) \tilde{c}}{(1-2 h) \tilde{c}+h v}$ and $h \in\left[0, \frac{\tilde{c}}{v}\right)$ efficiency requires to choose Strategy B; and

(c) for $\left\{\sigma>\sigma_{B C}^{E X O-F B}=\frac{(1-h) \tilde{c}}{(1-2 h) \tilde{c}+h v} \wedge h \in\left[0, \frac{\tilde{c}}{v}\right)\right\}$ and $\left\{\sigma>\sigma_{A C}^{E X O-F B}=\frac{h(v-\tilde{c})}{(1-2 h) \tilde{c}+h v} \wedge h \in\left[\frac{\tilde{c}}{v}, 1\right]\right\}$ efficiency requires to choose Strategy $\mathrm{C}$.

Figure 1. Optimal provision strategy with exogenous diagnostic precision.

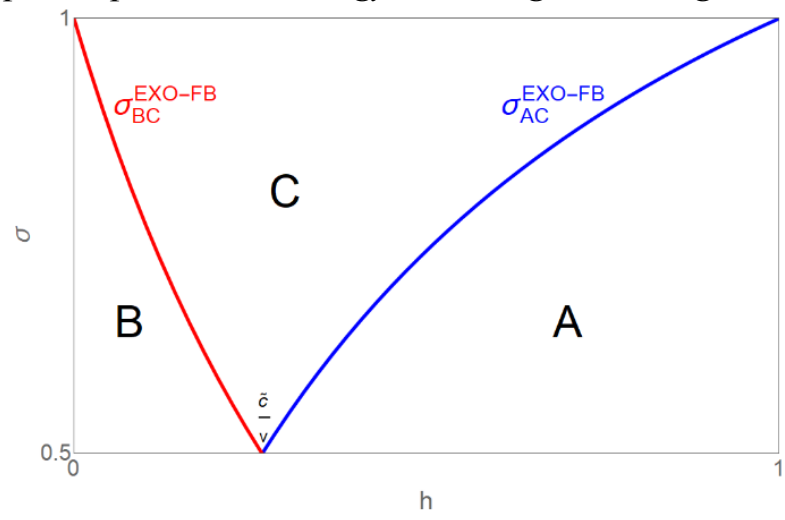

Note: In Area A, efficiency requires to implement Strategy A (blindly providing HQS without considering the diagnosis outcome); in Area B, efficiency requires to implement Strategy B (blindly providing LQS without considering the diagnosis outcome); and in Area $\mathrm{C}$, efficiency requires to implement Strategy C (following the signal). 


\section{Appendix B. Proof of Proposition 2: first-best investment in information acquisition and first- best provision strategy with endogenous precision}

The assumption that diagnostic precision can be acquired at the cost $d(\sigma-0.5)^{2}$ changes the generalized cost of Strategy $\mathrm{C}$ to $C_{C}=\bar{c}(1-h-\sigma+2 h \sigma)+\underline{c}(h+\sigma-2 h \sigma)+h(1-\sigma) v+$ $d(\sigma-0.5)^{2}$, while Strategies A and B have the same generalized costs as outlined in Appendix A.

Suppose that information acquisition (and following the signal) is efficient. Which diagnosis effort is optimal then? To address this question, we minimize $C_{C}$ with respect to $\sigma$, which yields the costminimizing diagnosis precision $\sigma^{E N D O-F B}=\min \left\{\frac{1}{2}+\frac{(1-h) \tilde{c}+h(v-\tilde{c})}{2 d}, 1\right\}$, as outlined in equation (3) in the paper. The cost-efficient diagnostic precision implies that if the diagnosis cost is relatively low, namely, $d \leq(1-h) \tilde{c}+h(v-\tilde{c})$, efficiency requires to invest in full precision, so $\sigma^{E N D O-F B}=1$. By contrast, if $d>(1-h) \tilde{c}+h(v-\tilde{c})$, efficiency entails acquiring the imperfect diagnostic precision $\sigma^{E N D O-F B}=\frac{1}{2}+\frac{(1-h) \tilde{c}+h(v-\tilde{c})}{2 d}<1$. Plugging those values into the generalized cost function for Strategy C (as outlined above) yields the generalized cost for the strategy "invest efficiently in diagnosis and follow the signal". Now, investing in diagnosis (and following the signal) is efficient if and only if the generalized cost for the strategy "invest efficiently in diagnosis and follow the signal" is lower than the generalized cost associated with Strategy A and lower than the generalized cost associated with Strategy B.

To find out under which conditions investing efficiently in diagnosis and following the signal is efficient, we define the new boundaries between strategies A, B and C, applying the same procedure as in Appendix $\mathrm{A}$. The boundary between strategies $\mathrm{A}$ and $\mathrm{C}$ is found by equating $C_{A}$ and $C_{C}$, resulting in the hyperbola $\sigma_{A C}^{E N D O-F B}$, as defined in equation (4) of the paper. The boundary between strategies $\mathrm{B}$ and $\mathrm{C}$ is found by equating $C_{B}$ and $C_{C}$, yielding hyperbola $\sigma_{B C}^{E N D O-F B}$, as defined in equation (5) in the paper. Notably, $\sigma_{A C}^{E N D O-F B}=\sigma_{B C}^{E N D O-F B}=\sigma_{A C}^{E X O-F B}=\sigma_{B C}^{E X O-F B}$ at the point $\left(h=\frac{\tilde{c}}{v}\right.$ and $\left.\sigma=0.5\right)$ for any constellation of the parameters $\{\tilde{c}, v, d\}$.

Now it is rather straightforward to show that the optimal diagnostic precision line crosses $\sigma_{\mathrm{AC}}^{\mathrm{END}-\mathrm{FB}}$ and $\sigma_{\mathrm{BC}}^{\mathrm{ENDOFB}}$ at the respective vertex, that is, $\sigma^{E N D O-F B}=\sigma_{A C}^{E N D O-F B}$ at $V \sigma_{A C}^{E N D O-F B}$ (defined by equation (6) in the paper) and $\sigma^{E N D O-F B}=\sigma_{B C}^{E N D O-F B}$ at $V \sigma_{B C}^{E N D O-F B}$ (defined by equation (7) in the paper). ${ }^{1}$

We now consider three cases, depending on the dynamics of the hyperbolas $\sigma_{A C}^{E N D O-F B}$ and $\sigma_{B C}^{E N D O-F B}$, which are summarized in panels (a), (b), and (c) of Figure 2. Let us consider them step-by-step.

Panel (a) embeds the case where the vertices of both hyperbolas $\sigma_{A C}^{E N D O-F B}$ and $\sigma_{B C}^{E N D O-F B}$ are located at $\sigma>1$, implying that they lay outside the $\sigma \in[0.5,1]$ area. Since the efficient diagnosis precision line $\sigma^{E N D O-F B}$ crosses the hyperbolas precisely at their vertices, it is straightforward that $\sigma^{E N D O-F B}=1$ if Strategy $\mathrm{C}$ is optimal. From the efficient diagnosis precision function we know that $\sigma^{E N D O-F B}=1$ iff $d \leq(1-h) \tilde{c}+h(v-\tilde{c})$. Next, we define the range of $h$ under which Strategy $\mathrm{C}$ is efficient. For that, we first search for the $h$ at which hyperbolas $\sigma_{A C}^{E N D O-F B}$ and $\sigma_{B C}^{E N D O-F B}$ intersect with the $\sigma=1$ line, which corresponds to $h=\frac{d}{4(v-\tilde{c})}$ and $h=1-\frac{d}{4 \tilde{c}}$, respectively. By looking at the dynamics of the two

\footnotetext{
${ }^{1}$ The vertices of $\sigma_{A C}^{E N D O-F B}$ and $\sigma_{B C}^{E N D O-F B}$ are found by equating the term under the square root of the respective hyperbola to 0 and solving it for $h$.
} 
hyperbolas, we conclude that the vertex of $\sigma_{B C}^{E N D O-F B}$ decreases faster than the one of $\sigma_{A C}^{E N D O-F B}$ as $d$ increases, implying that $\sigma^{E N D O-F B}=1$ for parameter constellations where Strategy $\mathrm{C}$ is efficient -as long as the vertex of $\sigma_{B C}^{E N D O-F B}$ is located at $\sigma>1$. To derive this condition, we incorporate the $h$ value at which $\sigma_{B C}^{E N D O-F B}$ crosses $\sigma=1$, i.e. $h=\frac{d}{4(v-\tilde{c})}$, into the $\sigma^{E N D O-F B}=1$ condition, i.e. $d \leq$ $(1-h) \tilde{c}+h(v-\tilde{c})$. This gives us a condition on the diagnosis cost $d$, namely $d \leq\left(1-\frac{d}{4(v-\tilde{c})}\right) \tilde{c}+$ $\frac{d}{4(v-\tilde{c})}(v-\tilde{c})$, yielding $d \leq \frac{4 \tilde{c}(v-\tilde{c})}{3 v-2 \tilde{c}}$. We therefore conclude: If the diagnosis cost $d$ is low $\left(d \leq \frac{4 \tilde{c}(v-\tilde{c})}{3 v-2 \tilde{c}}\right)$, then:

1. for $h>1-\frac{d}{4 \tilde{c}}$ efficiency requires blindly providing the HQS without investing in diagnosis (Strategy A);

2. for $h<\frac{d}{4(v-\tilde{c})}$ efficiency requires blindly providing the LQS without investing in diagnosis (Strategy B); and

3. for $h \in\left[\frac{d}{4(v-\tilde{c})}, 1-\frac{d}{4 \tilde{c}}\right]$ efficiency requires acquiring precision level $\sigma^{E N D O-F B}=1$ and following the signal.

Panel (b) covers the case where the vertex of $\sigma_{B C}^{E N D O-F B}$ falls in $\sigma \in[0.5,1]$ while the vertex of $\sigma_{A C}^{E N D O-F B}$ is still at $\sigma>1$. The former condition implies $d>\frac{4 \tilde{c}(v-\tilde{c})}{3 v-2 \tilde{c}}$ while the latter implies $d \leq \frac{4 \tilde{c}(v-\tilde{c})}{v+2 \tilde{c}}$ (derived by inserting the value of $h$ at which $\sigma_{A C}^{E N D O-F B}$ crosses $\sigma=1$, i.e. $h=1-\frac{d}{4 \tilde{c}}$, into the $\sigma^{E N D O-F B}=1$ condition, i.e. $\left.d \leq\left(1-1+\frac{d}{4 \tilde{c}}\right) \tilde{c}+\left(1-\frac{d}{4 \tilde{c}}\right)(v-\tilde{c})\right)$. We therefore conclude: If the diagnosis cost $d$ is in an intermediate range $\left(d \in\left[\frac{4 \tilde{c}(v-\tilde{c})}{3 v-2 \tilde{c}}, \frac{4 \tilde{c}(v-\tilde{c})}{v+2 \tilde{c}}\right]\right)$, then:

1. for $h>1-\frac{d}{4 \tilde{C}}$ efficiency requires blindly providing the HQS without investing in diagnosis (Strategy A);

2. for $h<\frac{\tilde{c}(2 \tilde{c}-v)-d v+\sqrt{d\left(4 \tilde{c}^{2}(2 \tilde{c}-3 v)+v^{2}(4 \tilde{c}+d)\right)}}{(v-2 \tilde{c})^{2}}$ efficiency requires blindly providing the LQS without investing in diagnosis (Strategy B); and

3. for $h \in\left[\frac{\tilde{c}(2 \tilde{c}-v)-d v+\sqrt{d\left(4 \tilde{c}^{2}(2 \tilde{c}-3 v)+v^{2}(4 \tilde{c}+d)\right)}}{(v-2 \tilde{c})^{2}}, 1-\frac{d}{4 \tilde{c}}\right]$ efficiency requires acquiring precision level $\sigma^{E N D O-F B}=1$ and following the signal for $d \leq(1-h) \tilde{c}+h(v-\tilde{c})$ and acquiring precision level $\sigma^{E N D O-F B}=\frac{1}{2}+\frac{(1-h) \tilde{c}+h(v-\tilde{c})}{2 d}$ and following the signal for $d>(1-h) \tilde{c}+$ $h(v-\tilde{c})$.

Panel (c) refers to the case where the vertices of hyperbolas $\sigma_{A C}^{E N D O-F B}$ and $\sigma_{B C}^{E N D O-F B}$ fall into the $\sigma \in$ $[0.5,1]$ range. The latter happens when diagnosis cost gets higher than $\frac{4 \tilde{c}(v-\tilde{c})}{v+2 \tilde{c}}$. We, therefore, conclude: If the diagnosis cost $d$ is large $\left(d>\frac{4 \tilde{c}(v-\tilde{c})}{v+2 \tilde{c}}\right)$, then:

1. for $h>\frac{\tilde{c}(2 \tilde{c}-v)+d v-\sqrt{d\left(4 \tilde{c}^{2}(3 v-2 \tilde{c})+v^{2}(d-4 \tilde{c})\right)}}{(v-2 \tilde{c})^{2}}$ efficiency requires blindly providing the HQS without investing in diagnosis (Strategy A); 
2. for $h<\frac{\tilde{c}(2 \tilde{c}-v)-d v+\sqrt{d\left(4 \tilde{c}^{2}(2 \tilde{c}-3 v)+v^{2}(4 \tilde{c}+d)\right)}}{(v-2 \tilde{c})^{2}}$ efficiency requires blindly providing the LQS without investing in diagnosis (Strategy B); and

3. for $h \in\left[\frac{\tilde{c}(2 \tilde{c}-v)-d v+\sqrt{d\left(4 \tilde{c}^{2}(2 \tilde{c}-3 v)+v^{2}(4 \tilde{c}+d)\right)}}{(v-2 \tilde{c})^{2}}, \frac{\tilde{c}(2 \tilde{c}-v)+d v-\sqrt{d\left(4 \tilde{c}^{2}(3 v-2 \tilde{c})+v^{2}(d-4 \tilde{c})\right)}}{(v-2 \tilde{c})^{2}}\right]$ efficiency requires acquiring precision level $\sigma^{E N D O-F B}=\frac{1}{2}+\frac{(1-h) \tilde{c}+h(v-\tilde{c})}{2 d}$ and following the signal.

Figure 2. Optimal provision strategy with choice of diagnostic precision in ENDO.

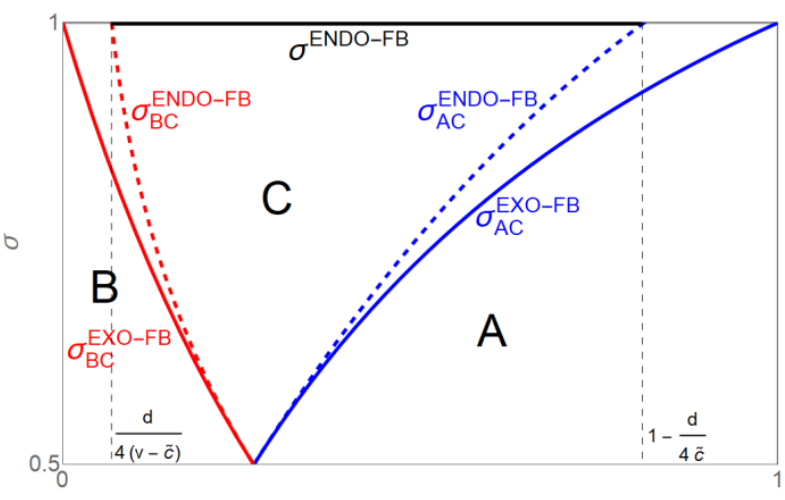

(a) $d \leq \frac{4 \tilde{c}(v-\tilde{c})}{3 v-2 \tilde{c}}$

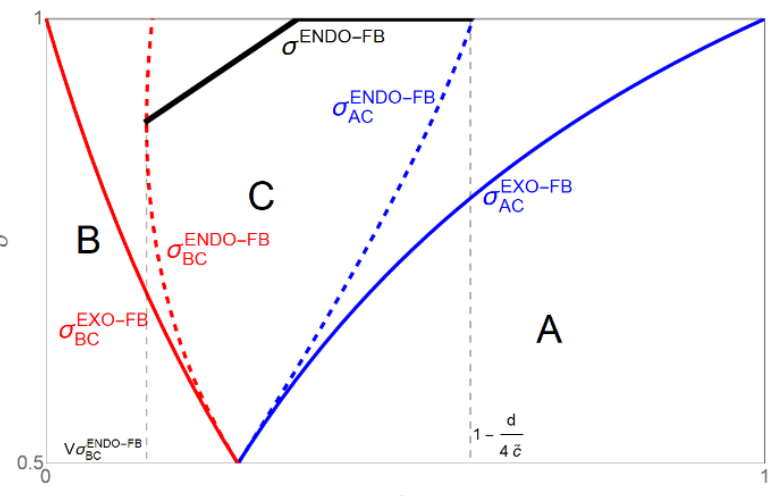

(b) $\frac{4 \tilde{c}(v-\tilde{c})}{3 v-2 \tilde{c}}<d<\frac{4 \tilde{c}(v-\tilde{c})}{v+2 \tilde{c}}$

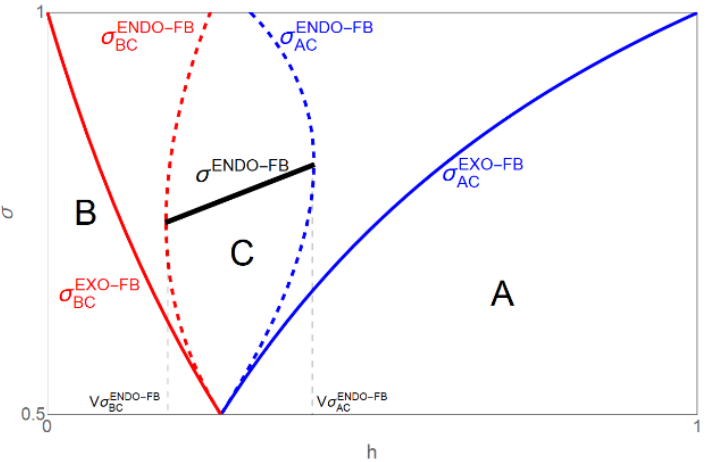

(c) $d \geq \frac{4 \tilde{c}(v-\tilde{c})}{v+2 \tilde{c}}$

Note: In each of the three panels investing in diagnostic precision and following the signal is efficient for those values of $h$ covered by the solid black line. This solid black line also gives the efficient precision level. For $h$ values to the left of the (start of the) solid black line efficiency requires to implement Strategy B; and for $h$ values to the right of the (end of the) black solid line efficiency requires to implement Strategy A. The solid red and the solid blue line are the functions $\sigma_{A C}^{E X O-F B}$ and $\sigma_{B C}^{E X O-F B}$ defined in equations (1) and (2); the dashed red and the dashed blue line are the functions $\sigma_{A C}^{E N D O-F B}$ and $\sigma_{B C}^{E N D O-F B}$ defined in equations (4) and (5); the solid black line is the function $\sigma^{E N D O-F B}$ defined in equation (3); and the dashed vertical lines are the vertices of the functions $\sigma_{A C}^{E N D O-F B}$ and $\sigma_{B C}^{E N D O-F B}$ defined in equations (6) and (7). 


\section{Appendix C. Proof of Proposition 3: provision strategy with exogenous precision and no insurance}

Similarly to propositions 1 and 2, the expert has the choice between the three pure strategies outlined in the paper. The fourth pure strategy - Strategy D, where the expert provides a treatment opposite to the signal she receives - is dominated by one of the other three strategies for any given constellation of the parameters $\{\tilde{p}, \tilde{c}, h, v, t\}$. We show this by first ignoring Strategy $\mathrm{D}$ and deriving the provision areas for the case where only strategies $\mathrm{A}, \mathrm{B}$ and $\mathrm{C}$ are available. Later we show that in the area where Strategy $X \in\{A, B, C\}$ is preferred to the other two strategies, Strategy $\mathrm{X}$ is also preferred to Strategy $\mathrm{D}$.

To characterize the expert's provision policy for the case where only strategies A, B and C are available, we compare the payoffs for the expert associated with each of the three strategies. By equating the payoffs associated with strategies $\mathrm{A}$ and $\mathrm{C}$ (as specified in the paper) we get the boundary condition $\sigma_{A C}^{E X O-N I}=f(\lambda, h, \tilde{p}, \tilde{c}, v, t)=\frac{h((1-\lambda) \tilde{p}-\tilde{c})+h \lambda(v-t)+h t}{(1-2 h)(\tilde{c}-(1-\lambda) \tilde{p})+h \lambda(v-t)+h t} \quad$ or $\quad \lambda_{A C}^{E X O-N I}=f(\sigma, h, \tilde{p}, \tilde{c}, v, t)=$ $\frac{(h+\sigma-2 h \sigma)(\tilde{p}-\tilde{c})+h(1-\sigma) t}{(h+\sigma-2 h \sigma) \tilde{p}-h(1-\sigma)(v-t)}$. By equating the payoffs associated with strategies B and C (as specified in the paper) we get the boundary condition $\sigma_{B C}^{E X O-N I}=f(\lambda, h, \tilde{p}, \tilde{c}, v, t)=\frac{(1-h)(\tilde{c}-(1-\lambda) \tilde{p})}{(1-2 h)(\tilde{c}-(1-\lambda) \tilde{p})+h \lambda(v-t)+h t}$ or $\lambda_{B C}^{E X O-N I}=f(\sigma, h, \tilde{p}, \tilde{c}, v, t)=\frac{(1-h-\sigma+2 h \sigma)(\tilde{p}-\tilde{c})+h \sigma t}{(1-h-\sigma+2 h \sigma) \tilde{p}-h \sigma(v-t)}$.

Functions $\sigma_{A C}^{E X O-N I}$ and $\sigma_{B C}^{E X O-N I}$ are hyperbolas. To define the areas in the $(\lambda, \sigma)$ space in which each of the three strategies is optimal for the expert, we first derive the vertices of the two hyperbolas. The vertex of hyperbola $\sigma_{A C}^{E X O-N I}$ is $V \lambda_{A C}^{E X O-N I}=\frac{h(1-h)(v(\tilde{p}-\tilde{c})+\tilde{c} t)}{((2 h-1) \tilde{p}-h(v-t))^{2}}$, while the vertex of hyperbola $\sigma_{B C}^{E X O-N I}$ is $V \lambda_{B C}^{E X O-N I}=\frac{-h(1-h)(v(\tilde{p}-\tilde{c})+\tilde{c} t)}{((2 h-1) \tilde{p}-h(v-t))^{2}}$.

From these expressions, it is easy to see that the two hyperbolas are geometrically similar and mirroring each other. Furthermore, the respective hyperbola does not exist when the associated vertex is 0 . The latter is true iff $\tilde{p}=\frac{\tilde{c}(v-t)}{v}$. Consequently, when $\tilde{p}=\frac{\tilde{c}(v-t)}{v}$, the vertex $V \lambda_{A C}^{E X O-N I}$ changes from positive to negative (i.e., the curve changes from concave to convex), while the vertex $V \lambda_{B C}^{E X O-N I}$ changes vice versa (i.e., the curve changes from convex to concave).

Next, let us derive the boundary values of functions $\lambda_{A C}^{E X O-N I}, \sigma_{A C}^{E X O-N I}, \lambda_{B C}^{E X O-N I}$ and $\sigma_{B C}^{E X O-N I}$.

- $\quad \lambda_{B C}^{E X O-N I}(\sigma=1)=\lambda_{1}^{E X O-N I}=\frac{\tilde{c}-t-\tilde{p}}{v-t-\tilde{p}}$ is the $\lambda$-value of the function $\lambda_{B C}^{E X O-N I}$ when $\sigma=1$.

- $\lambda_{B C}^{E X O-N I}(\sigma=0.5)=\lambda_{A C}^{E X O-N I}(\sigma=0.5)=\lambda_{2}^{E X O-N I}=\frac{\tilde{c}-h t-\tilde{p}}{h(v-t)-\tilde{p}}$ is the $\lambda$-value of the function $\lambda_{B C}^{E X O-N I}$ or function $\lambda_{A C}^{E X O-N I}$ when $\sigma=0.5$.

- $\quad \lambda_{A C}^{E X O-N I}(\sigma=1)=\lambda_{3}^{E X O-N I}=1-\frac{\tilde{c}}{\tilde{p}}$ is the $\lambda$-value of the function $\lambda_{A C}^{E X O-N I}$ when $\sigma=1$.

- $\sigma_{A C}^{E X O-N I}(\lambda=1)=\sigma_{1}^{E X O-N I}=\frac{1}{2}+\frac{h(v-\tilde{c})-(1-h) \tilde{c}}{2[h(v-\tilde{c})+(1-h) \tilde{c}]}$ is the $\sigma$-value of the function $\sigma_{A C}^{E X O-N I}$ when $\lambda=1$.

- $\sigma_{B C}^{E X O-N I}(\lambda=0)=\sigma_{2}^{E X O-N I}=\frac{1}{2}+\frac{(1-h)(\tilde{c}-\tilde{p})-h(\tilde{p}-\tilde{c}+t)}{2[h(\tilde{p}-\tilde{c}+t)+(1-h)(\tilde{c}-\tilde{p})]}$ is the $\sigma$-value of the function $\sigma_{B C}^{E X O-N I}$ when $\lambda=0$.

- $\sigma_{A C}^{E X O-N I}(\lambda=0)=\sigma_{3}^{E X O-N I}=\frac{1}{2}+\frac{h(\tilde{p}-\tilde{c}+t)-(1-h)(\tilde{c}-\tilde{p})}{2[h(\tilde{p}-\tilde{c}+t)+(1-h)(\tilde{c}-\tilde{p})]}$ is the $\sigma$-value of the function $\sigma_{A C}^{E X O-N I}$ when $\lambda=0$. 
- $\sigma_{B C}^{E X O-N I}(\lambda=1)=\sigma_{4}^{E X O-N I}=\frac{1}{2}+\frac{(1-h) \tilde{c}-h(v-\tilde{c})}{2[h(v-\tilde{c})+(1-h) \tilde{c}]}$ is the $\sigma$-value of the function $\sigma_{B C}^{E X O-N I}$ when $\lambda=1$.

Next, let us analyze one-by-one the locations of those boundary values on the $\lambda$-axis and the $\sigma$-axis.

1. $\lambda_{1}^{E X O-N I}=\frac{\tilde{c}-t-\tilde{p}}{v-t-\tilde{p}}:$

i. $\quad \lambda_{1}^{E X O-N I}<0$ iff $\tilde{c}-t<\tilde{p}<v-t$.

ii. $\quad \lambda_{1}^{E X O-N I} \in[0,1]$ iff $\tilde{p}<\tilde{c}-t$.

iii. $\quad \lambda_{1}^{E X O-N I}>1$ iff $\tilde{p}>v-t$.

2. $\quad \lambda_{2}^{E X O-N I}=\frac{\tilde{c}-h t-\tilde{p}}{h(v-t)-\tilde{p}}$ :

i. $\quad \lambda_{2}^{E X O-N I}<0$ iff $\tilde{c}-h t<\tilde{p}<h v$.

ii. $\quad \lambda_{2}^{E X O-N I} \in[0,1]$ iff $\tilde{p}<\tilde{c}-h t$.

iii. $\quad \lambda_{2}^{E X O-N I}>1$ iff $\tilde{p}>h v$.

3. $\lambda_{3}^{E X O-N I}=1-\frac{\tilde{c}}{\tilde{p}}$ :

i. $\quad \lambda_{3}^{E X O-N I}<0$ iff $\tilde{p}<\tilde{c}$.

ii. $\quad \lambda_{3}^{E X O-N I} \in[0,1]$ if $\tilde{c} \leq \tilde{p} \leq \tilde{p}+\tilde{c}$.

iii. $\quad \lambda_{3}^{E X O-N I}>1$ iff $\tilde{p}>\tilde{p}+\tilde{c}$.

4. $\sigma_{1}^{E X O-N I}=\frac{1}{2}+\frac{h(v-\tilde{c})-(1-h) \tilde{c}}{2[h(v-\tilde{c})+(1-h) \tilde{c}]}$ : given our assumption $\tilde{c}<h v$ we immediately get $\sigma_{1}^{E X O-N I} \in$ $[0.5,1]$.

5. $\quad \sigma_{2}^{E X O-N I}=\frac{1}{2}+\frac{(1-h)(\tilde{c}-\tilde{p})-h(\tilde{p}-\tilde{c}+t)}{2[h(\tilde{p}-\tilde{c}+t)+(1-h)(\tilde{c}-\tilde{p})]}$ :

$$
\begin{aligned}
\text { i. } & \sigma_{2}^{E X O-N I}<0.5 \text { if } \tilde{c}-h t<\tilde{p} \leq \tilde{c} . \\
\text { ii. } & \sigma_{2}^{E X O-N I} \in[0.5,1] \text { iff } \tilde{c}-t<\tilde{p}<\tilde{c}-h t . \\
\text { iii. } & \sigma_{2}^{E X O-N I}>1 \text { if } \tilde{p}>\tilde{c} .
\end{aligned}
$$

6. $\quad \sigma_{3}^{E X O-N I}=\frac{1}{2}+\frac{h(\tilde{p}-\tilde{c}+t)-(1-h)(\tilde{c}-\tilde{p})}{2[h(\tilde{p}-\tilde{c}+t)+(1-h)(\tilde{c}-\tilde{p})]}$ :

i. $\quad \sigma_{3}^{E X O-N I}<0.5$ if $\tilde{c}-t<\tilde{p}<\tilde{c}-h t$.

ii. $\quad \sigma_{3}^{E X O-N I} \in[0.5,1]$ iff $\tilde{c}-h t<\tilde{p}<\tilde{c}$.

iii. $\quad \sigma_{3}^{E X O-N I}>1 \tilde{p} \geq \tilde{c}$.

7. $\sigma_{4}^{E X O-N I}=\frac{1}{2}+\frac{(1-h) \tilde{c}-h(v-\tilde{c})}{2[h(v-\tilde{c})+(1-h) \tilde{c}]}$ : given our assumption $\tilde{c}<h v$ we immediately get $\sigma_{4}^{E X O-N I}<$ 0.5 .

Let us now consider each panel of Figure 3 in detail, accounting for the underlying condition $\tilde{c}<h v$, as well as for the additional condition $\tilde{c}>t$. We derive typical locations of the curves $\lambda_{A C}^{E X O-N I}$ and $\lambda_{B C}^{E X O-N I}$ when $\tilde{p}$ falls in the specific interval, as well as upper and lower boundaries of the respective functions.

a. Panel (a) corresponds to the case where $0<\tilde{p}<\tilde{c}-t$. Following the geometrical properties of hyperbolas $\lambda_{A C}^{E X O-N I}$ and $\lambda_{B C}^{E X O-N I}$, function $\lambda_{A C}^{E X O-N I}$ is concave and function $\lambda_{B C}^{E X O-N I}$ is convex, since $\tilde{p}<\frac{\tilde{c}(v-t)}{v}$. Both functions intersect at $\lambda_{2}^{E X O-N I}$. The price difference restriction immediately yields $\lambda_{1}^{E X O-N I} \in[0,1]$; since $\tilde{p}<\tilde{c}-t<\tilde{c}-h t, \lambda_{2}^{E X O-N I} \in[0,1]$; furthermore, $\sigma_{1}^{E X O-N I} \in$ $[0.5,1]$ and $\sigma_{4}^{E X O-N I}<0.5$. The upper and lower boundaries of the $\lambda_{A C}^{E X O-N I}$ and $\lambda_{B C}^{E X O-N I}$ functions are determined by the location of the intercepts $\lambda_{1}^{E X O-N I}, \lambda_{2}^{E X O-N I}$ and $\sigma_{1}^{E X O-N I}$ at the upper and the lower limit of $\tilde{p}$, thus at $\tilde{p}=0$ and at $\tilde{p}=\tilde{c}-t: \sigma_{1}^{E X O-N I}$ is independent of $\tilde{p}$, therefore, it remains 
constant at any $\tilde{p} ; \lambda_{1}^{E X O-N I}(\tilde{p}=0)=\frac{\tilde{c}-t}{v-t}$ and $\lambda_{1}^{E X O-N I}(\tilde{p}=\tilde{c}-t)=0 ; \lambda_{2}^{E X O-N I}(\tilde{p}=0)=\frac{\tilde{c}-h t}{h(v-t)}$ and $\lambda_{2}^{E X O-N I}(\tilde{p}=\tilde{c}-t)=\frac{(1-h) t}{h(v-t)-\tilde{c}+t}$, therefore $\lambda_{2}^{E X O-N I}(\tilde{p}=0)>\lambda_{2}^{E X O-N I}(\tilde{p}=\tilde{c}-t)$ always under the assumption $t<\tilde{c}<h v$ and both $\lambda_{1}^{E X O-N I}$ and $\lambda_{2}^{E X O-N I}$ decrease as $\tilde{p}$ increases from 0 to $\tilde{c}-t$.

b. Panel (b) corresponds to the case where $\tilde{c}-t<\tilde{p}<\tilde{c}-h t$. As in panel (a), function $\lambda_{A C}^{E X O-N I}$ is concave and function $\lambda_{B C}^{E X O-N I}$ is convex. Since $\tilde{p}<\tilde{c}-h t, \lambda_{2}^{E X O-N I} \in[0,1]$; furthermore, $\sigma_{1}^{E X O-N I} \in[0.5,1]$ and $\sigma_{4}^{E X O-N I}<0.5$. Given the price difference restriction $\tilde{p}>\tilde{c}-t, \lambda_{1}^{E X O-N I}<$ 0 and $\sigma_{2}^{E X O-N I} \in[0.5,1]$. Hence, the major difference between panel (b) and panel (a) is caused by a change in the curvature of the function $\lambda_{B C}^{E X O-N I}$ as a result of the increase in $\tilde{p}$. Consequently, the intercept $\lambda_{1}^{E X O-N I}$ moves outside of the range $\lambda \in[0,1]$ and the curve intersects the $\sigma$-axis in $\sigma_{2}^{E X O-N I}$. The upper and lower limit of the two functions are as follows: $\sigma_{2}^{E X O-N I}(\tilde{p}=\tilde{c}-t)=$ $1, \sigma_{2}^{E X O-N I}(\tilde{p}=\tilde{c}-h t)=0.5 ; \lambda_{2}^{E X O-N I}(\tilde{p}=\tilde{c}-t)=\frac{(1-h) t}{h(v-t)-\tilde{c}+t}$ and $\lambda_{2}^{E X O-N I}(\tilde{p}=\tilde{c}-h t)=0$. Therefore, both $\sigma_{2}^{E X O-N I}$ and $\lambda_{2}^{E X O-N I}$ decrease as $\tilde{p}$ moves from the lower limit to the upper limit. As in the previous sub-case $\sigma_{1}^{E X O-N I}$ remains constant.

c. Panel (c) corresponds to the case where $\tilde{c}-h t<\tilde{p}<\tilde{c}$. In this range of $\tilde{p}$, the shape of functions $\lambda_{A C}^{E X O-N I}$ and $\lambda_{B C}^{E X O-N I}$ changes at $\tilde{p}=\frac{\tilde{c}(v-t)}{v}$. As long as $\tilde{p}<\frac{\tilde{c}(v-t)}{v}, \lambda_{A C}^{E X O-N I}$ is concave and $\lambda_{B C}^{E X O-N I}$ is convex. At $\tilde{p}=\frac{\tilde{c}(v-t)}{v}$, both functions are straight lines. At $\tilde{p}>\frac{\tilde{c}(v-t)}{v}, \lambda_{B C}^{E X O-N I}$ is concave and $\lambda_{A C}^{E X O-N I}$ is convex. However, since $\tilde{p}>\tilde{c}-h t, \lambda_{2}^{E X O-N I}<0$ and the $\lambda_{B C}^{E X O-N I}$ curve does not appear in the $\lambda \in[0,1]$ interval. Since $\tilde{c}-h t<\tilde{p}<\tilde{c}, \sigma_{3}^{E X O-N I} \in[0.5,1] ; \sigma_{1}^{E X O-N I} \in$ $[0.5,1]$ always. When $\tilde{p}$ is at its lower limit, $\sigma_{3}^{E X O-N I}(\tilde{p}=\tilde{c}-h t)=0.5$. When $\tilde{p}$ is at its upper limit, $\sigma_{3}^{E X O-N I}(\tilde{p}=\tilde{c})=1$. Therefore, $\sigma_{3}^{E X O-N I}$ increases as $\tilde{p}$ increases from the lower to the upper limit, while $\sigma_{1}^{E X O-N I}$ remains constant.

d. Panel (d) corresponds to the case where $\tilde{p}>\tilde{c}$. In this range of $\tilde{p}, \lambda_{A C}^{E X O-N I}$ remains convex and $\lambda_{B C}^{E X O-N I}$ remains concave. However, since $\lambda_{2}^{E X O-N I}<0$ for $\tilde{p}>\tilde{c}$, the $\lambda_{B C}^{E X O-N I}$ curve remains outside the $\lambda \in[0,1]$ range. Since $\tilde{p}>\tilde{c}, \lambda_{1}^{E X O-N I} \in[0,1] ; \sigma_{1}^{E X O-N I} \in[0.5,1]$ always. At the lower limit of $\tilde{p}(\tilde{p}=\tilde{c}), \lambda_{3}^{E X O-N I}(\tilde{p}=\tilde{c})=0$, and $\lambda_{3}^{E X O-N I}$ increases as $\tilde{p}$ increases. As in the previous sub-case $\sigma_{1}^{E X O-N I}$ remains constant.

We complete the proof by showing that Strategy D, where the expert provides a treatment opposite to the signal she receives, is dominated by one of the other three strategies for any given parameter constellation. Strategy D is associated with the following utility for the expert: $\Pi_{D}=(h+\sigma-2 h \sigma) \bar{\Delta}+$ $(1-h-\sigma+2 h \sigma) \underline{\Delta}-h \sigma t+\lambda[(1-h \sigma) v-\underline{p}(1-h-\sigma+2 h \sigma)-\bar{p}(h+\sigma-2 h \sigma)+h \sigma t]$. First consider panel (a) of Figure 3. In this panel Strategy A is strictly preferred over the other two strategies for constellations satisfying $\lambda>\lambda_{2}^{E X O-N I}$ and $\sigma<\sigma_{A C}^{E X O-N I}$. We now show that for all $\lambda>\lambda_{2}^{E X O-N I}$ we have $\Pi_{A}>\Pi_{D}$. To see this note that $\Pi_{A}-\Pi_{D}=(1-h-\sigma+2 h \sigma)(\tilde{p}-\tilde{c})+(1-\lambda) \tilde{p}+\lambda h \sigma(v-$ $t)+h \sigma t$, which is zero at $\left(\lambda=\lambda_{2}^{E X O-N I}\right.$ and $\left.\sigma=0.5\right)$ and strictly increasing in $\lambda$ and $\sigma$. Next note that Strategy $\mathrm{B}$ is strictly preferred over the other two strategies for constellations satisfying $\lambda<$ $\lambda_{2}^{E X O-N I}$ and $\sigma<\sigma_{B C}^{E X O-N I}$. For $\lambda<\lambda_{2}^{E X O-N I}$ we have $\Pi_{B}>\Pi_{D}$ since $\Pi_{B}-\Pi_{D}=(h+\sigma-$ $2 h \sigma)(\tilde{c}-\tilde{p})+\lambda(\sigma-2 h \sigma-h) \tilde{p}+h(1-\sigma)(\lambda(t-v)-t)$ is zero at $\left(\lambda=\lambda_{2}^{E X O-N I}\right.$ and $\left.\sigma=0.5\right)$ and strictly decreasing in $\lambda$ and strictly increasing in $\sigma$. Finally note that $\Pi_{C}-\Pi_{D}=$ $(1-\sigma)((\tilde{p}-\tilde{c})(1-2 h)-\lambda(1-2 h) \tilde{p}-\lambda h(v-t)-h t)$ is zero at $\left(\lambda=\lambda_{2}^{E X O-N I}\right.$ and $\left.\sigma=0.5\right)$ and strictly increasing in $\lambda$ and $\sigma$. Thus, in panel (a) of Figure 3 Strategy D is dominated by at least one 
of the other three strategies for any parameter constellation. The proof for panels (b), (c) and (d) is similar and available upon request.

Figure 3. Expert provision behavior with exogenous precision and no insurance (EXO-NI).
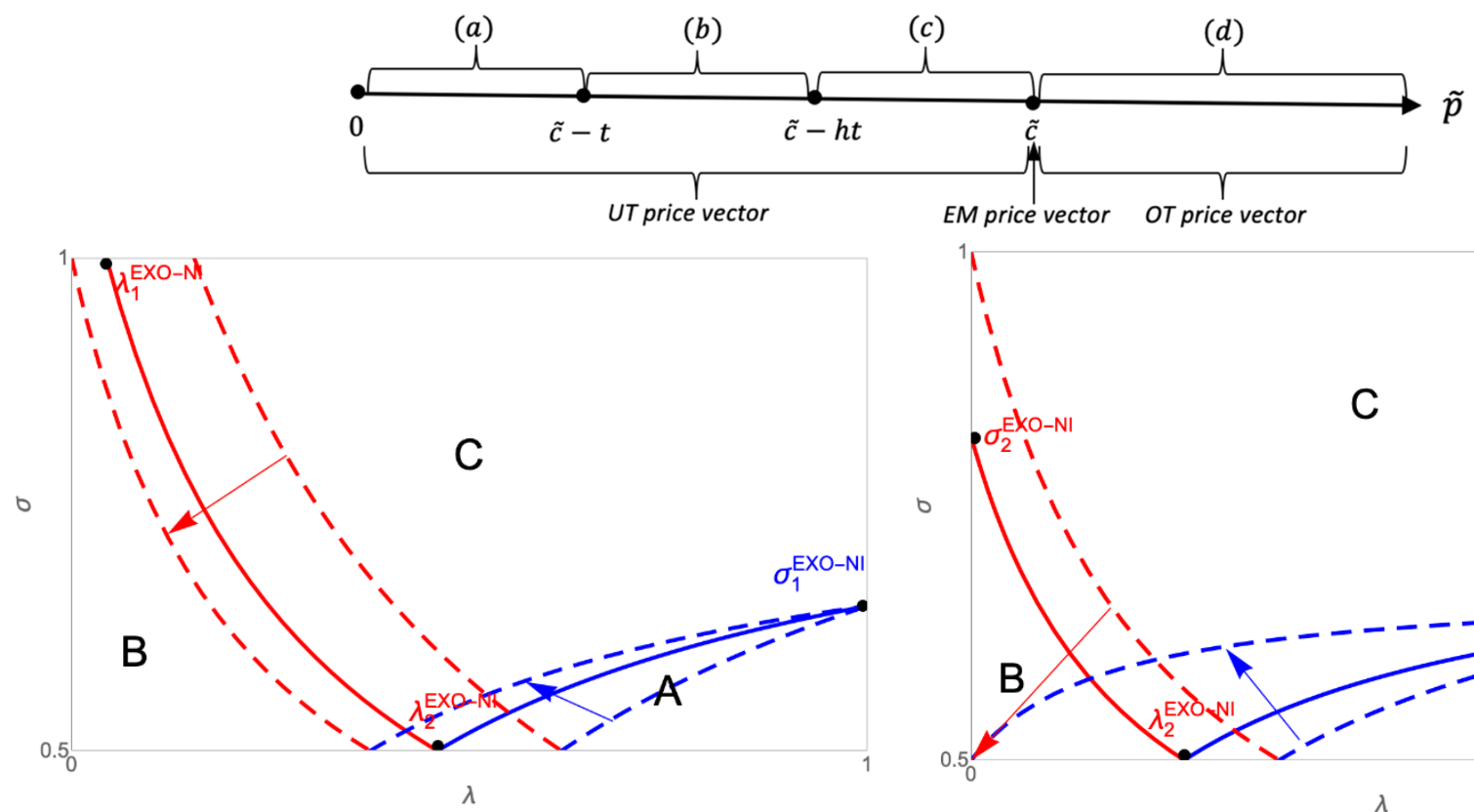

(a) $0<\tilde{p} \leq \tilde{c}-t$

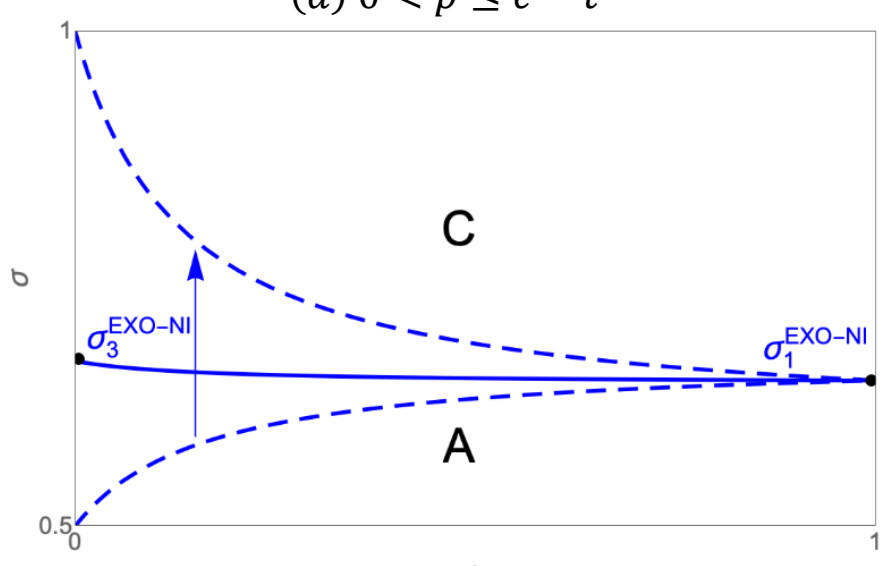

(c) $\tilde{c}-h t<\tilde{p}<\tilde{c}$

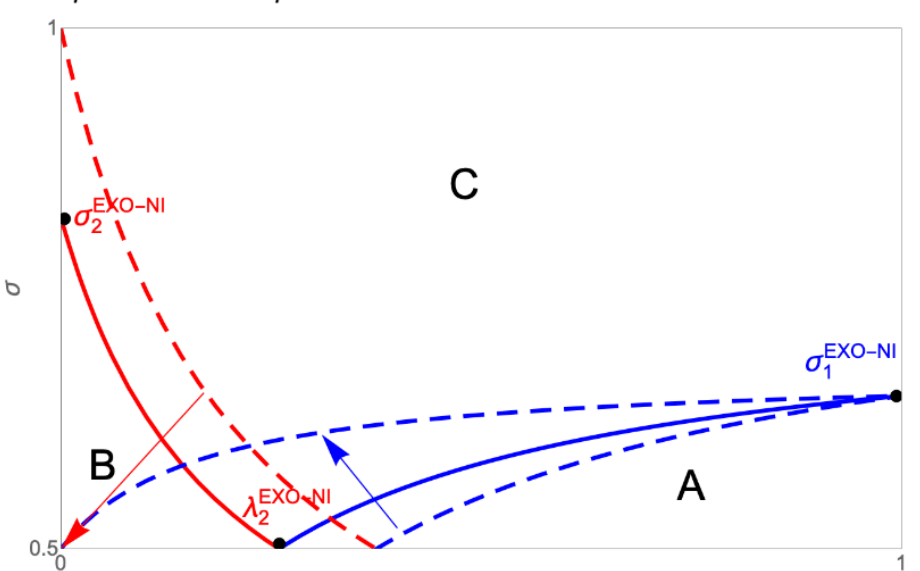

(b) $\tilde{c}-t<\tilde{p} \leq \tilde{c}-h t$

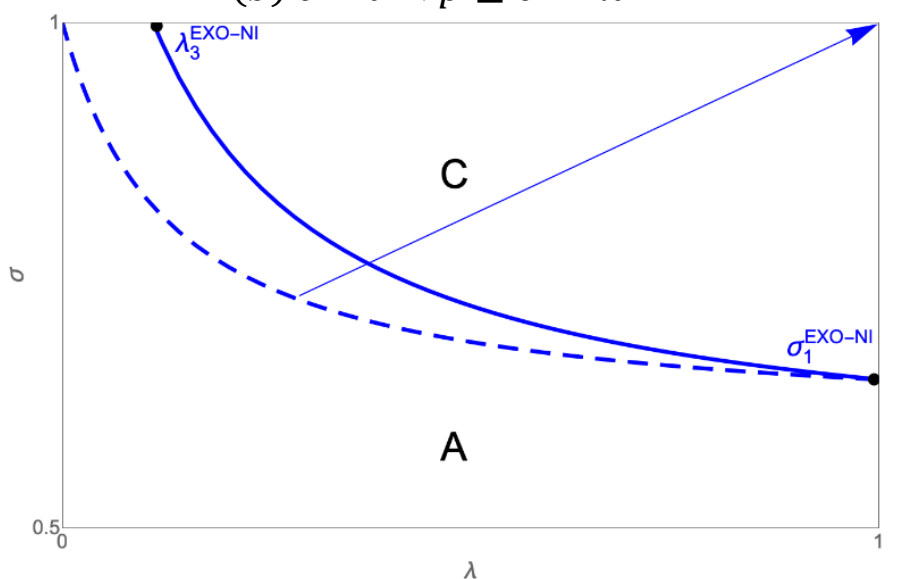

(d) $\tilde{p} \geq \tilde{c}$

Note: The blue curve is each panel is the hyperbola defined in equation (8) in the paper, and the red curve in panels (a) and (b) is the hyperbola defined in equation (9) in the paper. The intercept points $\lambda_{1}^{E X O-N I}, \lambda_{2}^{E X O-N I}, \lambda_{3}^{E X O-N I}$ and $\sigma_{1}^{E X O-N I}, \sigma_{2}^{E X O-N I}, \sigma_{3}^{E X O-N I}$ are defined as: $\lambda_{1}^{E X O-N I}=\frac{\tilde{c}-t-\tilde{p}}{v-t-\tilde{p}}$; $\lambda_{2}^{E X O-N I}=\frac{\tilde{c}-h t-\tilde{p}}{h(v-t)-\tilde{p}} ; \quad \lambda_{3}^{E X O-N I}=1-\frac{\tilde{c}}{\tilde{p}} ; \sigma_{1}^{E X O-N I}=\frac{1}{2}+\frac{h(v-\tilde{c})-(1-h) \tilde{c}}{2[h(v-\tilde{c})+(1-h) \tilde{c}]} ; \quad \sigma_{2}^{E X O-N I}=\frac{1}{2}+$ $\frac{(1-h)(\tilde{c}-\tilde{p})-h(\tilde{p}-\tilde{c}+t)}{2[h(\tilde{p}-\tilde{c}+t)+(1-h)(\tilde{c}-\tilde{p})]} ; \sigma_{3}^{E X O-N I}=\frac{1}{2}+\frac{h(\tilde{p}-\tilde{c}+t)-(1-h)(\tilde{c}-\tilde{p})}{2[h(\tilde{p}-\tilde{c}+t)+(1-h)(\tilde{c}-\tilde{p})]}$. 


\section{Appendix D. Proof of Proposition 4: provision strategy with exogenous precision and full insurance}

To analyze expert's provision behavior with exogenous precision and with full insurance, we proceed in a similar way as in Appendix C (EXO-NI case). That is, we first ignore Strategy D and derive the provision areas for the case where only strategies $\mathrm{A}, \mathrm{B}$ and $\mathrm{C}$ are available. Later we show that in area $\mathrm{X} \in\{\mathrm{A}, \mathrm{B}, \mathrm{C}\}$, Strategy X strictly dominates Strategy D. ${ }^{2}$

The boundary between strategies A and C (as specified in equation (10) in the paper) is now defined by $\sigma_{A C}^{E X O-F I}=f(\lambda, h, \tilde{p}, \tilde{c}, v, t)=\frac{h(\tilde{c}-\tilde{p})-h \lambda(v-t)-h t}{(1-2 h)(\tilde{p}-\tilde{c})-h \lambda(v-t)-h t} \quad$ or $\quad \lambda_{A C}^{E X O-F I}=f(\sigma, h, \tilde{p}, \tilde{c}, v, t)=$ $\frac{(h+\sigma-2 h \sigma)(\tilde{p}-\tilde{c})+h(1-\sigma) t}{h(1-\sigma)(t-v)}$. The boundary between strategies B and C (as specified in equation (11) in the paper) is defined by $\quad \sigma_{B C}^{E X O-F I}=f(\lambda, h, \tilde{p}, \tilde{c}, v, t)=\frac{(1-h)(\tilde{p}-\tilde{c})}{(1-2 h)(\tilde{p}-\tilde{c})-h \lambda(v-t)-h t} \quad$ or $\quad \lambda_{B C}^{E X O-F I}=$ $f(\sigma, h, \tilde{p}, \tilde{c}, v, t)=\frac{(1-h-\sigma+2 h \sigma)(\tilde{p}-\tilde{c})+h \sigma t}{h \sigma(t-v)}$.

Next, we derive the vertices of the hyperbolas $\sigma_{A C}^{E X O-N I}$ and $\sigma_{B C}^{E X O-N I}$ to define the areas in the $(\lambda, \sigma)$ space in which each of the three strategies is optimal for the expert. The vertex of hyperbola $\sigma_{A C}^{E X O-F I}$ is $V \lambda_{A C}^{E X O-F I}=\frac{(1-h)(\tilde{p}-\tilde{c})}{h(v-t)}$, while the vertex of hyperbola $\sigma_{B C}^{E X O-F I}$ is $V \lambda_{B C}^{E X O-F I}=\frac{-(1-h)(\tilde{p}-\tilde{c})}{h(v-t)}$.

As in the EXO-NI case, the two hyperbolas are geometrically similar and mirroring each other. Furthermore, the respective hyperbola does not exist when the associated vertex is 0 . The latter is true iff $\tilde{p}=\tilde{c}$. Consequently, when $\tilde{p}=\tilde{c}$, the vertex $V \lambda_{A C}^{E X O-F I}$ changes from positive to negative (i.e. the curve changes from concave to convex), while the vertex $V \lambda_{B C}^{E X O-F I}$ changes vice versa (i.e. the curve changes from convex to concave).

Next, let us derive the boundary values of the functions $\lambda_{A C}^{E X O-F I}, \sigma_{A C}^{E X O-F I}, \lambda_{B C}^{E X O-F I}$ and $\sigma_{B C}^{E X O-F I}$.

- $\lambda_{B C}^{E X O-F I}(\sigma=1)=\lambda_{1}^{E X O-F I}=\frac{\tilde{c}-t-\tilde{p}}{v-t}$ is the $\lambda$-value of the function $\lambda_{B C}^{E X O-F I}$ when $\sigma=1$.

- $\quad \lambda_{B C}^{E X O-F I}(\sigma=0.5)=\lambda_{A C}^{E X O-F I}(\sigma=0.5)=\lambda_{2}^{E X O-F I}=\frac{\tilde{c}-h t-\tilde{p}}{h(v-t)}$ is the $\lambda$-value of the function $\lambda_{B C}^{E X O-F I}$ or function $\lambda_{A C}^{E X O-F I}$ when $\sigma=0.5$.

- $\quad \lambda_{A C}^{E X O-F I}(\sigma=1)=\infty$ is the $\lambda$-value of the function $\lambda_{A C}^{E X O-F I}$ when $\sigma=1$.

- $\sigma_{A C}^{E X O-F I}(\lambda=1)=\sigma_{1}^{E X O-F I}=\frac{1}{2}+\frac{h(\tilde{c}-\tilde{p}-v)-(1-h)(\tilde{p}-\tilde{c})}{2[(1-h)(\tilde{p}-\tilde{c})+h(\tilde{c}-\tilde{p}-v)]}$ is the $\sigma$-value of the function $\sigma_{A C}^{E X O-F I}$ when $\lambda=1$.

- $\sigma_{B C}^{E X O-F I}(\lambda=0)=\sigma_{2}^{E X O-F I}=\sigma_{2}^{E X O-N I}=\frac{1}{2}+\frac{(1-h)(\tilde{c}-\tilde{p})-h(\tilde{p}-\tilde{c}+t)}{2[h(\tilde{p}-\tilde{c}+t)+(1-h)(\tilde{c}-\tilde{p})]}$ is the $\sigma$-value of the function $\sigma_{B C}^{E X O-F I}$ when $\lambda=0$.

- $\sigma_{A C}^{E X O-F I}(\lambda=0)=\sigma_{3}^{E X O-F I}=\sigma_{3}^{E X O-N I}=\frac{1}{2}+\frac{h(\tilde{p}-\tilde{c}+t)-(1-h)(\tilde{c}-\tilde{p})}{2[h(\tilde{p}-\tilde{c}+t)+(1-h)(\tilde{c}-\tilde{p})]}$ is the $\sigma$-value of the function $\sigma_{A C}^{E X O-F I}$ when $\lambda=0$.

- $\sigma_{B C}^{E X O-F I}(\lambda=1)=\sigma_{4}^{E X O-F I}=\frac{1}{2}+\frac{(1-h)(\tilde{p}-\tilde{c})-h(\tilde{c}-\tilde{p}-v)}{2[(1-h)(\tilde{p}-\tilde{c})+h(\tilde{c}-\tilde{p}-v)]}$ is the $\sigma$-value of the function $\sigma_{B C}^{E X O-F I}$ when $\lambda=1$.

\footnotetext{
${ }^{2}$ This latter proof is similar to that in Appendix $\mathrm{C}$ and is available from the authors upon request.
} 
Next, let us analyze one-by-one the locations of the just derived boundary values on the $\lambda$-axis and the $\sigma$-axis.

1. $\lambda_{1}^{E X O-F I}=\frac{\tilde{c}-t-\tilde{p}}{v-t}$ :

i. $\quad \lambda_{1}^{E X O-F I}<0$ iff $\tilde{p}>\tilde{c}-t$.

ii. $\quad \lambda_{1}^{E X O-F I} \in[0,1]$ iff $\tilde{p}<\tilde{c}-t$.

iii. $\quad \lambda_{1}^{E X O-F I}>1$ never as long as $\tilde{c}<v$.

2. $\lambda_{2}^{E X O-F I}=\frac{\tilde{c}-h t-\tilde{p}}{h(v-t)}$ :

i. $\quad \lambda_{2}^{E X O-F I}<0$ iff $\tilde{p}>\tilde{c}-h t$.

ii. $\quad \lambda_{2}^{E X O-F I} \in[0,1]$ iff $\tilde{p}<\tilde{c}-h t$.

iii. $\quad \lambda_{2}^{E X O-F I}>1$ never as long as $\tilde{c}<h v$.

3. $\sigma_{1}^{E X O-F I}=\frac{1}{2}+\frac{h(\tilde{c}-\tilde{p}-v)-(1-h)(\tilde{p}-\tilde{c})}{2[(1-h)(\tilde{p}-\tilde{c})+h(\tilde{c}-\tilde{p}-v)]}$ :

i. $\quad \sigma_{1}^{E X O-F I}<0.5$ never, given our assumption $\tilde{c}<h v$.

ii. $\quad \sigma_{1}^{E X O-F I} \in[0.5,1]$ iff $\tilde{p}<\tilde{c}$.

iii. $\quad \sigma_{1}^{E X O-F I}>1$ iff $\tilde{p} \geq \tilde{c}$.

4. $\sigma_{2}^{E X O-F I}$ is identical to $\sigma_{2}^{E X O-N I}$ and conditions are defined in Appendix C.

5. $\sigma_{3}^{E X O-F I}$ is identical to $\sigma_{3}^{E X O-N I}$ and conditions are defined in Appendix $\mathrm{C}$.

6. $\quad \sigma_{4}^{E X O-F I}=\frac{1}{2}+\frac{(1-h)(\tilde{p}-\tilde{c})-h(\tilde{c}-\tilde{p}-v)}{2[(1-h)(\tilde{p}-\tilde{c})+h(\tilde{c}-\tilde{p}-v)]}$ : given our assumption $\tilde{c}<v$ we immediately get $\sigma_{4}^{E X O-F I}>$ 1.

Next, let us consider each sub-case of Figure 4, accounting for the underlying condition $\tilde{c}<h v$, as well as for the additional condition $\tilde{c}>t$. We derive typical locations of the curves $\lambda_{A C}^{E X O-F I}$ and $\lambda_{B C}^{E X O-F I}$ when $\tilde{p}$ falls in the specific interval and compare these with the $\lambda_{A C}^{E X O-N I}$ and the $\lambda_{B C}^{E X O-N I}$ curve.

a. Panel (a) corresponds to the case where $0<\tilde{p}<\tilde{c}-t$. In this case $\lambda_{A C}^{E X O-F I}$ is concave and $\lambda_{B C}^{E X O-F I}$ is convex, since $\tilde{p}<\tilde{c}$. Both function intersect at $\lambda_{2}^{E X O-F I}$. The price difference restriction immediately yields that $\lambda_{1}^{E X O-F I} \in[0,1]$ and $\lambda_{2}^{E X O-F I} \in[0,1]$ since $\tilde{p}<\tilde{c}-t<\tilde{c}-h t ; \sigma_{1}^{E X O-F I} \in$ $[0.5,1]$. Compared to the NI case, Area $\mathrm{C}$ shifts to the left with $\lambda_{1}^{E X O-F I}<\lambda_{1}^{E X O-N I}, \lambda_{2}^{E X O-F I}<$ $\lambda_{2}^{E X O-N I}$, and $\sigma_{1}^{E X O-F I}>\sigma_{1}^{E X O-N I}$. The dynamics of the intercept points $\lambda_{1}^{E X O-F I}, \lambda_{2}^{E X O-F I}$, $\sigma_{1}^{E X O-F I}$ as $\tilde{p}$ increases from 0 to $\tilde{c}-t$ is similar to the respective intercepts of the EXO-NI case, namely $\lambda_{1}^{E X O-F I}$ and $\lambda_{2}^{E X O-F I}$ decrease and $\sigma_{1}^{E X O-F I}$ increases as $\tilde{p}$ increases.

b. Panel (b) corresponds to the case where $\tilde{c}-t<\tilde{p}<\tilde{c}-h t$. Under this price difference restriction $\lambda_{A C}^{E X O-F I}$ remains concave and $\lambda_{B C}^{E X O-F I}$ is convex, since $\tilde{p}<\tilde{c}$. Since $\tilde{p}<\tilde{c}-h t, \lambda_{2}^{E X O-N I} \in[0,1]$ and $\sigma_{1}^{E X O-N I} \in[0.5,1]$. Given the price difference restriction $\tilde{p}>\tilde{c}-t, \lambda_{1}^{E X O-F I}<0$ and $\sigma_{2}^{E X O-F I} \in[0.5,1]$. Compared to the location of the EXO-NI functions, $\lambda_{2}^{E X O-F I}<\lambda_{2}^{E X O-N I}$ and $\sigma_{1}^{E X O-F I}>\sigma_{1}^{E X O-N I}$, while $\sigma_{2}^{E X O-F I}=\sigma_{2}^{E X O-N I}$. As $\tilde{p}$ moves from the lower limit to the upper limit, $\lambda_{2}^{E X O-F I}$ decreases, $\sigma_{1}^{E X O-F I}$ increases and $\sigma_{2}^{E X O-F I}$ decreases.

c. Panel (c) corresponds to the case where $\tilde{c}-h t<\tilde{p}<\tilde{c}$. In this range of $\tilde{p}, \lambda_{A C}^{E X O-F I}$ remains concave and $\lambda_{B C}^{E X O-F I}$ remains convex, since $\tilde{p}<\tilde{c}$. Since $\tilde{p}>\tilde{c}-h t, \lambda_{2}^{E X O-F I}<0$ and the $\lambda_{B C}^{E X O-F I}$ curve does not appear in the $(\lambda \in[0,1], \sigma \in[0.5,1])$ range, same as $\lambda_{B C}^{E X O-N I}$. Since $\tilde{c}-$ $h t<\tilde{p}<\tilde{c}, \sigma_{1}^{E X O-F I} \in[0.5,1] ; \sigma_{3}^{E X O-F I} \in[0.5,1]$ always. As compared to the EXO-NI case, Area $\mathrm{C}$ shrinks upward, since $\sigma_{1}^{E X O-F I}>\sigma_{1}^{E X O-N I}$ and $\sigma_{3}^{E X O-F I}=\sigma_{3}^{E X O-N I}$. As a consequence, the range of $\{\lambda, \sigma\}$ parameter constellations under which Strategy $\mathrm{C}$ is optimal is much smaller in FI, as compared to NI. 
d. Panel (d) corresponds to the case where $\tilde{p} \geq \tilde{c}$. In this range of $\tilde{p}, \lambda_{A C}^{E X O-F I}$ turns convex and $\lambda_{B C}^{E X O-F I}$ turns concave. Since $\tilde{p} \geq \tilde{c}, \sigma_{1}^{E X O-F I}>1$ and $\sigma_{3}^{E X O-F I}>1$, which brings the $\lambda_{A C}^{E X O-F I}$ curve outside the $(\lambda \in[0,1], \sigma \in[0.5,1])$ range, while $\lambda_{A C}^{E X O-N I}$ remains in the range of interest. As a result, when $\tilde{p} \geq \tilde{c}$ there exists no $\{\lambda, \sigma\}$ parameter constellation under which Strategy $\mathrm{C}$ is optimal when there is full insurance.

Figure 4. Expert provision behavior with exogenous precision, no insurance and full insurance.

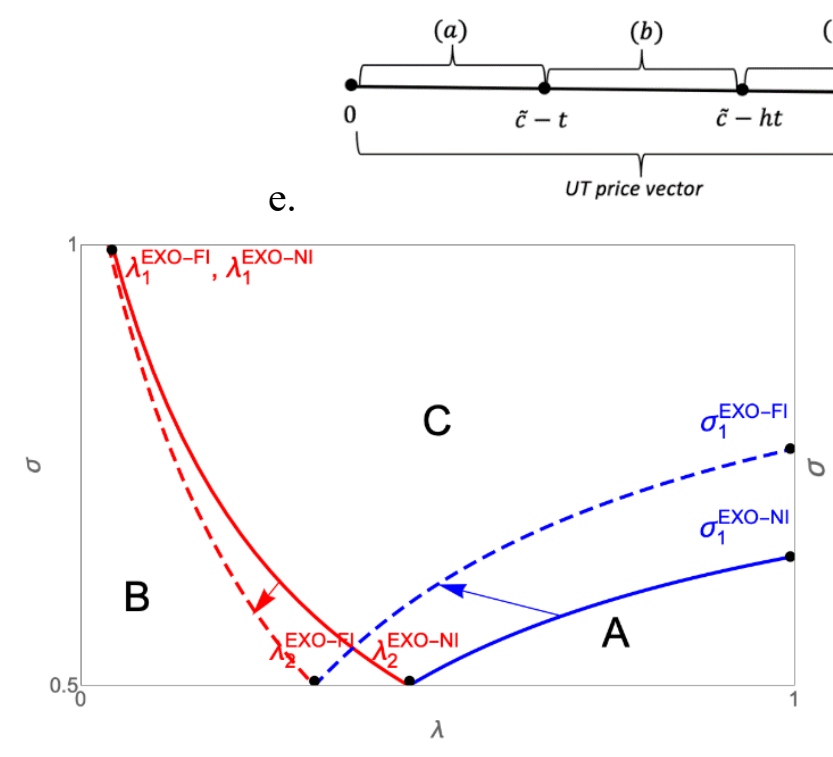

(a) $0<\tilde{p} \leq \tilde{c}-t$

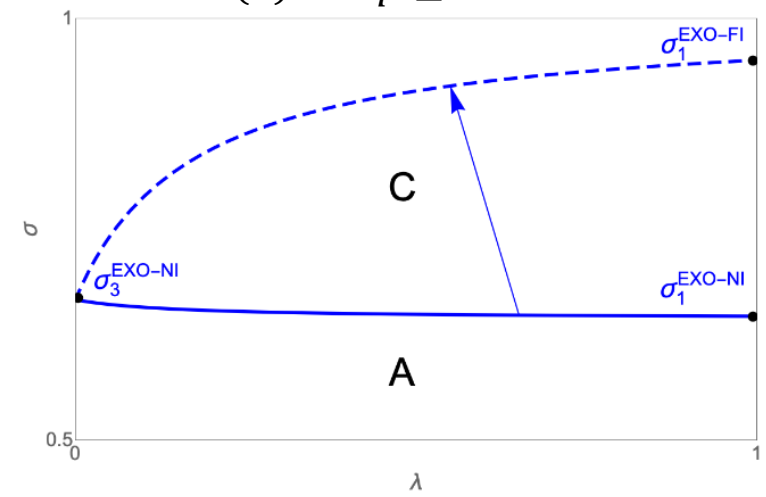

(c) $\tilde{c}-h t<\tilde{p}<\tilde{c}$ (c) $\quad(d)$

EM price vector OT price vector

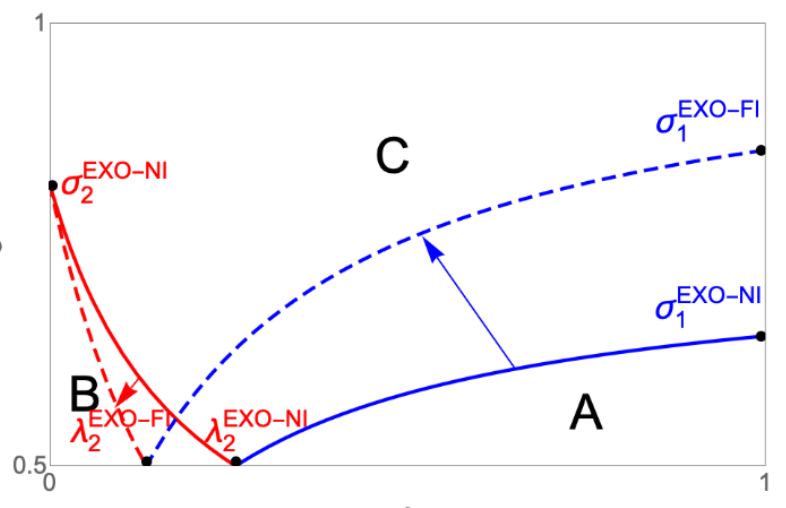

$\lambda$

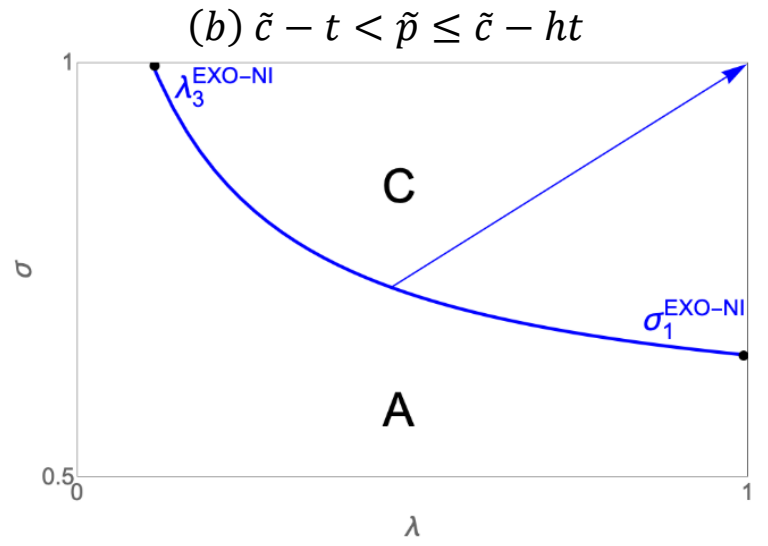

(d) $\tilde{p} \geq \tilde{c}$

Note: This figure shows the effect of introducing insurance in the setting with exogenous precision. The solid red and the solid blue line, as well as the areas A, B and C, are as defined in Proposition 3 and they characterize the optimal provision areas in the EXO-NI case. The dashed red and the dashed blue line are the hyperbolas defined in equations (10) and (11) and they define the optimal provision areas in the EXO-FI case. The intercept points $\lambda_{1}^{E X O-N I}, \lambda_{2}^{E X O-N I}, \lambda_{3}^{E X O-N I}$ and $\sigma_{1}^{E X O-N I}, \sigma_{2}^{E X O-N I}, \sigma_{3}^{E X O-N I}$ are as defined in the note to Figure 3. The intercept points $\lambda_{1}^{E X O-F I}, \lambda_{2}^{E X O-F I}$ and $\sigma_{1}^{E X O-F I}$ are defined as: $\lambda_{1}^{E X O-F I}=\frac{\tilde{c}-t-\tilde{p}}{v-t} ; \lambda_{2}^{E X O-F I}=\frac{\tilde{c}-h t-\tilde{p}}{h(v-t)} ; ; \sigma_{1}^{E X O-F I}=\frac{1}{2}+\frac{h(v-\tilde{c}+\tilde{p})-(1-h)(\tilde{c}-\tilde{p})}{2[h(v-\tilde{c}+\tilde{p})+(1-h)(\tilde{c}-\tilde{p})]^{.}}$ 


\section{Appendix E. Proof of Proposition 5: investment in information acquisition and provision behavior with no insurance}

We again start by ignoring Strategy D - verifying later that for each parameter constellation Strategy D is strictly dominated by one of the other three pure strategies. ${ }^{3}$ Strategies A, B and C are now associated with the following payoffs for the expert:

$$
\begin{aligned}
& \text { - } \quad \text { Strategy A: } \Pi_{A}=\bar{p}-\bar{c}+\lambda[v-\bar{p}] \\
& \text { - } \quad \text { Strategy B: } \Pi_{B}=\underline{p}-\underline{c}-h t+\lambda[(1-h) v-\underline{p}+h t] \\
& \text { - } \quad \text { Strategy C: } \quad \Pi_{C}=(1-h-\sigma+2 h \sigma)(\bar{p}-\bar{c})+(h+\sigma-2 h \sigma)(\underline{p}-\underline{c})- \\
& h(1-\sigma) t-d(\sigma-0.5)^{2}+\lambda[(1-h+h \sigma) v-\underline{p}(h+\sigma- \\
& 2 h \sigma)-\bar{p}(1-h-\sigma+2 h \sigma)+h(1-\sigma) t] \text {. }
\end{aligned}
$$

We derive expert investment in diagnostic precision and her provision behavior in three steps.

Step 1. We first derive the profit-maximizing diagnosis precision level assuming that investing in diagnosis and following the signal is optimal. Later we will derive the conditions under which investing in diagnosis and following the signal is optimal. To derive the optimal diagnosis effort we maximize $\Pi_{C}$ with respect to $\sigma$, which yields the profit-maximizing diagnosis precision $\sigma^{E N D O-N I}=\min \left\{\frac{1}{2}+\right.$ $\left.\frac{(1-h)(\tilde{c}-\tilde{p})-h(\tilde{c}-\tilde{p}-t)+\lambda[(1-h) \tilde{p}+h(v-t-\tilde{p})]}{2 d}, 1\right\}$, as presented in equation (12) in the body of the paper. Equation (12) implies $\sigma^{E N D O-N I} \in(0.5,1)$ for $d>(1-h)(\tilde{c}-\tilde{p})-h(\tilde{c}-\tilde{p}-t)+\lambda[(1-h) \tilde{p}+$ $h(v-t-\tilde{p})]$ and $\sigma^{E N D O-N I}=1$ for $d \leq(1-h)(\tilde{c}-\tilde{p})-h(\tilde{c}-\tilde{p}-t)+\lambda[(1-h) \tilde{p}+h(v-t-$ $\tilde{p})]$.

Step 2. We derive the boundary conditions between strategies A, B, and C and specify their geometrical properties. The boundary curves $\sigma_{A C}^{E N D O-N I}$ and $\sigma_{B C}^{E N D O-N I}$ are (quadratic) hyperbolic functions of the following (two-part) form.

- $\quad \sigma_{A C(a)}^{E N D O-N I}=\frac{\lambda((1-2 h) \tilde{p}+h(v-t))}{2 d}+$

$$
\frac{(1-2 h)(\tilde{c}-\tilde{p})+h t+d+\sqrt{d(4 h(\tilde{c}-\tilde{p}+t-\lambda(v-t-\tilde{p}))-d)+((1-2 h)(\tilde{c}-\tilde{p})+d+\lambda \tilde{p}(1-2 h)+h(\lambda(v-t)+t))^{2}}}{2 d} ;
$$

- $\quad \sigma_{A C(b)}^{E N D O-N I}=\frac{\lambda((1-2 h) \tilde{p}+h(v-t))}{2 d}-$

$$
\frac{(1-2 h)(\tilde{c}-\tilde{p})+h t+d+\sqrt{d(4 h(\tilde{c}-\tilde{p}+t-\lambda(v-t-\tilde{p}))-d)+((1-2 h)(\tilde{c}-\tilde{p})+d+\lambda \tilde{p}(1-2 h)+h(\lambda(v-t)+t))^{2}}}{2 d} ;
$$

- $\sigma_{B C(a)}^{E N D O-N I}=\frac{(1-2 h)(\tilde{c}-\tilde{p})+\lambda h(v-t)+d+h t+\sqrt{d(4(1-h)(\tilde{p}-\tilde{c})-d)+((1-2 h)(\tilde{c}-\tilde{p})+d+h(\lambda(v-t)+t))^{2}}}{2 d}$;

- $\sigma_{B C(b)}^{E N D O-N I}=\frac{(1-2 h)(\tilde{c}-\tilde{p})+\lambda h(v-t)+d+h t-\sqrt{d(4(1-h)(\tilde{p}-\tilde{c})-d)+((1-2 h)(\tilde{c}-\tilde{p})+d+h(\lambda(v-t)+t))^{2}}}{2 d}$.

Under our assumption that $\tilde{c}<h v$, only the right arm of the hyperbola $\sigma_{B C}^{E N D O-N I}$ is relevant for the range $(\lambda \in[0,1]$ and $\sigma \in[0.5,1])$. This holds true since under $\tilde{c}<h v$, hyperbola $\sigma_{B C}^{E N D O-N I}$ always

\footnotetext{
${ }^{3}$ The latter proof is similar to that in Appendix $\mathrm{C}$ and is available upon request.
} 
remains horizontal and the right arm moves leftward as $\tilde{p}$ increases. The vertex of the right arm of $\sigma_{B C}^{E N D O-N I}-\lambda_{B C}^{E N D O-N I}-$ is specified in equation (14) of the paper and it is decreasing as $\tilde{p}$ increases. The binding constraint (defined by equating $\lambda_{B C}^{E N D O-N I}$ to 0 ) is given in equation (16).

When it comes to $\sigma_{A C}^{E N D O-N I}$, depending on the magnitude of diagnosis cost $d$, either only the right arm or both arms of the hyperbola $\sigma_{A C}^{E N D O-N I}$ are relevant in the $(\lambda \in[0,1]$ and $\sigma \in[0.5,1])$ space as long as $\tilde{c}<h v$. This boundary value of $d$ is provided in equation (17) and is derived in the following way:

Unlike $\sigma_{B C}^{E N D O-N I}$, hyperbola $\sigma_{A C}^{E N D O-N I}$ turns from vertical to horizontal at some $\tilde{p}$. This $\tilde{p}$ is derived by equating the vertex of the left arm to the vertex of the right arm of $\sigma_{A C}^{E N D O-N I}$. The vertex of the right arm of $\sigma_{A C}^{E N D O-N I}-\lambda_{A C}^{E N D O-N I}$ - is specified in equation (13) in the paper. The binding constraint (defined by equating $\lambda_{A C}^{E N D O-N I}$ to 0 ) is given in equation (15). The vertex of the left arm of $\sigma_{A C}^{E N D O-N I}$ is defined as:

$\lambda_{A C}^{E N D O-N I^{*}}=\frac{d(h(v-t)-\tilde{p})-((1-2 h)(\tilde{c}-\tilde{p})+h t)}{((1-2 h) \tilde{p}+h(v-t))}+\frac{\sqrt{d\left[d(\tilde{p}-h(v-t))^{2}+4 h(1-h)((1-2 h) \tilde{p}+h(v-t)(\tilde{p} v-\widetilde{c}(v-t))]\right.}}{((1-2 h) \tilde{p}+h(v-t))^{2}}$.

By equating the two vertices and solving the resulting equality for $\tilde{p}$, we obtain the critical value of the price difference. For

$$
\tilde{p}>\tilde{p}_{A C}^{*}=\frac{h(t-v)(2(h-1)(\tilde{c}(2 h-1)+h v)+d)+2 \sqrt{(h-1)^{2} h^{2}(v-t)^{2}\left(\tilde{c}^{2}(1-2 h)^{2}+2 \tilde{c}(d+h v(1-2 h))+h v(h v-2 d)\right)}}{d+4(h-1)(2 h-1) h v},
$$

$\sigma_{A C}^{E N D O-N I}$ turns from a vertical into a conjugative horizontal hyperbola.

Step 3. The boundary conditions on $d$ are derived: For that, we insert $\tilde{p}_{A C}^{*}$ into the $\sigma_{A C}^{E N D O-N I}$ function, equate the resulting expression to 0 and solve for $\lambda$. This $\lambda$ is then equated to zero and the resulting equation is solved for $d$. This way, we obtain a boundary value for $d-d^{*}$ as defined in equation (17) of the paper - which determines whether only the right arm or both arms of $\sigma_{A C}^{E N D O-N I}$ are relevant for deriving the expert's provision behavior.

If $d<d^{*}$ (the case depicted in Figure 5), $\sigma_{A C}^{E N D O-N I}$ turns from vertical to horizontal at $\lambda<0$; thus, only the right arm appears in the $\lambda \in[0,1]$ interval.

If $d \geq d^{*}, \sigma_{A C}^{E N D O-N I}$ turns from vertical to horizontal at some $\lambda \in[0,1]$. As a result, both the left and the right arm of $\sigma_{A C}^{E N D O-N I}$ appear in the $\lambda \in[0,1]$ interval. Here we have to distinguish two subcases (see next paragraph) - they are covered in figures E1 and E2 below.

The optimal precision line $\sigma^{E N D O-N I}$ crosses the hyperbolas $\sigma_{A C}^{E N D O-N I}$ and $\sigma_{B C}^{E N D O-N I}$ at their vertices, similarly to the efficient provision case described in Appendix B. Thus, identifying the location of the vertices with respect to the $\lambda$ axis under different parameter constellations is crucial for deriving the expert's provision behavior. Since we consider $\lambda \in[0,1]$, the binding constraints (15) and (16) follow and fully characterize the location of the vertices when $d<d^{*}$. When $d \geq d^{*}$, two sub-cases need to be distinguished, depending on the location of $\lambda_{B C}^{E N D O-N I}$ at $\tilde{p}=\tilde{p}_{A C}^{*}$. Firstly, the subcase where $\lambda_{B C}^{E N D O-N I} \leq 0$ when $\tilde{p}=\tilde{p}_{A C}^{*}$; this subcase is covered in Figure E1. Secondly, the subcase where $\lambda_{B C}^{E N D O-N I}>0$ when $\tilde{p}=\tilde{p}_{A C}^{*}$; this subcase is covered in Figure E2. These two sub-cases are separated by the second boundary value of $d=d^{* * *}$. 
Due to the complex quadratic form, it is impossible to derive an explicit parametric solution for $d^{* * *}$. However, one can always obtain a numerical solution for any constellation of parameters $\{\tilde{p}, \tilde{c}, h, v, t\}$. The numerical solution can be obtained by either substituting $\tilde{p}=\tilde{p}_{A C}^{*}$ into $\lambda_{B C}^{E N D O-N I}$, setting the resulting equality to zero and solving for $d$, or by solving the following system of equations for $d$ :

$$
\left\{\begin{array}{c}
\lambda_{A C}^{E N D O-N I^{*}}=\lambda_{A C}^{E N D O-N I} \\
\lambda_{B C}^{E N D O-N I}=0 .
\end{array}\right.
$$

To better illustrate the provision strategies under endogenous diagnostic precision, we plot the hyperbolas $\sigma_{A C}^{E N D O-N I}$ and $\sigma_{B C}^{E N D O-N I}$ for each $\tilde{p}$ range considered in Figures E1 and E2. The right part of each panel depicts how the provision areas change when the endogenous choice of costly diagnosis is introduced. The left part of each panel depicts the location of the optimal precision line $\sigma^{E N D O-N I}$ and marks in which $\lambda$-range experts follow Strategy A, Strategy B, and Strategy C, respectively. Notably, the optimal precision line $\sigma^{E N D O-N I}$ always crosses the hyperbolas $\sigma_{B C}^{E N D O-N I}$ and $\sigma_{A C}^{E N D O-N I}$ at their vertex. For each $d$ range we illustrate two cases: one with relatively low $d$ and one with relatively high $d$.

Figure E1 covers the case with $d^{*} \leq d<d^{* * *}$, implying that $\lambda_{B C}^{E N D O-N I} \leq 0$ when $\tilde{p}=\tilde{p}_{A C}^{*}$. Thus, when $\tilde{p}<\tilde{p}_{B C}, \lambda=\lambda_{B C}^{E N D O-N I}$ defines the $\lambda$-range for which Strategy $\mathrm{C}$ is optimal from the left and $\lambda=1$ binds it from the right (panel (a) of Figure E1); when $\tilde{p} \in\left[\tilde{p}_{B C}, \tilde{p}_{A C}^{*}\right.$ ), $\lambda=0$ defines the $\lambda$-range for which Strategy $\mathrm{C}$ is optimal from the left and $\lambda=1$ binds it from the right (panel (b) of Figure E1), implying that all experts invest $\sigma^{E N D O-N I}$ and follow Strategy $\mathrm{C}$; when $\tilde{p} \in\left[\tilde{p}_{A C}^{*}, \tilde{p}_{A C}\right), \lambda=\lambda_{A C}^{E N D O-N I}$ defines the $\lambda$-range for which Strategy $\mathrm{C}$ is optimal from the left and $\lambda=1$ binds it from the right, while $\lambda=\lambda_{A C}^{E N D O-N I^{*}}$ binds the area in which Strategy B is optimal from the right (panel (c) of Figure E1); when $\tilde{p}>\tilde{p}_{A C}, \lambda=\lambda_{A C}^{E N D O-N I}$ defines the $\lambda$-range for which Strategy $\mathrm{C}$ is optimal from the left and $\lambda=1$ binds it from the right (panel (d) Figure E1).

Figure E2 covers the case with $d \geq d^{* * *}$, implying that $\lambda_{B C}^{E N D O-N I}>0$ when $\tilde{p}=\tilde{p}_{A C}^{*}$. The major difference compared to the previous case is that there is no parameter constellation under which all experts invest in diagnosis (like in panel (b) of Figure E1). Moreover, since $\lambda_{B C}^{E N D O-N I}>0$ when $\tilde{p}=$ $\tilde{p}_{A C}^{*}, \tilde{p}_{B C}$ is not any more the binding constrain for expert's provision behavior.

In constellations falling into panel (a) of figures E1 and E2, experts with $\lambda<\lambda_{B C}^{E N D O-N I}$ decide for Strategy B; in constellations falling into panel (c) of Figure E1 and panel (b) of Figure E2 experts with $\lambda<\lambda_{A C}^{E N D O-N I^{*}}$ decide for Strategy B and experts with $\lambda_{A C}^{E N D O-N I^{*}} \leq \lambda<\lambda_{A C}^{E N D O-N I}$ decide for Strategy $\mathrm{A}$; in constellations falling into panel (d) of Figure E1 and panel (c) of Figure E2 experts with $\lambda<$ $\lambda_{A C}^{E N D O-N I}$ decide for Strategy A. In all panels all other experts invest in diagnosis and follow the signal. Notably, under the diagnosis cost of $d \geq d^{* * *}$ there exist no parameter constellation under which all experts would invest in diagnosis. However, as long as $d^{*} \leq d<d^{* * *}$ and $\tilde{p}_{B C} \leq \tilde{p} \leq \tilde{p}_{A C}^{*}$, all experts will invest in diagnosis. 
Figure 5. Provision behavior with endogenous precision, no insurance and $\boldsymbol{d}<\boldsymbol{d}^{*}$.

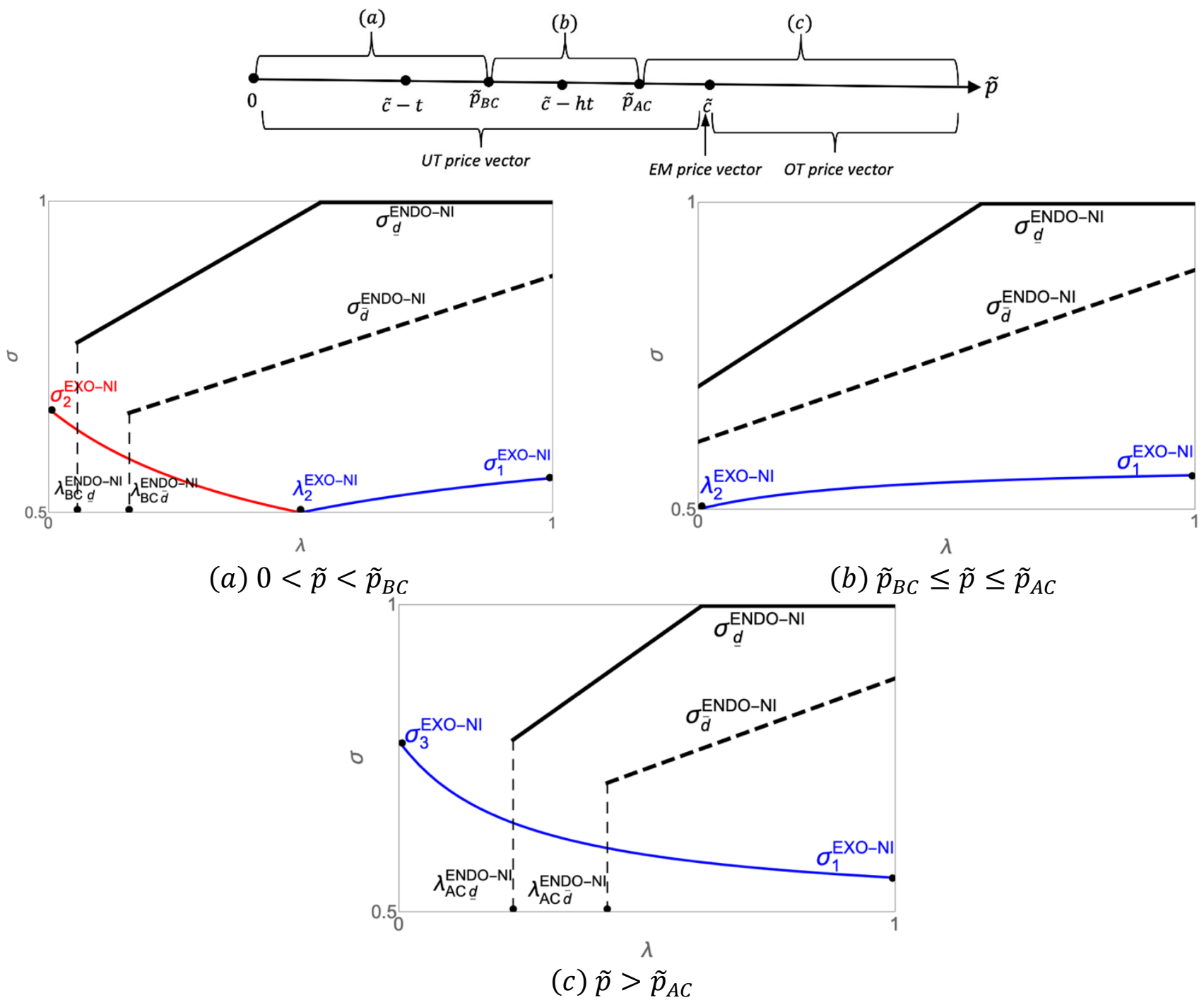

Note: This figure shows the expert's investment in information acquisition and her provision behavior in the setting with endogenous precision and no insurance for the case where $d<d^{*}-$ as defined in equation (17) in the body of the paper. The solid red and the solid blue line as well as the areas A, B and $\mathrm{C}$ are as defined in Proposition 3 and they characterize the optimal provision areas in the EXO-NI case. The solid and the dashed black line show the optimal precision in the ENDO-NI case (the solid line is valid for $d \leq(1-h) \tilde{c}+h(v-\tilde{c})$, while the dashed line covers the opposite case). The point $\lambda_{A C}^{E N D O-N I}$ is the critical $\lambda$ defined in equation (13) and the point $\lambda_{B C}^{E N D O-N I}$ is the critical $\lambda$ defined in equation (14) (in both cases, $\lambda_{x C}^{E N D O-N I}=\lambda_{x C \underline{d}}^{E N D O-N I}$ for $d \leq(1-h) \tilde{c}+h\left(v-\tilde{c}\right.$ ) and $\lambda_{x C}^{E N D O-N I}=$ $\lambda_{x C \bar{d}}^{E N D O-N I}$ for $d>(1-h) \tilde{c}+h(v-\tilde{c})$ with $\left.x \in\{A, B\}\right)$. In constellations falling into panel (a) experts with $\lambda<\lambda_{B C}^{E N D O-N I}$ decide for Strategy B and in constellations falling into panel (c) experts with $\lambda<\lambda_{A C}^{E N D O-N I}$ decide for Strategy A. In all panels all other experts invest in diagnosis and follow the signal. The optimal precision level for those experts investing in diagnosis is given by equation (12). 
Figure E1. Provision behavior with endogenous precision, no insurance and $d^{*} \leq d<d^{* * *}$.

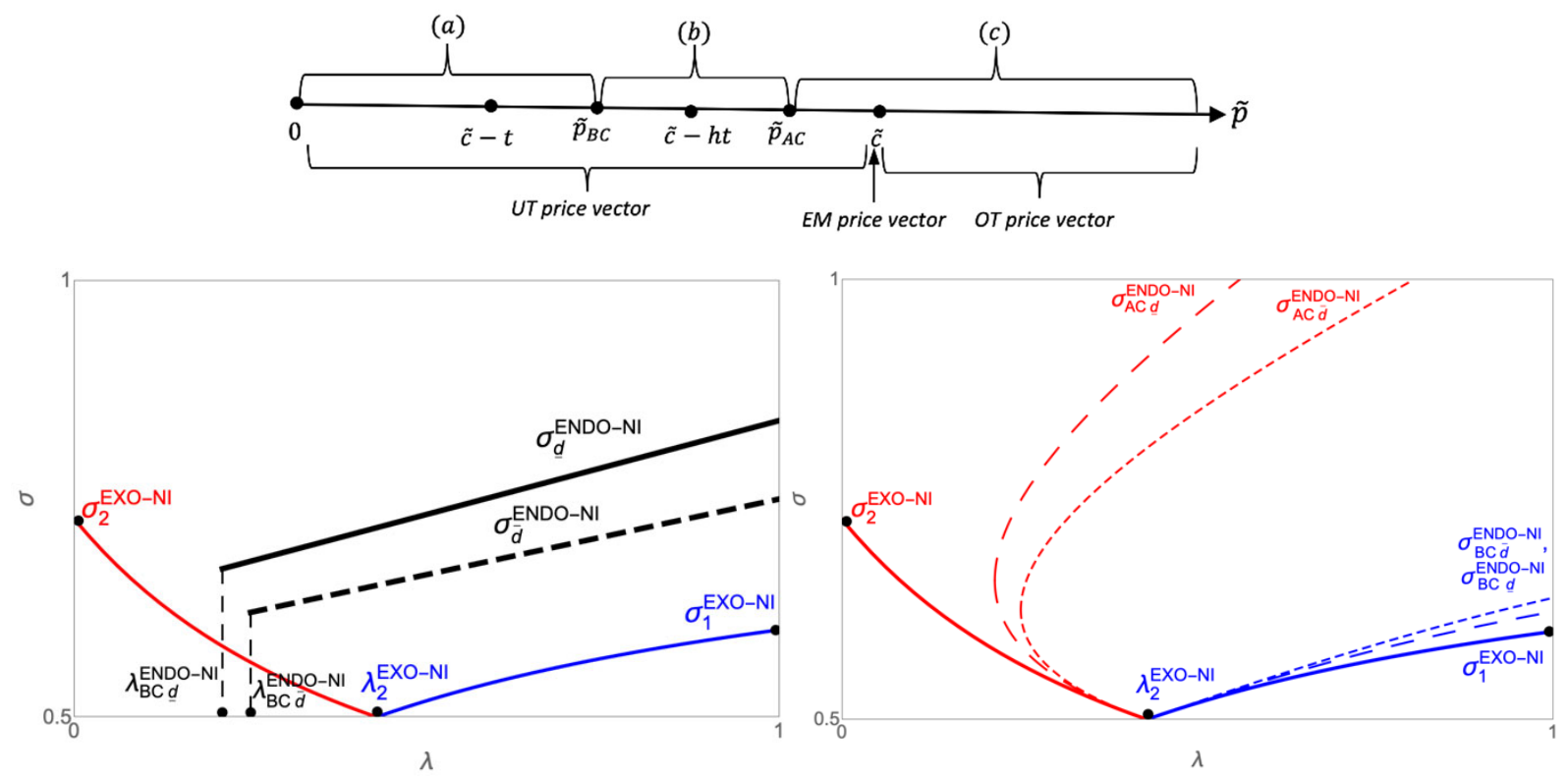

(a) $0<\tilde{p}<\tilde{p}_{B C}$

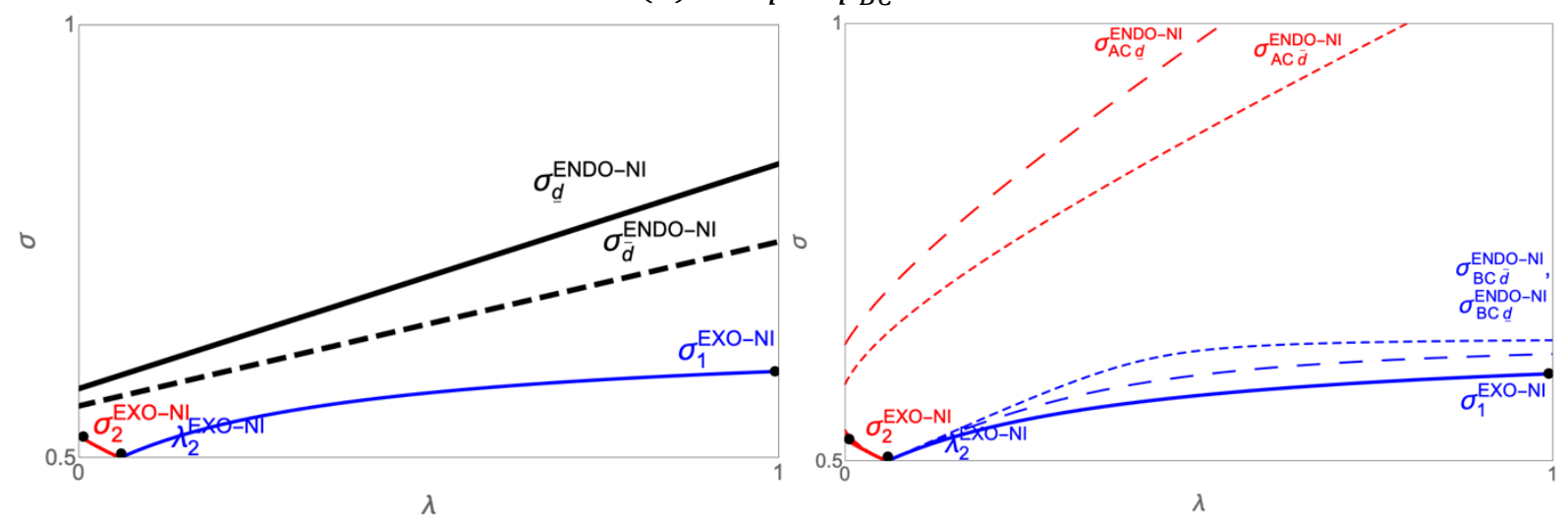

(b) $\tilde{p}_{B C} \leq \tilde{p} \leq \tilde{p}_{A C}^{*}$
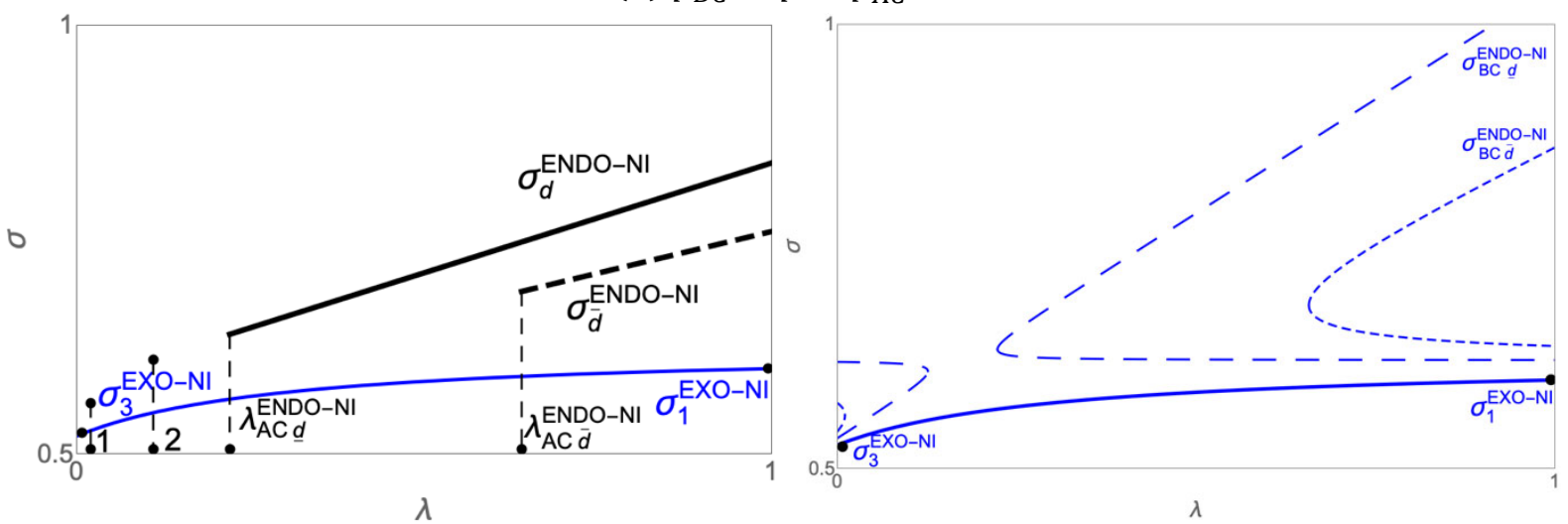

(c) $\tilde{p}_{A C}^{*}<\tilde{p} \leq \tilde{p}_{A C}$ 


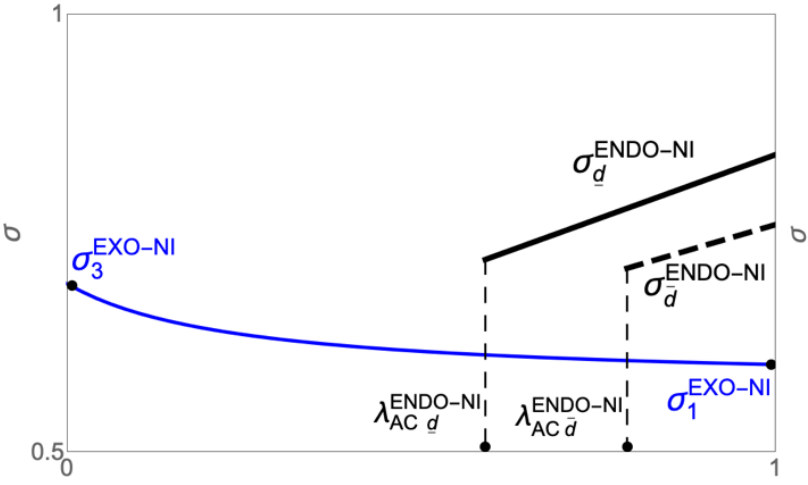

$\lambda$

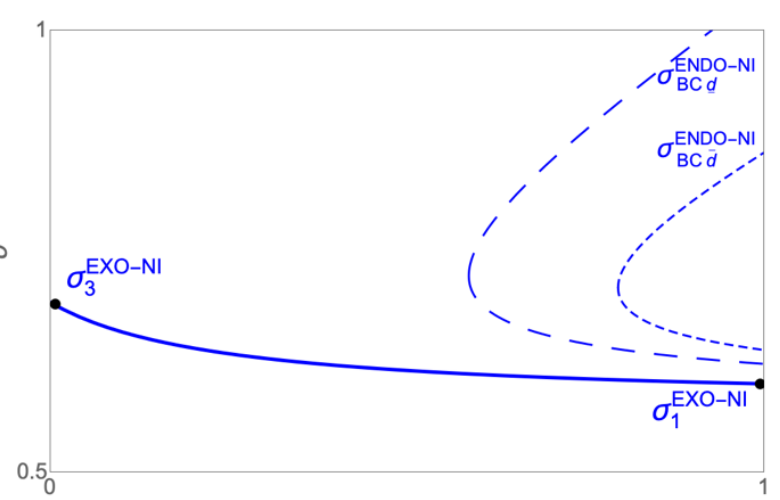

$\lambda$

(d) $\tilde{p}>\tilde{p}_{A C}$

Note: This figure shows the expert's investment in information acquisition and her provision behavior in the setting with endogenous precision and no insurance for the case where $d^{*} \leq d<d^{* * *}$. The solid red and the solid blue line as well as the areas A, B and C are as defined in Proposition 3 and they characterize the optimal provision areas in the EXO-NI case. The solid and the dashed black line show the optimal precision in the ENDO-NI case (the solid line is valid relatively low $d$ and the dashed line covers relatively high $d$, with both fulfilling $d>(1-h) \tilde{c}+h(v-\tilde{c}))$. The dashed blue curves on the right side of each panel correspond to the $\left(\sigma_{B C(a)}^{E N D O-N I}\right.$ plus $\left.\sigma_{B C(b)}^{E N D O-N I}\right)$ hyperbola, while the dashed red curves correspond to the ( $\sigma_{A C}^{E N D O-N I}$ plus $\sigma_{A C}^{E N D O-N I}$ ) hyperbola, which are defined above. The notation on the graph is as follows: Point 1 stands for $\lambda_{A C}^{E N D O-N I^{*}}$, point 2 for $\lambda_{A C}^{E N D O-N I^{*}}$. The point $\lambda_{A C}^{E N D O-N I}$ is the critical $\lambda$ defined in equation (13) and the point $\lambda_{B C}^{E N D O-N I}$ is the critical $\lambda$ defined in equation (14) (in both cases, $\lambda_{x C}^{E N D O-N I}=\lambda_{x C \underline{d}}^{E N D O-N I}$ for $d \leq(1-h) \tilde{c}+h(v-\tilde{c})$ and $\lambda_{x C}^{E N D O-N I}=\lambda_{x C \bar{d}}^{E N D O-N I}$ for $d>(1-h) \tilde{c}+h(v-\tilde{c})$ with $x \in\{A, B\})$.

Figure E2. Provision behavior with endogenous precision, no insurance, and $d \geq d^{* * *}$.

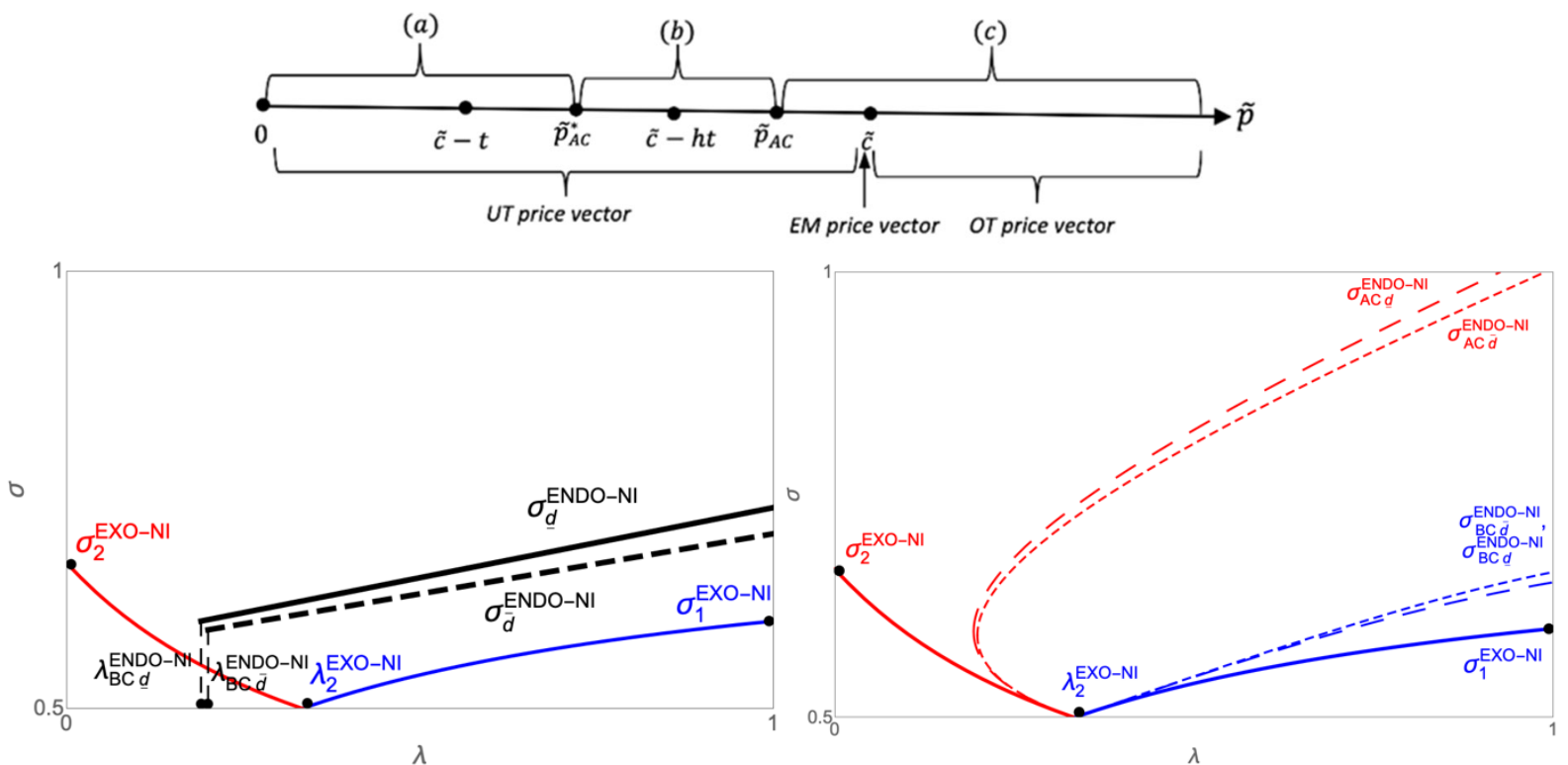

(a) $\tilde{p} \leq \tilde{p}_{A C}^{*}$ 

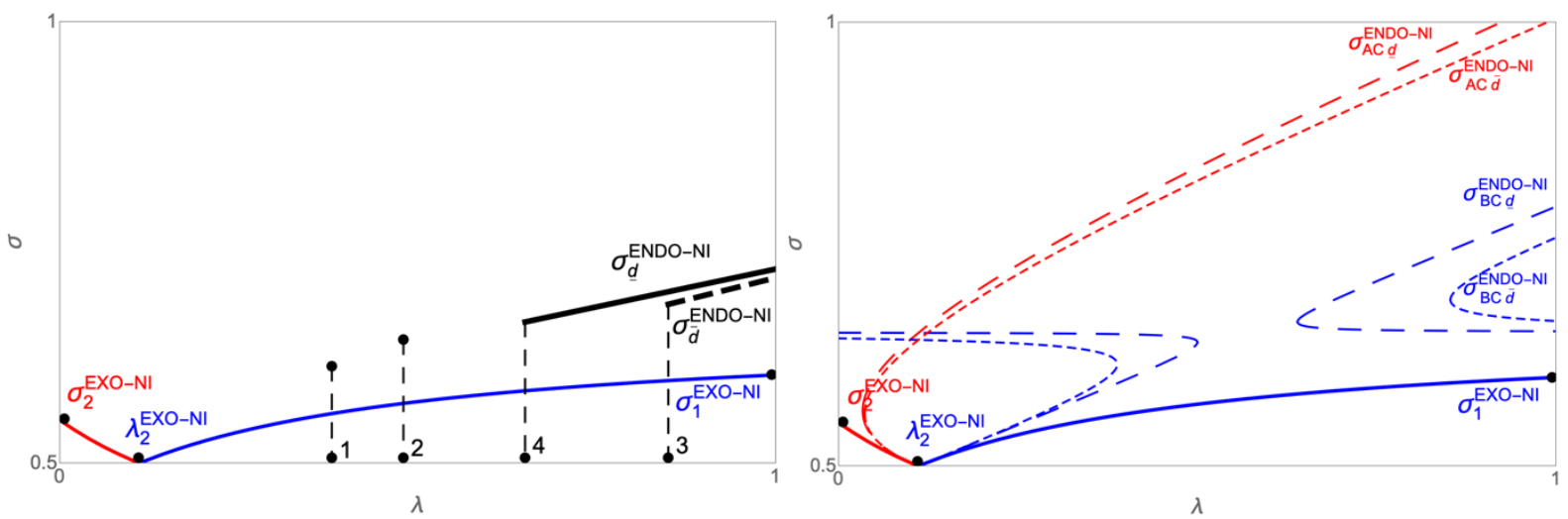

(b) $\widetilde{p}_{A C}^{*}<\tilde{p} \leq \tilde{p}_{A C}$
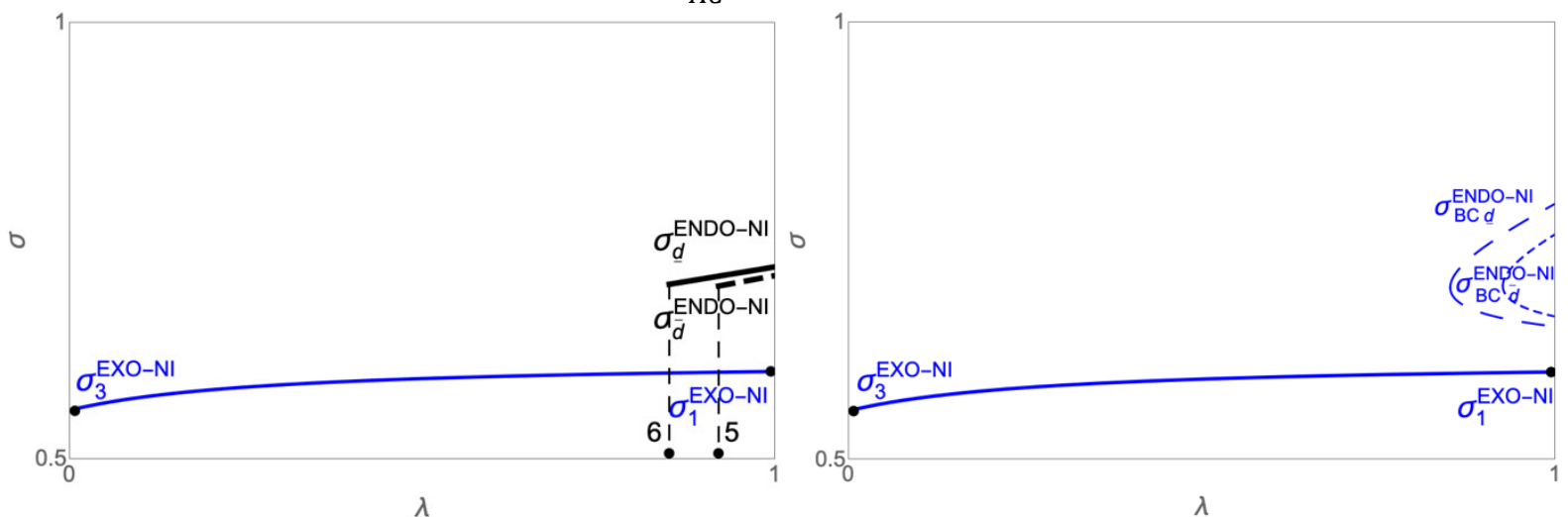

(c) $\tilde{p}>\tilde{p}_{A C}$

Note: This figure shows the expert's investment in information acquisition and her provision behavior in the setting with endogenous precision and no insurance for the case where $d \geq d^{* * *}$. The solid red and the solid blue line as well as the areas A, B and C are as defined in Proposition 3 and they characterize the optimal provision areas in the EXO-NI case. The solid and the dashed black line show the optimal precision in the ENDO-NI case (the solid line is valid for relatively low $d$ and the dashed line covers relatively high $d$, with both fulfilling $d>(1-h) \tilde{c}+h(v-\tilde{c}))$. The dashed blue curves on the right side of each panel correspond to the $\left(\sigma_{B C(a)}^{E N D O-N I}\right.$ plus $\left.\sigma_{B C(b)}^{E N D O-N I}\right)$ hyperbola, while the dashed red curves correspond to the $\left(\sigma_{A C(a)}^{E N D O-N I}\right.$ plus $\left.\sigma_{A C(b)}^{E N D O-N I}\right)$ hyperbola, which are defined above. The notation on the graph is as follows: Point 1 stands for $\lambda_{A C}^{E N D O-N I^{*}}$, point 2 for $\lambda_{A C \underline{d}}^{E N D O-N I^{*}}$, point 3 for $\lambda_{A C \bar{d}}^{E N D O-N I}$, point 4 for $\lambda_{A C \underline{d}}^{E N D O-N I}$. The point $\lambda_{A C}^{E N D O-N I}$ is the critical $\lambda$ defined in equation (13) and the point $\lambda_{B C}^{E N D O-N I}$ is the critical $\lambda$ defined in equation (14) (in both cases, $\lambda_{x C}^{E N D O-N I}=\lambda_{x C \underline{d}}^{E N D O-N I}$ for $d \leq(1-h) \tilde{c}+h(v-\tilde{c})$ and $\lambda_{x C}^{E N D O-N I}=\lambda_{x C \bar{d}}^{E N D O-N I}$ for $d>(1-h) \tilde{c}+h(v-\tilde{c})$ with $\left.x \in\{A, B\}\right)$. 


\section{Appendix F. Proof of Proposition 6: investment in information acquisition and provision behavior with insurance}

To characterize expert's provision behavior under full insurance, we proceed as in the ENDO-NI case, but consider that the consumer does not bear the cost of treatment. Strategies A, B and C are now associated with the following payoffs for the expert:

$$
\begin{array}{lll}
- & \text { Strategy A: } & \Pi_{A}=\bar{p}-\bar{c}+\lambda[v] \\
\text { - } & \text { Strategy B: } & \Pi_{B}=\underline{p}-\underline{c}-h t+\lambda[(1-h) v+h t] \\
\text { - } & \text { Strategy C: } & \Pi_{C}=(1-h-\sigma+2 h \sigma)(\bar{p}-\bar{c})+(h+\sigma-2 h \sigma)(\underline{p}-\underline{c})- \\
& h(1-\sigma) t-d(\sigma-0.5)^{2}+\lambda[(1-h+h \sigma) v+h(1-\sigma) t] .
\end{array}
$$

Similarly, as in the ENDO-NI case, we derive the expert's optimal diagnostic precision and provision strategy in three steps.

Step 1. We first derive the profit-maximizing diagnosis precision level, assuming that investing in diagnosis and following the signal is optimal. Later we will derive the conditions under which investing in diagnosis and following the signal is optimal. To derive the optimal diagnosis effort, we again maximize $\Pi_{C}$ with respect to $\sigma$, which yields the profit-maximizing diagnosis precision $\sigma^{E N D O-F I}=$ $\min \left\{\frac{1}{2}+\frac{(1-2 h)(\tilde{c}-\tilde{p})+\lambda h(v-t)+h t}{2 d}, 1\right\}$, as presented in equation (18) in the paper. It is easy to see that the optimal precision $\sigma^{E N D O-F I} \in(0.5,1)$ for $d>(1-2 h)(\tilde{c}-\tilde{p})+\lambda h(v-t)$ and $\sigma^{E N D O-F I}=1$ for $d \leq(1-2 h)(\tilde{c}-\tilde{p})+\lambda h(v-t)$.

Step 2. We derive the boundary conditions between strategies A, B, and C and specify their geometrical properties. As in the NI case, the boundary curves $\sigma_{A C}^{E N D O-F I}$ and $\sigma_{B C}^{E N D O-F I}$ are (quadratic) hyperbolic functions of the following (two-part) form.

- $\sigma_{A C(a)}^{E N D O-F I}=\frac{(1-2 h)(\tilde{c}-\tilde{p})+\lambda h(v-t)+d+h t+\sqrt{d(4 h(\tilde{c}-\tilde{p}-t-\lambda(v-t))-d)+((1-2 h) \tilde{c}+d-\tilde{p}+h(2 \tilde{p}+\lambda(v-t)+t))^{2}}}{2 d}$

- $\sigma_{A C(b)}^{E N D O-F I}=\frac{(1-2 h)(\tilde{c}-\tilde{p})+\lambda h(v-t)+d+h t-\sqrt{d(4 h(\tilde{c}-\tilde{p}-t-\lambda(v-t))-d)+((1-2 h) \tilde{c}+d-\tilde{p}+h(2 \tilde{p}+\lambda(v-t)+t))^{2}}}{2 d}$

- $\sigma_{B C(a)}^{E N D O-F I}=\frac{(1-2 h) \tilde{c}+\lambda h(v-t)+d+h t+\sqrt{d(4(1-h)(\tilde{p}-\tilde{c})-d)+((1-2 h) \tilde{c}+d-\tilde{p}+h(2 \tilde{p}+\lambda(v-t)+t))^{2}}}{2 d}$

- $\sigma_{B C(b)}^{E N D O-F I}=\frac{(1-2 h) \tilde{c}+\lambda h(v-t)+d+h t-\sqrt{d(4(1-h)(\tilde{p}-\tilde{c})-d)+((1-2 h) \tilde{c}+d-\tilde{p}+h(2 \tilde{p}+\lambda(v-t)+t))^{2}}}{2 d}$.

Under our assumption that $\tilde{c}<h v$, only the right arm of the hyperbola $\sigma_{B C}^{E N D O-F I}$ and the left arm of the hyperbola $\sigma_{A C}^{E N D O-F I}$ are relevant for the $(\lambda \in[0,1]$ and $\sigma \in[0.5,1])$ space. The vertex of the right arm of $\sigma_{B C}^{E N D O-F I}-\lambda_{B C}^{E N D O-F I}-$ is provided in equation (20) in the body of the paper, while the vertex of the left arm of $\sigma_{A C}^{E N D O-F I}-\lambda_{A C}^{E N D O-F I}-$ is provided in equation (19). $\lambda_{A C}^{E N D O-F I}$ and $\lambda_{B C}^{E N D O-F I}$ are both monotonically decreasing in $\tilde{p}$ and the binding constraints (defined by equating $\lambda_{A C}^{E N D O-F I}$ and $\lambda_{B C}^{E N D O-F I}$ to 0 ) are given in equations (15) and (16), respectively.

The boundary function $\sigma_{B C}^{E N D O-F I}$ remains horizontal as long as $\tilde{c}<h v$ under any $\tilde{p}>0$, thus, it shapes the $\lambda$-range for which Strategy $C$ is optimal as long as $\tilde{p}$ fulfils condition (16). However, condition (15) comes into force only when the hyperbola $\sigma_{A C}^{E N D O-F I}$ changes from vertical to conjugative horizontal and, technically, it happens when the vertex of the left and the right arm of $\sigma_{A C}^{E N D O-F I}$ converge. The 
formula of the left arm of $\sigma_{A C}^{E N D O-F I}$ is given by equation (19), while the vertex of the left arm of $\sigma_{A C}^{E N D O-F I}$ is defined as:

$$
\lambda_{A C}^{E N D O-F I^{*}}=\frac{(1-h)(\tilde{p}-\tilde{c})+h(\tilde{c}-\tilde{p}-t)+d}{h(v-t)}+\frac{\sqrt{d h^{2}(v-t)^{2}(d+4(h(3 \tilde{c}-\tilde{p})-(\tilde{c}-\tilde{p}))}}{h^{2}(v-t)^{2}} .
$$

By equating the two vertices and solving the resulting equality for $\tilde{p}$, we obtain the critical value of the price difference $\tilde{p}=\tilde{p}_{1}=\tilde{c}-\frac{d}{4(1-h)}$, provided in equation (21) in the paper. Thus, when $\tilde{p}=\tilde{p}_{1}$, $\sigma_{A C}^{E N D O-F I}$ turns from a vertical into a conjugative horizontal hyperbola.

Step 3. The boundary condition on $d$ is derived: To identify the $\lambda$-ranges for which each of the three strategies is optimal for a given price difference $\tilde{p}$, we distinguish two sub-cases. The first sub-case corresponds to the setting in which $\lambda_{B C}^{E N D O-F I}$ passes 0 before $\sigma_{A C}^{E N D O-F I}$ turns from a horizontal to a vertical hyperbola. The second sub-case covers the opposite case, where $\lambda_{B C}^{E N D O-F I}$ passes 0 after $\sigma_{A C}^{E N D O-F I}$ turns from a horizontal to a vertical hyperbola. These two sub-cases are distinguished by the critical value of $d=d^{* *}$, which is provided in equation (22) and obtained by solving the following system of equations with respect to $\tilde{p}$ :

$$
\left\{\begin{array}{c}
\lambda_{A C}^{E N D O-F I^{*}}=\lambda_{A C}^{E N D O-F I} \\
\lambda_{B C}^{E N D O-F I}=0
\end{array}\right.
$$

The first sub-case, corresponding to $d>d^{* *}$, is depicted in Figure 6. Under this constraint, the binding price difference constraints are $\tilde{p}_{1}<\tilde{p}_{B C}<\tilde{p}_{A C}$. As a result, when $\tilde{p}<\tilde{p}_{1}, \lambda=\lambda_{B C}^{E N D O-F I}$ defines the $\lambda$-range for which Strategy $C$ is optimal from the left and $\lambda=1$ binds it from the right (panel (a) of Figure 7); when $\tilde{p} \in\left[\tilde{p}_{1}, \tilde{p}_{B C}\right), \lambda=\lambda_{B C}^{E N D O-F I}$ defines the $\lambda$-range for which Strategy $\mathrm{C}$ is optimal from the left and $\lambda=\lambda_{A C}^{E N D O-F I}$ binds it from the right (panel (b) of Figure 7); when $\tilde{p} \in\left[\tilde{p}_{B C}, \tilde{p}_{A C}\right.$ ), $\lambda=$ 0 defines the $\lambda$-range for which Strategy $\mathrm{C}$ is optimal from the left and $\lambda=\lambda_{A C}^{E N D O-F I}$ binds it from the right (panel (c) of Figure 7); when $\tilde{p} \geq \tilde{p}_{A C}$, no expert follows Strategy C (panel (d) of Figure 7).

The second sub-case, corresponding to $d<d^{* *}$, is depicted on Figure F1. In this setting, $\tilde{p}_{B C}<\tilde{p}_{1}<$ $\tilde{p}_{A C}$. The resulting $\lambda$-ranges in which Strategy $\mathrm{C}$ is optimal under different $\tilde{p}$ intervals are quite similar to the $d>d^{* *}$ case, with only some minor differences: When $\tilde{p}<\tilde{p}_{B C}$ under $d \leq d^{* *}$, the provision areas are shaped similarly as in the $\tilde{p}<\tilde{p}_{1}$ case under $d>d^{* *}$ (thus, panels (a) of Figures 7 and F1 are comparable); when $\tilde{p} \in\left[\tilde{p}_{1}, \tilde{p}_{B C}\right), \lambda=0$ defines the $\lambda$-range for which Strategy $\mathrm{C}$ is optimal from the left and $\lambda=1$ binds it from the right (panel (b) of Figure F1), implying that all experts invest $\sigma^{E N D O-F I}$ and follow the signal; when $\tilde{p} \in\left[\tilde{p}_{B C}, \tilde{p}_{A C}\right), \lambda=0$ defines the $\lambda$-range in which Strategy $\mathrm{C}$ is optimal from the left and $\lambda=\lambda_{A C}^{E N D O-F I}$ binds it from the right (panel (c) of Figures 7 and F1); when $\tilde{p} \geq \tilde{p}_{A C}$, no expert follows Strategy C (panel (d) of Figures 7 and F1). 
Figure 6. Provision behavior with endogenous precision, full insurance, and $\boldsymbol{d}<\boldsymbol{d}^{* *}$.

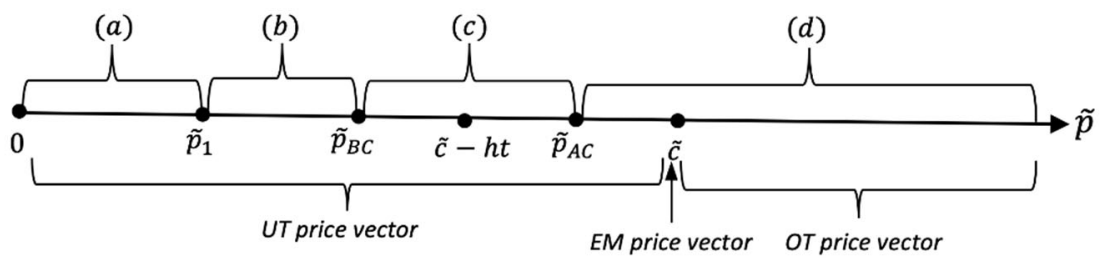

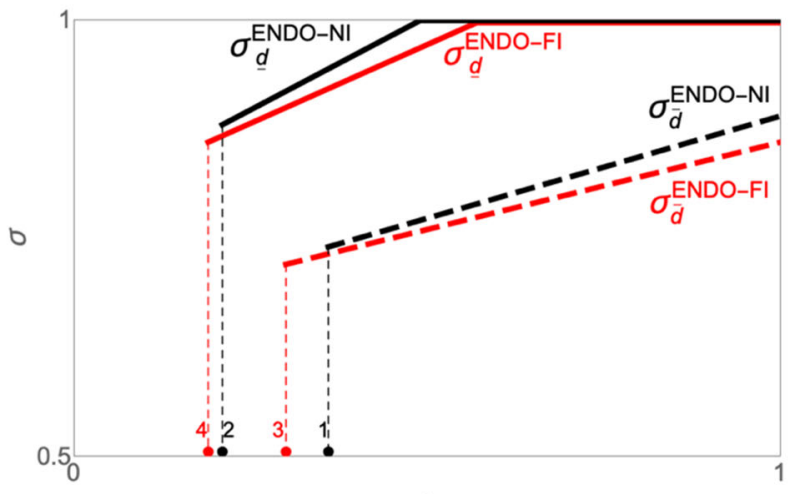

$\lambda$

(a) $0<\tilde{p} \leq \tilde{p}_{1}$

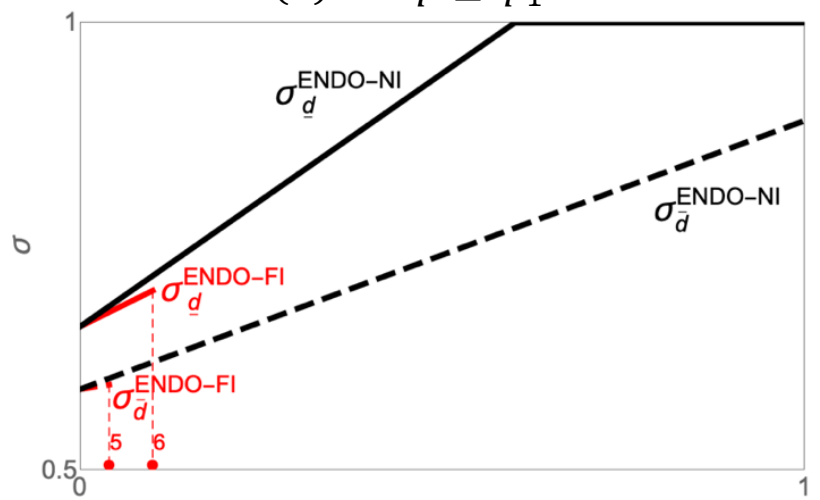

$\lambda$

(c) $\tilde{p}_{B C} \leq \tilde{p} \leq \tilde{p}_{A C}$

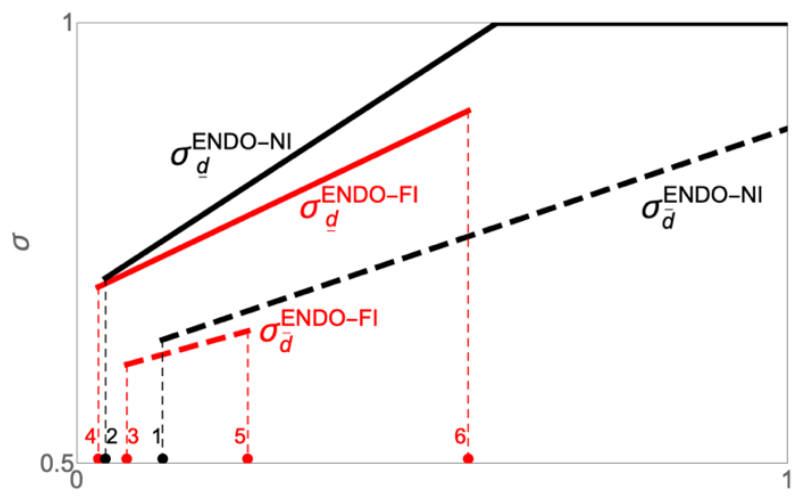

$\lambda$

(b) $\tilde{p}_{1}<\tilde{p}<\tilde{p}_{B C}$

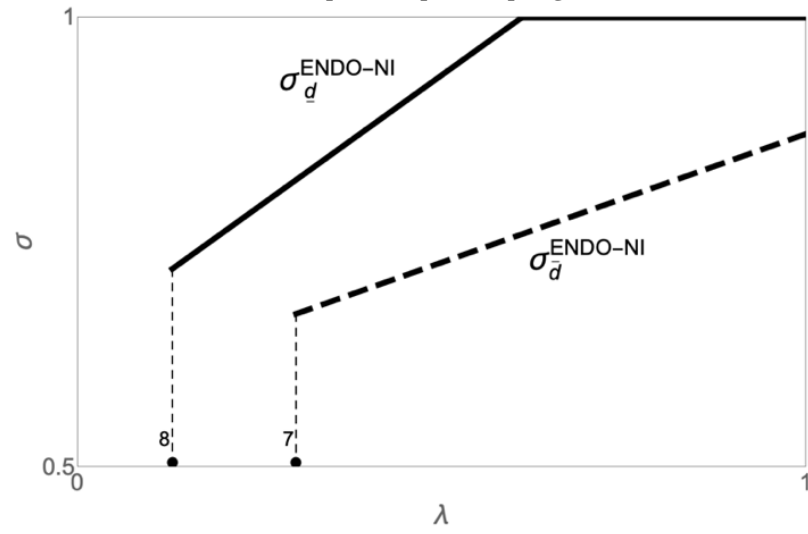

(d) $\tilde{p}>\tilde{p}_{A C}$

Note: The figure shows the effect of introducing insurance on the expert's investment in information acquisition and her provision behavior in the setting with endogenous precision for the case where $d<$ $d^{* *}$ - as defined in equation (22) in the body of the paper. The solid and the dashed black line are as defined in Proposition 5 and they show the optimal precision in the ENDO-NI case. The solid and the dashed red line in panels (a), (b), (c) and (d) show the optimal precision in the ENDO-FI case. For $\tilde{p} \geq$ $\tilde{p}_{A C}$ no expert invests in information acquisition and all experts decide for Strategy A - which explains why there is no red line in panel (d). The notation on the graph is as follows: Point 1 stands for $\lambda_{B C}^{E N D O-N I}$, point 2 for $\lambda_{B C d}^{E N D O-N I}$, point 3 for $\lambda_{B C \bar{d}}^{E N D O-F I}$, point 4 for $\lambda_{B C{ }^{d} d}^{E N D O-F I}$, point 5 for $\lambda_{A C \bar{d}}^{E N D O-F I}$, point 6 for $\lambda_{A C \underline{d}}^{E N D O-F I}$, point 7 for $\lambda_{A C \bar{d}}^{E N D O-N I}$, and point 8 for $\lambda_{A C \underline{d}}^{E N D O-N I}$. The point $\lambda_{A C}^{E N D O-F I}$ is the critical $\lambda$ defined in equation (19) and the point $\lambda_{B C}^{E N D O-F I}$ is the critical $\lambda$ defined in equation (20) (in both cases, $\lambda_{x C}^{E N D O-F I}$ $=\lambda_{x C \underline{d}}^{E N D O-F I}$ for $d \leq(1-h) \tilde{c}+h(v-\tilde{c})$ and $\lambda_{x C}^{E N D O-F I}=\lambda_{x C \bar{d}}^{E N D O-F I}$ for $d>(1-h) \tilde{c}+h(v-\tilde{c})$ with $x \in\{A, B\}$ ). In constellations falling into panels (a) and (b) experts with $\lambda<\lambda_{B C}^{E N D O-F I}$ decide for Strategy B and in constellations falling into panels (b) and (c) experts with $\lambda>\lambda_{A C}^{E N D O-F I}$ decide for Strategy A. In panels (a), (b) and (c) all other experts invest in diagnosis and follow the signal, while in 
panel (d) all experts decide for Strategy A. The optimal precision level for those experts investing in diagnosis is given by equation (18).

Figure F1. Provision behavior with endogenous precision, full insurance, and $d \geq d^{* *}$.

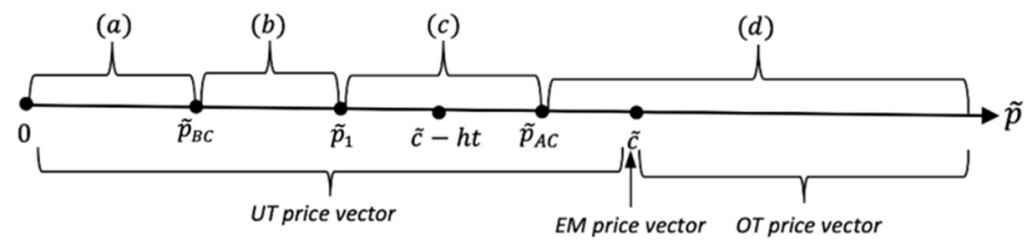

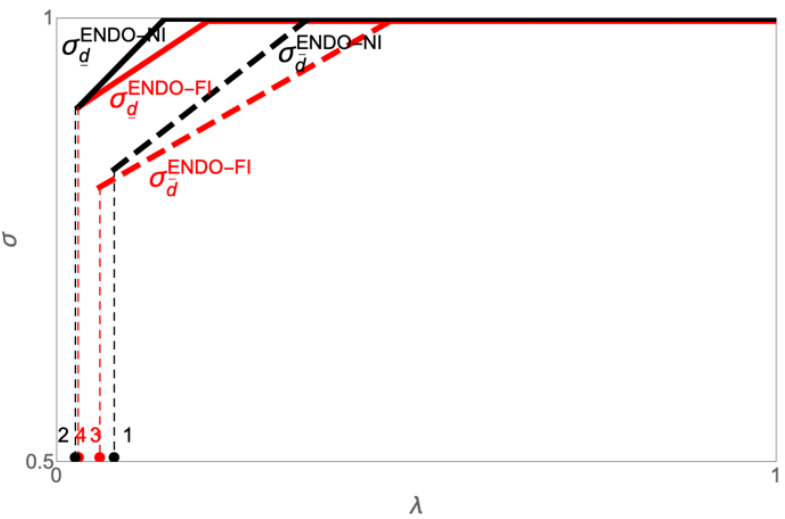

(a) $0<\tilde{p} \leq \tilde{p}_{B C}$

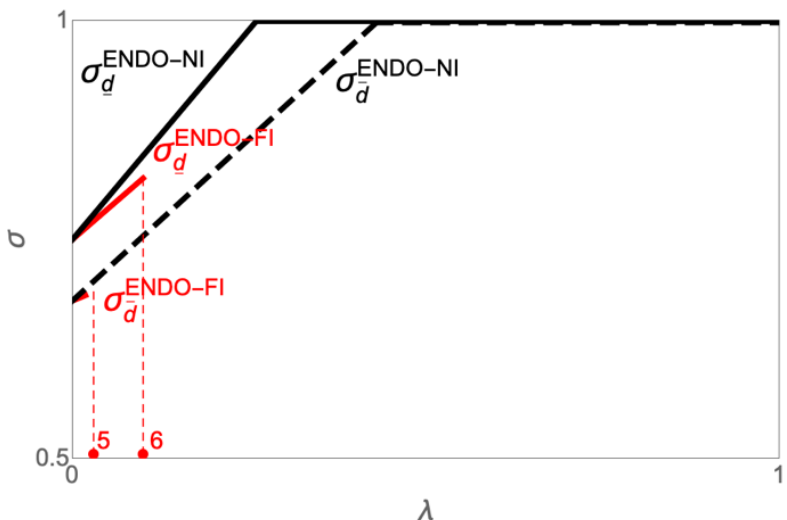

(c) $\tilde{p}_{1} \leq \tilde{p} \leq \tilde{p}_{A C}$

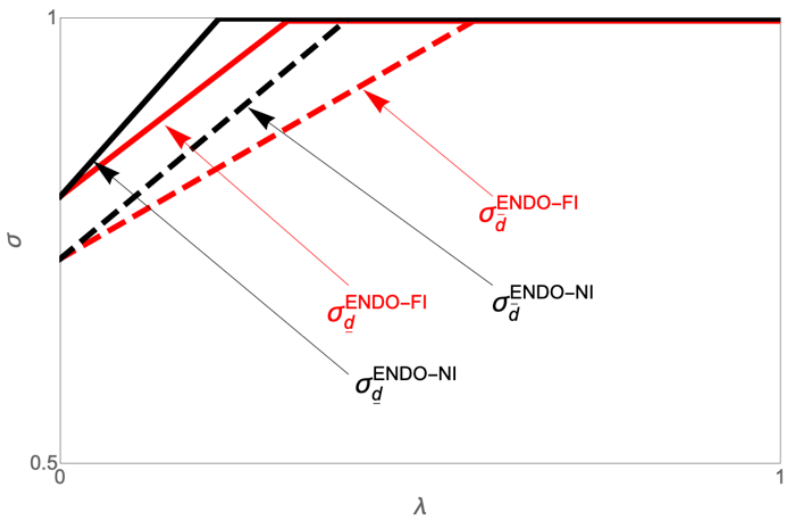

(b) $\tilde{p}_{B C}<\tilde{p}<\tilde{p}_{1}$

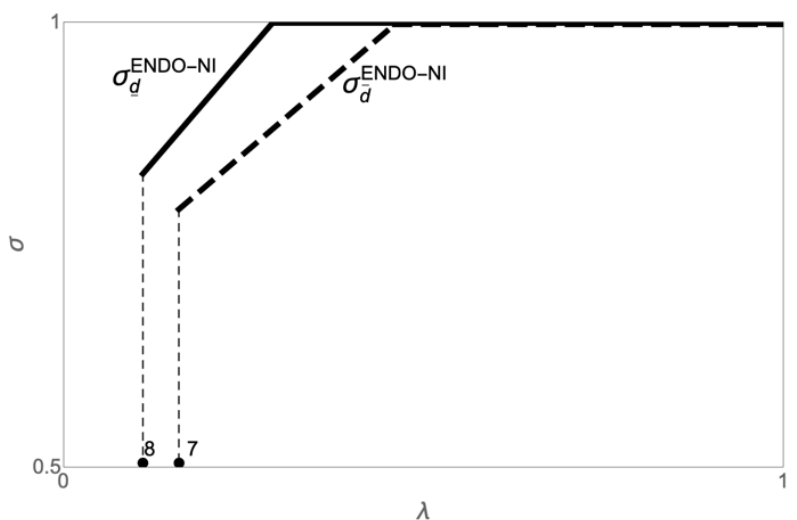

(d) $\tilde{p}>\tilde{p}_{A C}$

Note: The figure shows the effect of introducing insurance on the expert's investment in information acquisition and her provision behavior in the setting with endogenous precision for the case where $d \geq$ $d^{* *}-$ as defined in equation (22) in the body of the paper. The explanation for the lines and the notation are the same as in Figure 6 (see note to Figure 6). In constellations falling into panel (a) experts with $\lambda<\lambda_{B C}^{E N D O-F I}$ decide for Strategy B and in constellations falling into panels (c) experts with $\lambda>$ $\lambda_{A C}^{E N D O-F I}$ decide for Strategy A. In panels (a), (b) and (c) all other experts invest in diagnosis and follow the signal, while in panel (d) all experts decide for Strategy A. The optimal precision level for those experts investing in diagnosis is given by equation (18). 
Appendix G. Parameters of the EET and translation of EET results in parameter values

\section{Parameters in the Equality Equivalence Test}

Table G1. Parameters in the Equality Equivalence Test.

Advantageous Inequality Block

\begin{tabular}{cc|cc}
\hline \multicolumn{2}{c|}{ LEFT } & \multicolumn{2}{c}{ RIGHT } \\
\hline $\begin{array}{c}\text { decision maker's } \\
\text { payoff }\end{array}$ & $\begin{array}{c}\text { passive person's } \\
\text { payoff }\end{array}$ & $\begin{array}{c}\text { decision maker's } \\
\text { payoff }\end{array}$ & $\begin{array}{c}\text { passive person's } \\
\text { payoff }\end{array}$ \\
\hline $8 \mathrm{ECU}$ & $7 \mathrm{ECU}$ & $10 \mathrm{ECU}$ & $10 \mathrm{ECU}$ \\
\hline $9 \mathrm{ECU}$ & $7 \mathrm{ECU}$ & $10 \mathrm{ECU}$ & $10 \mathrm{ECU}$ \\
\hline $10 \mathrm{ECU}$ & $7 \mathrm{ECU}$ & $10 \mathrm{ECU}$ & $10 \mathrm{ECU}$ \\
\hline $11 \mathrm{ECU}$ & $7 \mathrm{ECU}$ & $10 \mathrm{ECU}$ & $10 \mathrm{ECU}$ \\
\hline $12 \mathrm{ECU}$ & $7 \mathrm{ECU}$ & $10 \mathrm{ECU}$ & $10 \mathrm{ECU}$ \\
\hline
\end{tabular}

Disadvantageous Inequality Block

\begin{tabular}{cc|cc}
\hline \multicolumn{2}{c|}{ LEFT } & \multicolumn{2}{c}{ RIGHT } \\
\hline $\begin{array}{c}\text { decision maker's } \\
\text { payoff }\end{array}$ & $\begin{array}{c}\text { passive person's } \\
\text { payoff }\end{array}$ & $\begin{array}{c}\text { decision maker's } \\
\text { payoff }\end{array}$ & $\begin{array}{c}\text { passive person's } \\
\text { payoff }\end{array}$ \\
\hline $8 \mathrm{ECU}$ & $13 \mathrm{ECU}$ & $10 \mathrm{ECU}$ & $10 \mathrm{ECU}$ \\
\hline $9 \mathrm{ECU}$ & $13 \mathrm{ECU}$ & $10 \mathrm{ECU}$ & $10 \mathrm{ECU}$ \\
\hline $10 \mathrm{ECU}$ & $13 \mathrm{ECU}$ & $10 \mathrm{ECU}$ & $10 \mathrm{ECU}$ \\
\hline $11 \mathrm{ECU}$ & $13 \mathrm{ECU}$ & $10 \mathrm{ECU}$ & $10 \mathrm{ECU}$ \\
\hline $12 \mathrm{ECU}$ & $13 \mathrm{ECU}$ & $10 \mathrm{ECU}$ & $10 \mathrm{ECU}$ \\
\hline
\end{tabular}

Note: Decision makers had to indicate, for each of the ten decision situations, whether they wanted to implement the LEFT or the RIGHT allocation 
Denote the own material payoff by $m$ and the other's payoff by $o$. Suppose subjects in the lab decide in line with the utility function $u(m, o)=m+\lambda o$, as assumed for experts in our model. What can we infer about $\lambda$ from their choices in the EET?

Advantageous Inequality Block (Y-List)

\begin{tabular}{|c|c|c|}
\hline $\begin{array}{c}\text { subject chooses LEFT for } \\
\text { the first time in row }\end{array}$ & inference regarding $\lambda_{\mathbf{A}}$ & attributed $\lambda_{\mathbf{A}}$ \\
\hline 1 (always left) & $\lambda_{\mathrm{A}} \leq-2 / 3$ & $\lambda_{\mathrm{A}}=-5 / 6$ \\
\hline 2 & $-2 / 3 \leq \lambda_{\mathrm{A}} \leq-1 / 3$ & $\lambda_{\mathrm{A}}=-3 / 6$ \\
\hline 3 & $-1 / 3 \leq \lambda_{\mathrm{A}} \leq 0$ & $\lambda_{\mathrm{A}}=-1 / 6$ \\
\hline 4 & $0 \leq \lambda_{\mathrm{A}} \leq 1 / 3$ & $\lambda_{\mathrm{A}}=1 / 6$ \\
\hline 5 & $1 / 3 \leq \lambda_{\mathrm{A}} \leq 2 / 3$ & $\lambda_{\mathrm{A}}=3 / 6$ \\
\hline never (always right) & $2 / 3 \leq \lambda_{\mathrm{A}}$ & $\lambda_{\mathrm{A}}=5 / 6$ \\
\hline
\end{tabular}

Disadvantageous Inequality Block (X-List)

\begin{tabular}{|c|c|c|}
\hline $\begin{array}{c}\text { subject chooses LEFT for } \\
\text { the first time in row }\end{array}$ & inference regarding $\lambda_{\mathbf{D}}$ & attributed $\lambda_{\mathbf{D}}$ \\
\hline 1 (always left) & $2 / 3 \leq \lambda_{\mathrm{D}}$ & $\lambda_{\mathrm{D}}=5 / 6$ \\
\hline 2 & $1 / 3 \leq \lambda_{\mathrm{D}} \leq 2 / 3$ & $\lambda_{\mathrm{D}}=3 / 6$ \\
\hline 3 & $0 \leq \lambda_{\mathrm{D}} \leq 1 / 3$ & $\lambda_{\mathrm{D}}=1 / 6$ \\
\hline 4 & $-1 / 3 \leq \lambda_{\mathrm{D}} \leq 0$ & $\lambda_{\mathrm{D}}=-1 / 6$ \\
\hline 5 & $-2 / 3 \leq \lambda_{\mathrm{D}} \leq-1 / 3$ & $\lambda_{\mathrm{D}}=-3 / 6$ \\
\hline never (always right) & $\lambda_{\mathrm{D}} \leq-2 / 3$ & $\lambda_{\mathrm{D}}=-5 / 6$ \\
\hline
\end{tabular}




\section{Diagnostic Uncertainty and Insurance Coverage in Credence Goods Markets}

Loukas Balafoutas, Helena Fornwagner, Rudolf Kerschbamer, Matthias Sutter and Maryna Tverdostup

\section{Supplementary Appendix Experimental Part}

\section{Table of Contents:}

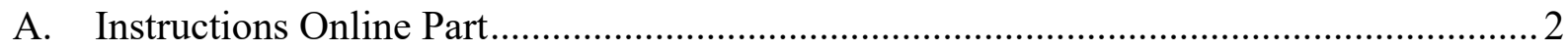

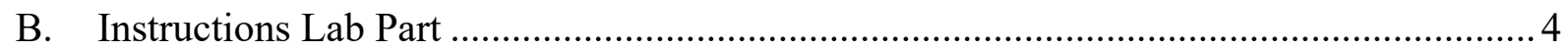

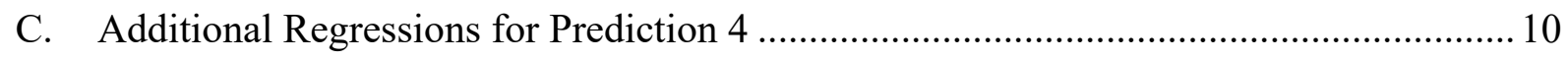


This following sections contain the instructions translated from German, which were used in the online and lab part of the experiment. We read out all instructions out loud in the lab. If instructions were different between treatments, this is indicated by squared brackets, including the respective treatment condition and the according text in italic letters. To match the observations from the online and the lab part, participants created a unique in the online part and had to state this ID at the beginning of the lab part.

\section{A. Instructions Online Part}

\section{Instructions}

The first part of the experiment will be conducted online and consists of 10 decisions. In each of these 10 decisions, the computer randomly assigns another participant to you. In the following, we will call this participant "Your Passive Person". You will never learn the identity of your passive person. You will see below why we call this person a "passive person".

We state the payoffs in ECU (experimental currency units).

The exchange rate is $5 \mathrm{ECU}=1$ Euro

Each of your 10 choices is a choice between the LEFT and the RIGHT option. Each option has consequences for your payoff and the payoff of your passive person.

Example:

You will be asked if you prefer to choose the option LEFT, where you will receive 8 ECU and your passive person 13 ECU, or option RIGHT, where you will receive 10 ECU. Your passive person will also receive 10 ECU. You have to choose one of the two options by clicking on the corresponding circle.

This decision problem would be presented on the screen as follows: 


\section{Your decision}

Option 'Left'

Option 'Right'

\begin{tabular}{|c|c|c|c|c|c|}
\hline Your Payoff & $\begin{array}{c}\text { Payoff passive per- } \\
\text { son }\end{array}$ & & & Your Payoff & Payoff passive person \\
\hline $8,00 \mathrm{ECU}$ & $13,00 \mathrm{ECU}$ & $\mathrm{O}$ & $\mathrm{O}$ & $10,00 \mathrm{ECU}$ & $10,00 \mathrm{ECU}$ \\
\hline
\end{tabular}

You have to make a total of 10 decisions ( 5 are shown per screen). Your total earnings from this part are determined as follows:

Payout as Active Person: In the end, one out of the 10 decision-making situations is selected individually and randomly for each participant, and the option chosen in this decision situation is then actually paid out. E.g., if you have chosen the decision situation described above and you have decided on the option RIGHT in this decision situation, you would receive 10 ECU as an active person. In contrast, your passive person would receive $10 \mathrm{ECU}$ as a passive person.

Payout as Passive Person: Just as your Passive Person receives ECU from your decision without doing anything, you receive ECU from another participant without doing anything. This means you are the passive person for this other participant. It is assured that you will not be redeemed twice as an active and passive person. That is, if person $\mathrm{X}$ is your passive person, then you are certainly not the passive person of person $\mathrm{X}$.

You will see 5 of all 10 decisions on one screen. You can make corrections to your choices as long as you have not clicked "Next".

After the 10 decisions, this part of the experiment ends. Which of your 10 decisions is relevant for your payoff and how much you have earned, you will get to know in the EconLab, when you participate in the lab part. Also, this online part will be paid at the EconLab in cash. 


\section{B. Instructions Lab Part}

\section{$\underline{\text { Instructions }}$}

Thank you for your participation in the experiment. Please do not talk to other participants until the end of the experiment.

In this experiment, the payoffs are stated in ECU (experimental currency units).

The exchange rate is $80 \mathrm{ECU}=1 €$.

\section{Different roles:}

There are two roles in the experiment: client and expert. At the beginning of the experiment, you will be randomly assigned one of these roles and keep that role for the entire experiment, which means for all periods. At the first decision screen (period 1 of 24), you will see your role.

In each period, one expert is randomly assigned to one client (and vice versa). It is ensured in each period that the same client-expert pair is never formed in two consecutive periods. This means that a client always interacts with an expert, and you will randomly get a partner in each period.

\section{$\underline{24 \text { Periods }}$}

This experiment consists of 24 periods, each with the same sequence of decisions described below.

Suppose you have been assigned a client's role. In that case, you have a big problem with a probability of $40 \%$ and a small problem with a probability of $60 \%$. As a client, you will never be informed about the problem you actually have. As a client, you can decide if you enter the market, which means you want to interact with the expert randomly assigned to you. By entering the market, your problem may be solved by the expert. If your problem is solved, you will receive $150 \mathrm{ECU}$ in this period. If your problem is not solved, the expert has to pay you a compensation of $50 \mathrm{ECU}$ (more on this below).

[EXO100]

- If you have been assigned the role of an expert, you will receive in each period a signal about what problem your client (randomly matched with you) has. This signal is 100\% 
accurate. That is, if e.g., the client has a small problem, then, with 100\% probability, you get the signal that the problem is small; and similar to the big problem. However, this information costs 10 ECU. These costs will be deducted automatically in each period.

[EXO70]

- If you have been assigned the role of an expert, you will receive in each period a signal about what problem your client (randomly matched with you) has. This signal is $70 \%$ accurate. If e.g., the client has a small problem, then, with 70\% probability, you get the signal that the problem is small; and similar to the big problem. However, this information costs 1.6 ECU. These costs will be deducted automatically in each period.

\section{[ENDO]}

If you have been assigned an expert's role, you will receive a signal each period about the client's problem randomly. As an expert, you decide on the precision of the received signal. The costs associated with each precision level are given in the following table:

$$
\begin{aligned}
& \text { Precision of the signal } \\
& 50 \% \quad 0 E C U \\
& 60 \% \quad 0.4 E C U \\
& 70 \% \quad 1.6 \mathrm{ECU} \\
& 80 \% \quad 3.6 \mathrm{ECU} \\
& 90 \% \quad 6.4 \mathrm{ECU} \\
& 100 \% \quad 10 \mathrm{ECU}
\end{aligned}
$$

For example, suppose you choose a 70\% precise signal. In that case, you pay 1.6 ECU and get a signal that identifies the problem correctly with a $70 \%$ probability. If you select a $50 \%$ precision, you will not be charged. However, your signal is not informative (because it identifies the actual problem with the same probability as right or wrong). 
As an expert, your job is to treat the client. You can choose between a low quality treatment and a high quality treatment. The low quality treatment only solves the small problem of the client. The high quality treatment solves both the small and the big problem. You always have to pay ECU 20 for a low quality treatment and $60 \mathrm{ECU}$ for a high quality treatment. If your treatment has not solved the client's problem, then as an expert, you have to pay a compensation fee of 50 ECU.

For the treatment of low quality, you will receive from the client a price of 60 ECU. For high quality treatment, the price is either $80 \mathrm{ECU}, 100 \mathrm{ECU}$, or $120 \mathrm{ECU}$ : this price varies over periods, and you will find out the respective price at the beginning of each period.

- Treatment can only be carried out if the client has decided to enter the market. If the client chooses not to enter the market, that is, if he /she decides not to interact with the expert, both client and expert receive a payoff of $15 \mathrm{ECU}$ in this period.

\section{Overview of the Decisions of one Period}

Therefore, each period consists of decisions of the expert and the client, which are made simultaneously and independently of each other.

\begin{tabular}{|c|c|}
\hline $\begin{array}{l}\text { Client: } \\
\text { The client decides if he/she wants to enter } \\
\text { the market. }\end{array}$ & $\begin{array}{l}\text { Expert: } \\
\text { [ENDO] } \\
\text { 1. The expert decides on the signal preci- } \\
\text { sion. } \\
\text { 2. The expert receives the signal about the } \\
\text { client's problem and decides if he/she wants } \\
\text { to use the high or low quality treatment. } \\
\text { [EXO100] and [EXO70] } \\
\text { The expert receives the signal about the cli- } \\
\text { ent's problem and decides whether he /she } \\
\text { wants to implement the high or low quality } \\
\text { treatment. }\end{array}$ \\
\hline
\end{tabular}


Only after both made their decisions it is announced whether the client has entered the market or not. If the client has not entered, the expert's decisions are irrelevant. In summary, this leads to the following payoffs:

\section{$\underline{\text { Payoffs }}$}

CLIENT DOES NOT ENTER THE MARKET: both the client and the expert receive 15 ECU.

\section{CLIENT ENTERS THE MARKET:}

\section{Client:}

If the problem was solved: $\quad 150 \mathrm{ECU}$ minus the treatment price

If the problem was NOT solved: $\quad 50 \mathrm{ECU}$ minus the treatment price

\section{Expert:}

[EXO70] If the problem was solved:

treatment price minus treatment cost minus 1,6

(costs diagnostic precision 70\%)

[EXO100] If the problem was solved:

treatment price minus treatment cost minus 10 (costs diagnostic precision 100\%)

[EXO70] If the problem was not solved: treatment price minus treatment cost minus 50 ECU (compensation) minus 1,6 (costs diagnostic precision 70\%)

[EXO100] If the problem was not solved: treatment price minus treatment cost minus 50 ECU (compensation) minus 10 (costs diagnostic precision 100\%)

\section{[ENDO]}

If the problem was solved: treatment price minus treatment cost minus the cost for the selected signal precision level

If the problem was not solved: treatment price minus treatment cost minus 50 ECU (compensation) minus cost for the selected signal precision level 


\section{Insurance}

Please note that there are two possible market situations in each period: either the client is insured or not. You play a total of 12 periods with and 12 periods without insurance, and you will see at the beginning of each period on the screen if the particular period is a period with insurance or not.

In a period without insurance, the situation is the same as previously described. In a period with insurance, the client is insured. This means that at the beginning of such a period, the client pays an insurance premium of $80 \mathrm{ECU}$ (deducted automatically, even if the client does not enter the market). In return, the price for the client's treatment is covered by the insurance. All decisions taken by the client and the expert remain the same as in periods without insurance. The payoffs of the two parties in periods with insurance are as follows:

Payoffs in periods with insurance:

Client:

CLIENT DOES NOT ENTER THE MARKET: 15 ECU minus 80 ECU (insurance premium) $=-65 \mathrm{ECU}$

\section{CLIENT ENTERS THE MARKET:}

If the problem has been solved: $150 \mathrm{ECU}$ minus $80 \mathrm{ECU}$ (insurance premium) $=70 \mathrm{ECU}$ If the problem was NOT solved: $50 \mathrm{ECU}$ minus $80 \mathrm{ECU}$ (insurance premium) $=-30 \mathrm{ECU}$ Expert:

For the expert, the payoff does not change. It is calculated as in periods without insurance.

\section{Information and Feedback}

In each period, the expert and the client get to know whether the client is insured in this period and the prices for the low and high quality. The expert also receives the signal about the problem of the client. After the second period, you can see the results of all past periods at the bottom of your screen. 


\section{Total Payoff}

At the beginning of the experiment, you will receive an endowment of 80 ECU. From this endowment, you can also cover possible losses in individual periods. Profits from other periods also compensate for losses.

For the final payoff, each period's endowment and the payoffs are added together and paid in cash at the end of the experiment, using the exchange rate $80 \mathrm{ECU}=1 €$. Also, you will receive your payoff from the online part. 


\section{Additional Regressions for Prediction 4}

Table C.1 Determinants of efficient Service Provision by Insurance Condition

\begin{tabular}{lcc}
\hline & $\mathrm{NI}$ & $\mathrm{FI}$ \\
\hline EXO70 & $-0.07^{* * *}$ & $-0.06^{* *}$ \\
UT price vector & $(0.02)$ & $(0.02)$ \\
& $0.09^{* * *}$ & $0.14^{* * *}$ \\
OT price vector & $(0.02)$ & $(0.02)$ \\
& $-0.08^{* * *}$ & -0.03 \\
Period & $(0.02)$ & $(0.02)$ \\
& -0.00 & $-0.00^{* *}$ \\
Risk measure & $(0.00)$ & $(0.00)$ \\
& -0.00 & $-0.02^{* * *}$ \\
$\lambda_{D}$ & $(0.01)$ & $(0.01)$ \\
& 0.04 & 0.03 \\
$\lambda_{A}$ & $(0.03)$ & $(0.04)$ \\
\multirow{2}{*}{ Female } & $0.07^{* * *}$ & -0.01 \\
& $(0.03)$ & $(0.02)$ \\
Age & -0.01 & -0.00 \\
& $(0.03)$ & $(0.03)$ \\
$N$ & -0.00 & -0.00 \\
N & $(0.00)$ & $(0.00)$ \\
\hline
\end{tabular}

Notes: Dependent variable is Follow, equal to 1 if the expert followed the received signal and 0 otherwise. The table presents marginal effects estimates from Probit regressions with subject random effects. Standard errors are clustered at the matching group level and stated in parentheses. EXO70 is equal to 1 for the EXO70 treatment. UT price vector is equal to 1 if the price for the LQS is 60 and the price for the HQS is 80 . OT price vector is equal to 1 if the price for the LQS is 60 and the price for the HQS is 120. The omitted benchmark is the EM vector with the price of 60 for LQS and of 100 for HQS. The Risk Measure and the prosociality parameters $\lambda_{\mathrm{D}}$ and $\lambda_{\mathrm{A}}$ are defined as in the notes to Table $4 .{ }^{*} \mathrm{p}<0.10,{ }^{* *} \mathrm{p}<0.05,{ }^{* * *} \mathrm{p}<0.01$. 